\title{
Changes in U.S. Family Finances from 2004 to 2007: Evidence from the Survey of Consumer Finances
} (Errata paragraph added on March 6, 2009; see p. A56)

Brian K. Bucks, Arthur B. Kennickell, Traci L. Mach, and Kevin B. Moore, of the Board's Division of Research and Statistics, prepared this article with assistance from Gerhard Fries, Daniel J. Grodzicki, and Richard A. Windle.

The Federal Reserve Board's Survey of Consumer Finances for 2007 provides insights into changes in family income and net worth since the 2004 survey. ${ }^{1}$ The survey shows that, over the 2004-07 period, the median value of real (inflation-adjusted) family income before taxes was little changed; median income had grown slightly in the preceding three-year period (figure 1). Across most demographic groups, the pattern of change was mixed, but a few changes stand out: Income increased markedly for Hispanic or nonwhite families, while it declined substantially for families living in the Northeast or the Midwest and for families headed by a person who was retired or otherwise not working. In contrast to median income, mean income in the recent period climbed 8.5 percent, and the increases were spread broadly across demographic groups. The increases were most striking for families in the top 10 percent of the distribution of net worth and for families headed by a single parent, a person who was self-employed, or a person who was aged 65 to 74 . Over the preceding three years, mean income had declined broadly. Differences in the rates of change in the median and mean signal a change in the distribution of income.

Unlike family income over the 2004-07 period, both median and mean net worth increased; the median rose 17.7 percent, and the mean rose 13.0 percent (figure 2). The increases were fairly broadly spread, but with a number of noteworthy exceptions, some of which entailed changes in medians and

1. For a detailed discussion of the 2001 and 2004 surveys as well as references to earlier surveys, see Brian K. Bucks, Arthur B. Kennickell, and Kevin B. Moore (2006), "Recent Changes in U.S. Family Finances: Evidence from the 2001 and 2004 Survey of Consumer Finances," Federal Reserve Bulletin, vol. 92, pp. A1-A38, www.federalreserve.gov/pubs/bulletin/default.htm.
1. Change in median and mean incomes, 1998-2007 SCF

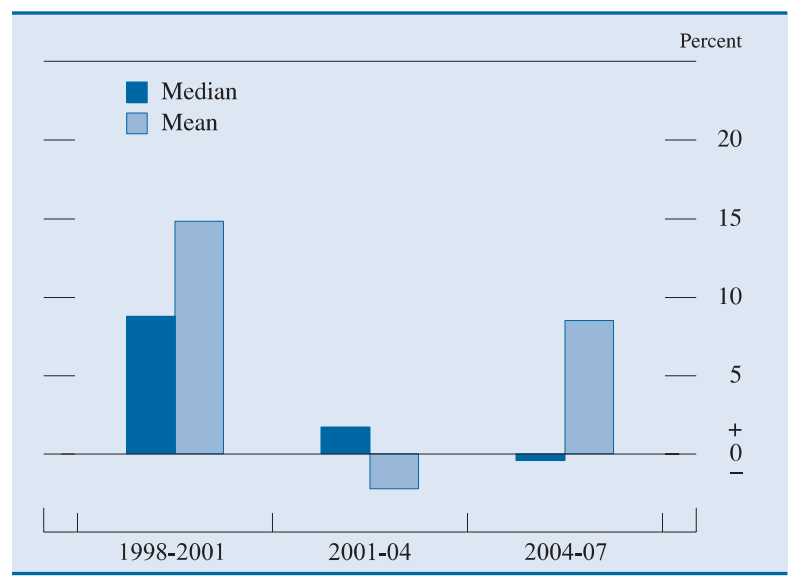

SourCE: Federal Reserve Board, Survey of Consumer Finances.

means within demographic groups that differed substantially, either in terms of relative magnitude or in the direction of change. Median and mean net worth for the lowest 25 percent of the distribution of net worth plunged 36.8 percent and 43.8 percent, respectively; median net worth for the lowest 20 percent of the distribution of income fell 1.2 percent, but the mean rose 31.8 percent. Percentage increases in

2. Change in median and mean net worth, 1998-2007 SCF

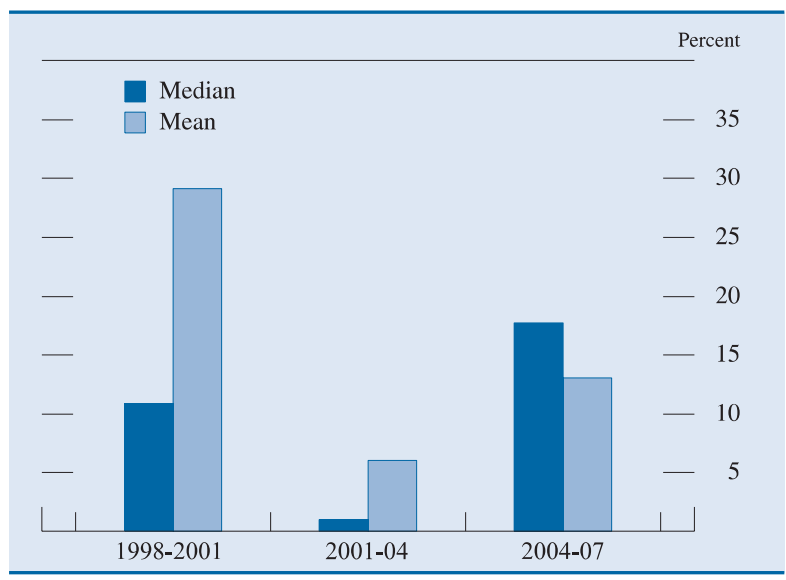

SouRCE: Federal Reserve Board, Survey of Consumer Finances. 
median and mean net worth were similar for white non-Hispanic families, while the increase in the median for nonwhite or Hispanic families was only about one-fifth of that for other families, and the increase in the mean was nearly three times the size of that for other families. Relative to other regions, both the Northeast and the Midwest saw sizable declines in median net worth. The clearest gains in both median and mean net worth were for high-networth families, high-income families, families headed by a person aged 65 or older, and families headed by a person who worked for someone else or who worked in a technical, sales, or service occupation. In the preceding three years, median net worth had increased only slightly ( 1.0 percent), while the mean had risen more strongly ( 6.0 percent); over that time, the data had shown a more complex pattern of mixed increases and decreases in wealth.

Unrealized capital gains were a particularly important factor in the increase in net worth over the 2004-07 period. The share of total assets attributable to unrealized capital gains from real estate, businesses, stocks, or mutual funds rose 5.1 percentage points, to 35.8 percent in 2007. Although the level of debt owed by families rose noticeably, debt as a percentage of assets was little changed. The largest percentage change in debt was in borrowing for residential real estate other than a primary residence.

With median and mean debt advancing faster than income, payments relative to income might be expected to increase substantially. In fact, total payments relative to total income barely increased, and the median of payments relative to income rose at a slower pace than it did between 2001 and 2004. Nonetheless, the share of families with high payments relative to their incomes increased notably.

This article reviews these and other changes in the financial condition of U.S. families between 2004 and 2007. ${ }^{2}$ The discussion draws on data from the Federal Reserve Board's Survey of Consumer Finances (SCF) for those years; it also uses evidence from earlier years of the survey to place the 2004-07 changes in a broader context.

\section{ECONOMIC BACKGROUND}

Families' finances are affected by both their own decisions and the state of the broader economy. Over the 2004-07 period, real gross domestic product (GDP) increased, on average, about 2.5 percent per

2. See box "The Data Used in This Article" for a general description of the data. The appendix to this article provides a summary of key technical aspects of the survey. See also Bucks, Kennickell, and Moore, "Recent Changes in U.S. Family Finances." year. However, toward the end of 2007, the pace of economic activity slowed noticeably. The unemployment rate stood at 5.5 percent in mid-2004, fell to 4.5 percent by late 2006 , and then increased to 5.0 percent at the end of 2007. The rate of inflation, as measured by the consumer price index for all urban consumers (CPI-U-RS), increased somewhat over the period, from an annual average of 2.7 percent in 2004 to 2.9 percent in 2007 ; the increase was driven, in part, by the escalation of food and energy prices.

Developments in financial markets over the threeyear period were varied. The major stock market indexes climbed over most of the period before beginning a decline in late 2007; from September 2004 to September 2007, the Wilshire 5000 index rose 41.7 percent. Interest rates on new consumer loans generally increased; for example, the interest rate on a new 30-year fixed-rate mortgage averaged 5.75 percent in September 2004, when about one-half of the interviews for the 2004 survey had been completed, and was 6.38 percent three years later. Yields also rose on liquid deposits, time deposits, and bonds; for example, the rate on a three-month certificate of deposit rose from an average of 1.86 percent in September 2004 to 5.46 percent in September 2007.

The national purchase-only LoanPerformance Home Price Index, produced by First American CoreLogic, increased more than 12.4 percent between September 2004 and September 2007. Price increases varied sharply across areas of the country. The largest increase in the index was a 49.9 percent rise for Hawaii. While most states saw an increase, the index declined 8.0 percent for Michigan and by smaller amounts for Ohio, Rhode Island, and Massachusetts. Homeownership rates were little changed over the period after a long and steady increase. Nonetheless, the number of homeowners rose with population growth, and subprime mortgages are generally thought to have played an important part in financing home purchases.

No major tax legislation was passed during the period, but other important institutional changes occurred. The Bankruptcy Abuse Prevention and Consumer Protection Act of April 2005 altered the rules for liquidation of consumers' liabilities under bankruptcy. In particular, the new rules require that consumers with a certain level of income pay back at least part of their outstanding debts, whereas in the past the entire amount might have been liquidated. The law also mandated financial counseling for anyone declaring bankruptcy. Continuing innovation in financial markets over the period supported further 
proliferation of hedge funds and other sophisticated instruments for money management.

Several demographic shifts had important consequences for the structure of the population. The aging of the baby-boom population from 2004 to 2007 drove a 12.5 percent increase in the population aged 55 to 64 . Overall population growth was about 2.9 percent, and, according to figures from the U.S. Census Bureau, 37.3 percent of that growth was due to net immigration. Also according to Census Bureau estimates, the number of households increased 2.3 percent - about the same pace as in the 2001-04 periodand the average number of persons per household rose slightly, from 2.59 people in 2004 to 2.61 in 2007.

Only a small fraction of the 2007 SCF interviews took place in 2008. Thus, the survey data are largely unaffected by the declines in economic activity in 2008 , the fall in the market price of corporate equities, and the continued slide in house prices. Nonetheless, readers' views of the survey results may be colored by the knowledge that, in the first three quarters of 2008, a broad measure of the value of corporate equities declined more than one-third, and house prices overall declined approximately an additional 5 percent. At a few places in the article, an attempt is made to gauge the first-order effects of these changes on families' finances.

\section{INCOME}

The change in real before-tax family income between 2004 and 2007 diverged from the pattern seen in the preceding three-year period. ${ }^{3}$ While median income declined slightly over the more recent period, the mean rose 8.5 percent (table 1). ${ }^{4}$ Over the preceding

\footnotetext{
3. To measure income, the interviewers request information on the family's cash income, before taxes, for the full calendar year preceding the survey. The components of income in the SCF are wages; self-employment and business income; taxable and tax-exempt interest; dividends; realized capital gains; food stamps and other, related support programs provided by government; pensions and withdrawals from retirement accounts; Social Security; alimony and other support payments; and miscellaneous sources of income for all members of the primary economic unit in the household.

4. Over the 2004-07 period, estimates of inflation-adjusted household income for the previous year from the Current Population Survey (CPS) of the Census Bureau show an increase in both the median (1.4 percent) and the mean (2.7 percent). Typically, the SCF shows a higher level of mean income than does the CPS; for 2007, the SCF yields an estimate of $\$ 84,300$, while the CPS yields an estimate of $\$ 68,400$. As discussed in more detail in the appendix, the two surveys differ in their definitions of the units of observation and in other aspects of their methodologies. Most relevant here is the fact that a CPS household can contain more people than a corresponding SCF family. If the SCF measure is expanded to include income of household members not included in the SCF definition of a family, the median rises 2.7 percent (to $\$ 49,400$ ) over the three-year period, and
}

three-year period, the median had increased 1.7 percent, and the mean had declined 2.3 percent. The changes for both periods stand in much stronger contrast to a pattern of substantial increases in both the median and the mean dating to the early 1990s.

Underlying the recent change was a shift in the composition of income between 2004 and 2007 (table 2). The share of family income attributable to wages and salaries fell 5.2 percentage points over the period, which approximately balanced a 3.5 percentage point rise in the share of realized capital gains and a 2.7 percentage point increase in income from self-employment, a farm, or a business. These shifts were seen across all wealth groups except the group between the 75th and 90th percentiles. As may be seen across the years shown in the table, wage income tends to be a smaller factor for the highest wealth group.

Some patterns of income distribution hold generally across the years of SCF data shown in table 1.5 Across age classes, median and mean incomes show a life-cycle pattern, rising to a peak in the middle age groups and then declining for groups that are older and increasingly more likely to be retired. Couples tend to have higher incomes than single persons, in part because couples have more potential wage earners. Income also shows a strong positive association with education; in particular, incomes for families headed by a person who has a college degree are substantially higher than for those with any lesser amount of schooling. Incomes of white non-Hispanic families are substantially higher than those of other families. ${ }^{6}$ Families headed by a self-employed worker consistently have the highest median and mean incomes of all work-status groups. Families headed by a person in a managerial or professional

the mean rises 11.0 percent (to $\$ 86,900$ ). The substantial difference in mean levels is likely the result of the truncation of large values in the CPS data above a certain amount, which is done with the intent of minimizing the possibility that participants in that survey might be identifiable.

5. Tabular information from the survey beyond that presented in this article is available at www.federalreserve.gov/pubs/oss/oss $2 / 2007 /$ scf2007home.html. This information includes versions of all of the numbered tables in this article, for all of the surveys from 1989 to 2007 where the underlying information is available. Mean values for the demographic groups reported in this article are also provided. The estimates of the means, however, are more likely to be affected by sampling error than are the estimates of the medians. In addition, some alternative versions of the tables in this article are given. For those who wish to make further alternative calculations, this website provides a utility ("tabling wizard") that may be used to compute estimates of customized tables based on the variables analyzed in this article, as well as data files that may be used as inputs to more sophisticated statistical software.

6. See the appendix for a discussion of racial and ethnic identification in the SCF. 
1. Before-tax family income, percentage of families that saved, and distribution of families, by selected characteristics of families, 1998-2007 surveys

Thousands of 2007 dollars except as noted

\begin{tabular}{|c|c|c|c|c|c|c|c|c|}
\hline \multirow{3}{*}{ Family characteristic } & \multicolumn{4}{|c|}{1998} & \multicolumn{4}{|c|}{2001} \\
\hline & \multicolumn{2}{|c|}{ Income } & \multirow{2}{*}{$\begin{array}{l}\text { Percentage } \\
\text { of families } \\
\text { that saved }\end{array}$} & \multirow{2}{*}{$\begin{array}{l}\text { Percentage } \\
\text { of families }\end{array}$} & \multicolumn{2}{|c|}{ Income } & \multirow{2}{*}{$\begin{array}{l}\text { Percentage } \\
\text { of families } \\
\text { that saved }\end{array}$} & \multirow{2}{*}{$\begin{array}{l}\text { Percentage } \\
\text { of families }\end{array}$} \\
\hline & Median & Mean & & & Median & Mean & & \\
\hline All families $\ldots \ldots \ldots \ldots \ldots \ldots \ldots \ldots$ & $\begin{array}{c}\mathbf{4 2 . 6} \\
(1.0)\end{array}$ & $\begin{array}{c}\mathbf{6 7 . 7} \\
(1.4)\end{array}$ & 55.9 & 100.0 & $\begin{array}{r}46.7 \\
(.9)\end{array}$ & $\begin{array}{c}\mathbf{7 9 . 5} \\
(2.3)\end{array}$ & 59.2 & 100.0 \\
\hline 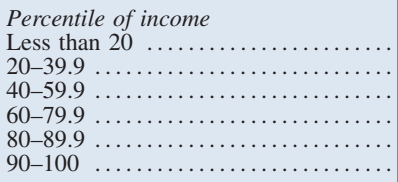 & $\begin{array}{r}10.5 \\
25.8 \\
42.6 \\
67.8 \\
100.6 \\
166.3\end{array}$ & $\begin{array}{r}10.1 \\
25.7 \\
43.3 \\
69.1 \\
101.3 \\
279.5\end{array}$ & $\begin{array}{l}32.1 \\
45.5 \\
56.1 \\
67.9 \\
73.7 \\
82.0\end{array}$ & $\begin{array}{l}20.0 \\
20.0 \\
20.0 \\
20.0 \\
10.0 \\
10.0\end{array}$ & $\begin{array}{r}12.0 \\
28.5 \\
46.7 \\
75.8 \\
115.4 \\
198.3\end{array}$ & $\begin{array}{r}11.7 \\
28.2 \\
47.1 \\
76.2 \\
114.7 \\
354.1\end{array}$ & $\begin{array}{l}30.0 \\
53.4 \\
61.3 \\
72.0 \\
74.9 \\
84.3\end{array}$ & $\begin{array}{l}20.0 \\
20.0 \\
20.0 \\
20.0 \\
10.0 \\
10.0\end{array}$ \\
\hline 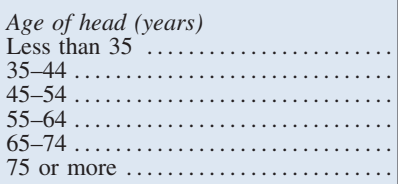 & $\begin{array}{l}34.9 \\
53.6 \\
64.5 \\
49.1 \\
31.0 \\
21.3\end{array}$ & $\begin{array}{l}46.0 \\
76.4 \\
88.9 \\
91.4 \\
59.5 \\
37.2\end{array}$ & $\begin{array}{l}53.0 \\
57.3 \\
57.8 \\
61.1 \\
56.3 \\
48.6\end{array}$ & $\begin{array}{l}23.3 \\
23.3 \\
19.2 \\
12.8 \\
11.2 \\
10.2\end{array}$ & $\begin{array}{l}39.1 \\
60.1 \\
63.7 \\
52.9 \\
32.5 \\
26.2\end{array}$ & $\begin{array}{r}51.7 \\
90.2 \\
109.0 \\
101.7 \\
68.0 \\
43.0\end{array}$ & $\begin{array}{l}52.9 \\
62.3 \\
61.7 \\
62.0 \\
61.8 \\
55.5\end{array}$ & $\begin{array}{l}22.7 \\
22.3 \\
20.6 \\
13.2 \\
10.7 \\
10.4\end{array}$ \\
\hline $\begin{array}{l}\text { Family structure } \\
\text { Single with child(ren) } \ldots \ldots \ldots \ldots \ldots \\
\text { Single, no child, age less than } 55 \ldots \ldots \\
\text { Single, no child, age } 55 \text { or more } \ldots \ldots \\
\text { Couple with child(ren) } \ldots \ldots \ldots \ldots \ldots \ldots \\
\text { Couple, no child } \ldots \ldots \ldots \ldots \ldots \ldots \ldots \ldots\end{array}$ & $\begin{array}{l}25.8 \\
29.7 \\
21.0 \\
64.5 \\
61.4\end{array}$ & $\begin{array}{l}33.6 \\
37.6 \\
33.0 \\
85.6 \\
92.0\end{array}$ & $\begin{array}{l}42.1 \\
48.3 \\
47.8 \\
62.1 \\
62.1\end{array}$ & $\begin{array}{r}6.8 \\
20.4 \\
14.3 \\
12.3 \\
46.2\end{array}$ & $\begin{array}{l}28.4 \\
31.5 \\
19.7 \\
66.1 \\
67.1\end{array}$ & $\begin{array}{r}36.1 \\
43.5 \\
37.9 \\
98.6 \\
106.8\end{array}$ & $\begin{array}{l}47.3 \\
52.5 \\
49.4 \\
63.3 \\
65.3\end{array}$ & $\begin{array}{r}6.0 \\
20.4 \\
13.3 \\
11.8 \\
48.5\end{array}$ \\
\hline $\begin{array}{l}\text { Education of head } \\
\text { No high school diploma } \ldots \ldots \ldots \ldots \ldots \\
\text { High school diploma } \ldots \ldots \ldots \ldots \ldots \ldots \\
\text { Some college } \ldots \ldots \ldots \ldots \ldots \ldots \ldots \ldots \ldots \\
\text { College degree } \ldots \ldots \ldots \ldots \ldots \ldots \ldots \ldots\end{array}$ & $\begin{array}{l}19.8 \\
37.2 \\
45.2 \\
70.0\end{array}$ & $\begin{array}{r}27.6 \\
47.1 \\
64.7 \\
109.0\end{array}$ & $\begin{array}{l}39.5 \\
53.7 \\
56.7 \\
65.6\end{array}$ & $\begin{array}{l}16.5 \\
31.9 \\
18.5 \\
33.2\end{array}$ & $\begin{array}{l}19.8 \\
39.7 \\
47.9 \\
79.4\end{array}$ & $\begin{array}{r}29.4 \\
52.4 \\
64.9 \\
136.4\end{array}$ & $\begin{array}{l}38.7 \\
56.7 \\
61.7 \\
70.0\end{array}$ & $\begin{array}{l}16.0 \\
31.7 \\
18.3 \\
34.0\end{array}$ \\
\hline $\begin{array}{l}\text { Race or ethnicity of respondent } \\
\text { White non-Hispanic } \ldots \ldots \ldots \ldots \ldots \ldots \\
\text { Nonwhite or Hispanic } \ldots \ldots \ldots \ldots \ldots \ldots\end{array}$ & $\begin{array}{l}48.6 \\
29.7\end{array}$ & $\begin{array}{l}75.4 \\
42.3\end{array}$ & $\begin{array}{l}60.0 \\
42.3\end{array}$ & $\begin{array}{l}76.8 \\
23.2\end{array}$ & $\begin{array}{l}52.9 \\
30.1\end{array}$ & $\begin{array}{l}90.0 \\
47.6\end{array}$ & $\begin{array}{l}63.1 \\
47.4\end{array}$ & $\begin{array}{l}75.4 \\
24.6\end{array}$ \\
\hline $\begin{array}{l}\text { Current work status of head } \\
\text { Working for someone else } \ldots \ldots \ldots \ldots \\
\text { Self-employed } \ldots \ldots \ldots \ldots \ldots \ldots \ldots \ldots \\
\text { Retired } \ldots \ldots \ldots \ldots \ldots \ldots \ldots \ldots \ldots \\
\text { Other not working } \ldots \ldots \ldots \ldots \ldots \ldots \ldots\end{array}$ & $\begin{array}{l}51.6 \\
67.1 \\
24.5 \\
14.8\end{array}$ & $\begin{array}{r}68.2 \\
139.2 \\
42.0 \\
27.7\end{array}$ & $\begin{array}{l}59.8 \\
61.1 \\
48.7 \\
33.3\end{array}$ & $\begin{array}{r}59.2 \\
11.3 \\
24.4 \\
5.1\end{array}$ & $\begin{array}{l}55.3 \\
74.1 \\
24.6 \\
19.3\end{array}$ & $\begin{array}{r}78.8 \\
161.8 \\
46.8 \\
42.6\end{array}$ & $\begin{array}{l}61.6 \\
70.4 \\
50.6 \\
42.3\end{array}$ & $\begin{array}{r}60.9 \\
11.7 \\
22.9 \\
4.5\end{array}$ \\
\hline $\begin{array}{l}\text { Current occupation of head } \\
\text { Managerial or professional } \ldots \ldots \ldots \ldots \\
\text { Technical, sales, or services } \ldots \ldots \ldots \ldots \\
\text { Other occupation } \ldots \ldots \ldots \ldots \ldots \ldots \ldots \\
\text { Retired or other not working } \ldots \ldots \ldots \ldots\end{array}$ & $\begin{array}{l}77.5 \\
39.1 \\
47.8 \\
22.6\end{array}$ & $\begin{array}{r}123.1 \\
59.7 \\
54.2 \\
39.7\end{array}$ & $\begin{array}{l}68.4 \\
55.6 \\
55.6 \\
46.1\end{array}$ & $\begin{array}{l}24.2 \\
21.0 \\
25.3 \\
29.5\end{array}$ & $\begin{array}{l}83.2 \\
42.1 \\
48.1 \\
24.2\end{array}$ & $\begin{array}{r}146.4 \\
62.3 \\
57.3 \\
46.1\end{array}$ & $\begin{array}{l}72.4 \\
58.2 \\
56.6 \\
49.2\end{array}$ & $\begin{array}{l}27.1 \\
23.7 \\
21.8 \\
27.4\end{array}$ \\
\hline 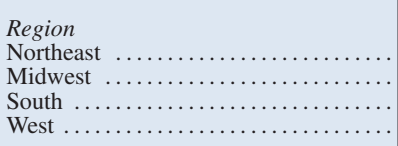 & $\begin{array}{l}45.2 \\
41.9 \\
40.2 \\
46.1\end{array}$ & $\begin{array}{l}77.6 \\
62.4 \\
63.0 \\
72.7\end{array}$ & $\begin{array}{l}53.5 \\
58.3 \\
55.0 \\
56.9\end{array}$ & $\begin{array}{l}19.3 \\
23.6 \\
35.7 \\
21.3\end{array}$ & $\begin{array}{l}48.3 \\
51.3 \\
42.1 \\
47.6\end{array}$ & $\begin{array}{l}90.9 \\
75.7 \\
71.8 \\
86.6\end{array}$ & $\begin{array}{l}58.1 \\
63.0 \\
57.3 \\
59.5\end{array}$ & $\begin{array}{l}19.0 \\
23.0 \\
36.2 \\
21.8\end{array}$ \\
\hline $\begin{array}{l}\text { Urbanicity } \\
\text { Metropolitan statistical area (MSA) } \ldots \\
\text { Non-MSA } \ldots \ldots \ldots \ldots \ldots \ldots \ldots \ldots \ldots\end{array}$ & $\begin{array}{l}45.2 \\
35.6\end{array}$ & $\begin{array}{l}71.9 \\
43.2\end{array}$ & $\begin{array}{l}56.3 \\
53.6\end{array}$ & $\begin{array}{l}85.3 \\
14.7\end{array}$ & $\begin{array}{l}48.1 \\
35.4\end{array}$ & $\begin{array}{l}84.6 \\
47.9\end{array}$ & $\begin{array}{l}59.7 \\
56.3\end{array}$ & $\begin{array}{l}86.2 \\
13.8\end{array}$ \\
\hline $\begin{array}{l}\text { Housing status } \\
\text { Owner } \ldots \ldots \ldots \ldots \ldots \ldots \ldots \ldots \ldots \ldots \\
\text { Renter or other } \ldots \ldots \ldots \ldots \ldots \ldots \ldots \ldots\end{array}$ & $\begin{array}{l}55.7 \\
25.8\end{array}$ & $\begin{array}{l}84.9 \\
34.0\end{array}$ & $\begin{array}{l}62.2 \\
43.4\end{array}$ & $\begin{array}{l}66.2 \\
33.8\end{array}$ & $\begin{array}{l}60.9 \\
28.9\end{array}$ & $\begin{array}{l}99.5 \\
37.7\end{array}$ & $\begin{array}{l}66.7 \\
43.6\end{array}$ & $\begin{array}{l}67.7 \\
32.3\end{array}$ \\
\hline 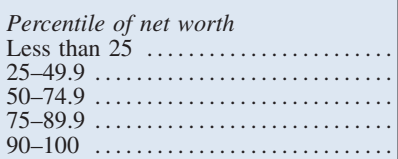 & $\begin{array}{r}20.3 \\
38.7 \\
51.6 \\
72.3 \\
112.5\end{array}$ & $\begin{array}{r}25.9 \\
43.1 \\
59.6 \\
86.0 \\
226.6\end{array}$ & $\begin{array}{l}36.3 \\
50.3 \\
61.8 \\
72.0 \\
80.0\end{array}$ & $\begin{array}{l}25.0 \\
25.0 \\
25.0 \\
15.0 \\
10.0\end{array}$ & $\begin{array}{r}23.0 \\
40.9 \\
59.8 \\
81.4 \\
147.9\end{array}$ & $\begin{array}{r}28.1 \\
46.2 \\
68.9 \\
91.9 \\
299.5\end{array}$ & $\begin{array}{l}34.5 \\
54.2 \\
68.2 \\
77.4 \\
84.1\end{array}$ & $\begin{array}{l}25.0 \\
25.0 \\
25.0 \\
15.0 \\
10.0\end{array}$ \\
\hline
\end{tabular}

NotE: For questions on income, respondents were asked to base their answers on the calendar year preceding the interview. For questions on saving, respondents were asked to base their answers on the 12 months preceding the interview.

Percentage distributions may not sum to 100 because of rounding. Dollars have been converted to 2007 values with the current-methods consumer price index for all urban consumers (see box "The Data Used in This Article"). See the appendix for details on standard errors (shown in parentheses below the first row of data for the means and medians here and in table 4) and for definitions of family and family head. 
1. Before-tax family income, percentage of families that saved, and distribution of families, by selected characteristics of families, 1998-2007 surveys-Continued

Thousands of 2007 dollars except as noted

\begin{tabular}{|c|c|c|c|c|c|c|c|c|}
\hline \multirow{3}{*}{ Family characteristic } & \multicolumn{4}{|c|}{2004} & \multicolumn{4}{|c|}{2007} \\
\hline & \multicolumn{2}{|c|}{ Income } & \multirow{2}{*}{$\begin{array}{l}\text { Percentage } \\
\text { of families } \\
\text { that saved }\end{array}$} & \multirow{2}{*}{$\begin{array}{l}\text { Percentage } \\
\text { of families }\end{array}$} & \multicolumn{2}{|c|}{ Income } & \multirow{2}{*}{$\begin{array}{l}\text { Percentage } \\
\text { of families } \\
\text { that saved }\end{array}$} & \multirow{2}{*}{$\begin{array}{l}\text { Percentage } \\
\text { of families }\end{array}$} \\
\hline & Median & Mean & & & Median & Mean & & \\
\hline All families $\ldots \ldots \ldots \ldots \ldots \ldots$ & $\begin{array}{r}47.5 \\
(.9)\end{array}$ & $\begin{array}{c}77.7 \\
(1.3)\end{array}$ & 56.1 & 100.0 & $\begin{array}{r}\mathbf{4 7 . 3} \\
(.8)\end{array}$ & $\begin{array}{c}\mathbf{8 4 . 3} \\
(1.3)\end{array}$ & 56.5 & 100.0 \\
\hline 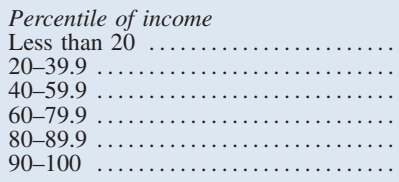 & $\begin{array}{r}12.2 \\
28.2 \\
47.5 \\
74.9 \\
115.1 \\
203.0\end{array}$ & $\begin{array}{r}11.9 \\
28.6 \\
47.7 \\
76.0 \\
117.0 \\
331.9\end{array}$ & $\begin{array}{l}34.0 \\
43.3 \\
54.5 \\
69.3 \\
77.8 \\
80.6\end{array}$ & $\begin{array}{l}20.0 \\
20.0 \\
20.0 \\
20.0 \\
10.0 \\
10.0\end{array}$ & $\begin{array}{r}12.3 \\
28.8 \\
47.3 \\
75.1 \\
114.0 \\
206.9\end{array}$ & $\begin{array}{r}12.3 \\
28.3 \\
47.3 \\
76.6 \\
116.0 \\
397.7\end{array}$ & $\begin{array}{l}33.7 \\
45.1 \\
57.8 \\
66.8 \\
72.9 \\
84.8\end{array}$ & $\begin{array}{l}20.0 \\
20.0 \\
20.0 \\
20.0 \\
10.0 \\
10.0\end{array}$ \\
\hline 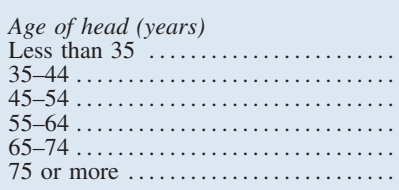 & $\begin{array}{l}36.1 \\
54.9 \\
67.1 \\
59.8 \\
36.6 \\
26.0\end{array}$ & $\begin{array}{r}49.6 \\
81.1 \\
103.6 \\
110.2 \\
65.6 \\
44.9\end{array}$ & $\begin{array}{l}55.0 \\
58.0 \\
58.5 \\
58.5 \\
57.1 \\
45.7\end{array}$ & $\begin{array}{l}22.2 \\
20.6 \\
20.8 \\
15.2 \\
10.5 \\
10.7\end{array}$ & $\begin{array}{l}37.4 \\
56.6 \\
64.2 \\
54.6 \\
39.0 \\
22.8\end{array}$ & $\begin{array}{r}51.7 \\
83.7 \\
112.4 \\
111.2 \\
92.4 \\
45.7\end{array}$ & $\begin{array}{l}58.9 \\
56.4 \\
55.8 \\
58.4 \\
56.7 \\
49.4\end{array}$ & $\begin{array}{l}21.7 \\
19.6 \\
20.8 \\
16.8 \\
10.5 \\
10.6\end{array}$ \\
\hline $\begin{array}{l}\text { Family structure } \\
\text { Single with child(ren) } \ldots \ldots \ldots \ldots \ldots \\
\text { Single, no child, age less than } 55 \ldots \ldots \\
\text { Single, no child, age } 55 \text { or more } \ldots \ldots \\
\text { Couple with child(ren) } \ldots \ldots \ldots \ldots \ldots \ldots \\
\text { Couple, no child } \ldots \ldots \ldots \ldots \ldots \ldots \ldots \ldots\end{array}$ & $\begin{array}{l}31.6 \\
30.5 \\
23.4 \\
71.1 \\
67.7\end{array}$ & $\begin{array}{r}38.1 \\
40.8 \\
37.4 \\
99.8 \\
107.4\end{array}$ & $\begin{array}{l}40.7 \\
49.2 \\
46.0 \\
61.6 \\
63.3\end{array}$ & $\begin{array}{r}7.2 \\
20.0 \\
14.8 \\
12.6 \\
45.4\end{array}$ & $\begin{array}{l}30.9 \\
30.9 \\
24.6 \\
67.9 \\
66.5\end{array}$ & $\begin{array}{r}46.0 \\
44.9 \\
36.3 \\
105.4 \\
116.2\end{array}$ & $\begin{array}{l}45.8 \\
50.1 \\
48.0 \\
61.8 \\
62.0\end{array}$ & $\begin{array}{r}6.4 \\
19.3 \\
15.4 \\
12.3 \\
46.5\end{array}$ \\
\hline $\begin{array}{l}\text { Education of head } \\
\text { No high school diploma } \ldots \ldots \ldots \ldots \ldots \\
\text { High school diploma } \ldots \ldots \ldots \ldots \ldots \ldots \\
\text { Some college } \ldots \ldots \ldots \ldots \ldots \ldots \ldots \ldots \ldots \\
\text { College degree } \ldots \ldots \ldots \ldots \ldots \ldots \ldots\end{array}$ & $\begin{array}{l}21.3 \\
39.3 \\
45.1 \\
80.5\end{array}$ & $\begin{array}{r}28.5 \\
49.2 \\
61.6 \\
129.1\end{array}$ & $\begin{array}{l}35.9 \\
54.0 \\
51.0 \\
68.3\end{array}$ & $\begin{array}{l}14.4 \\
30.6 \\
18.4 \\
36.6\end{array}$ & $\begin{array}{l}22.2 \\
36.7 \\
45.6 \\
78.2\end{array}$ & $\begin{array}{r}31.3 \\
51.1 \\
68.1 \\
143.8\end{array}$ & $\begin{array}{l}41.6 \\
51.1 \\
53.6 \\
68.6\end{array}$ & $\begin{array}{l}13.5 \\
32.9 \\
18.4 \\
35.3\end{array}$ \\
\hline $\begin{array}{l}\text { Race or ethnicity of respondent } \\
\text { White non-Hispanic } \ldots \ldots \ldots \ldots \ldots \ldots \\
\text { Nonwhite or Hispanic } \ldots \ldots \ldots \ldots \ldots \ldots\end{array}$ & $\begin{array}{l}54.3 \\
32.7\end{array}$ & $\begin{array}{l}88.6 \\
49.4\end{array}$ & $\begin{array}{l}60.1 \\
45.6\end{array}$ & $\begin{array}{l}72.2 \\
27.8\end{array}$ & $\begin{array}{l}51.8 \\
36.8\end{array}$ & $\begin{array}{l}96.9 \\
53.7\end{array}$ & $\begin{array}{l}58.8 \\
50.8\end{array}$ & $\begin{array}{l}70.7 \\
29.3\end{array}$ \\
\hline $\begin{array}{l}\text { Current work status of head } \\
\text { Working for someone else } \ldots \ldots \ldots \ldots \\
\text { Self-employed } \ldots \ldots \ldots \ldots \ldots \ldots \ldots \ldots \\
\text { Retired } \ldots \ldots \ldots \ldots \ldots \ldots \ldots \ldots \ldots \ldots \ldots \\
\text { Other not working } \ldots \ldots \ldots \ldots \ldots \ldots \ldots\end{array}$ & $\begin{array}{l}54.1 \\
73.3 \\
26.8 \\
22.6\end{array}$ & $\begin{array}{r}77.0 \\
155.5 \\
47.5 \\
41.0\end{array}$ & $\begin{array}{l}59.2 \\
68.7 \\
44.0 \\
44.9\end{array}$ & $\begin{array}{r}60.1 \\
11.8 \\
23.7 \\
4.4\end{array}$ & $\begin{array}{l}56.6 \\
75.7 \\
24.7 \\
20.4\end{array}$ & $\begin{array}{r}83.1 \\
191.8 \\
51.1 \\
35.4\end{array}$ & $\begin{array}{l}60.3 \\
62.8 \\
46.6 \\
45.4\end{array}$ & $\begin{array}{r}59.9 \\
10.5 \\
25.0 \\
4.6\end{array}$ \\
\hline $\begin{array}{l}\text { Current occupation of head } \\
\text { Managerial or professional } \ldots \ldots \ldots \ldots \\
\text { Technical, sales, or services } \ldots \ldots \ldots \ldots \\
\text { Other occupation } \ldots \ldots \ldots \ldots \ldots \ldots \\
\text { Retired or other not working } \ldots \ldots \ldots \ldots\end{array}$ & $\begin{array}{l}84.8 \\
41.1 \\
49.6 \\
26.2\end{array}$ & $\begin{array}{r}140.9 \\
58.3 \\
55.6 \\
46.5\end{array}$ & $\begin{array}{l}67.7 \\
55.4 \\
57.3 \\
44.1\end{array}$ & $\begin{array}{l}28.3 \\
22.1 \\
21.6 \\
28.1\end{array}$ & $\begin{array}{l}85.4 \\
44.2 \\
49.4 \\
23.8\end{array}$ & $\begin{array}{r}156.1 \\
67.6 \\
57.9 \\
48.7\end{array}$ & $\begin{array}{l}70.2 \\
55.6 \\
53.6 \\
46.4\end{array}$ & $\begin{array}{l}27.5 \\
21.8 \\
21.1 \\
29.6\end{array}$ \\
\hline 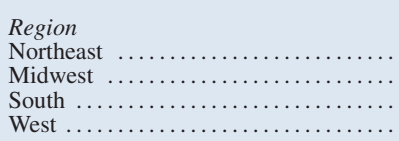 & $\begin{array}{l}55.9 \\
49.6 \\
40.6 \\
50.7\end{array}$ & $\begin{array}{l}96.1 \\
74.1 \\
68.0 \\
81.9\end{array}$ & $\begin{array}{l}59.5 \\
59.9 \\
52.5 \\
55.2\end{array}$ & $\begin{array}{l}18.8 \\
22.9 \\
36.3 \\
22.0\end{array}$ & $\begin{array}{l}51.4 \\
44.2 \\
42.9 \\
51.9\end{array}$ & $\begin{array}{r}100.4 \\
74.9 \\
79.3 \\
88.7\end{array}$ & $\begin{array}{l}53.5 \\
58.2 \\
56.9 \\
56.3\end{array}$ & $\begin{array}{l}18.3 \\
22.8 \\
36.7 \\
22.1\end{array}$ \\
\hline $\begin{array}{l}\text { Urbanicity } \\
\text { Metropolitan statistical area (MSA) } \ldots \\
\text { Non-MSA } \ldots \ldots \ldots \ldots \ldots \ldots \ldots \ldots \ldots\end{array}$ & $\begin{array}{l}50.8 \\
32.8\end{array}$ & $\begin{array}{l}84.5 \\
45.0\end{array}$ & $\begin{array}{l}56.9 \\
52.3\end{array}$ & $\begin{array}{l}82.9 \\
17.1\end{array}$ & $\begin{array}{l}50.4 \\
36.0\end{array}$ & $\begin{array}{l}91.3 \\
50.2\end{array}$ & $\begin{array}{l}57.0 \\
54.0\end{array}$ & $\begin{array}{l}82.9 \\
17.1\end{array}$ \\
\hline $\begin{array}{l}\text { Housing status } \\
\text { Owner } \ldots \ldots \ldots \ldots \ldots \ldots \ldots \ldots \ldots \ldots \\
\text { Renter or other } \ldots \ldots \ldots \ldots \ldots \ldots \ldots \ldots\end{array}$ & $\begin{array}{l}60.6 \\
27.1\end{array}$ & $\begin{array}{l}96.0 \\
37.0\end{array}$ & $\begin{array}{l}62.3 \\
42.3\end{array}$ & $\begin{array}{l}69.1 \\
30.9\end{array}$ & $\begin{array}{l}61.7 \\
27.8\end{array}$ & $\begin{array}{r}105.6 \\
37.5\end{array}$ & $\begin{array}{l}60.9 \\
46.7\end{array}$ & $\begin{array}{l}68.6 \\
31.4\end{array}$ \\
\hline 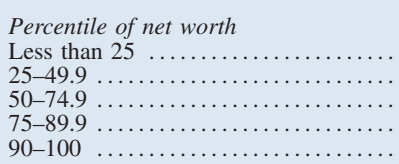 & $\begin{array}{r}22.6 \\
40.6 \\
57.5 \\
84.6 \\
157.9\end{array}$ & $\begin{array}{r}27.5 \\
46.4 \\
66.5 \\
96.5 \\
281.4\end{array}$ & $\begin{array}{l}34.8 \\
53.6 \\
62.2 \\
72.4 \\
76.0\end{array}$ & $\begin{array}{l}25.0 \\
25.0 \\
25.0 \\
15.0 \\
10.0\end{array}$ & $\begin{array}{r}23.6 \\
41.0 \\
56.7 \\
82.3 \\
158.4\end{array}$ & $\begin{array}{r}29.2 \\
46.5 \\
66.6 \\
92.9 \\
347.5\end{array}$ & $\begin{array}{l}40.4 \\
52.9 \\
59.0 \\
69.0 \\
80.2\end{array}$ & $\begin{array}{l}25.0 \\
25.0 \\
25.0 \\
15.0 \\
10.0\end{array}$ \\
\hline
\end{tabular}




\section{The Data Used in This Article}

Data from the Survey of Consumer Finances (SCF) are the basis of the analysis presented in this article. The SCF is a triennial interview survey of U.S. families sponsored by the Board of Governors of the Federal Reserve System with the cooperation of the U.S. Department of the Treasury. Since 1992, data for the SCF have been collected by NORC, a research organization at the University of Chicago, roughly between May and December of each survey year.

The majority of statistics included in this article are related to characteristics of "families." As used here, this term is more comparable with the U.S. Census Bureau definition of "households" than with its use of "families," which excludes the possibility of one-person families. The appendix provides full definitions of "family" for the SCF and the associated family "head." The survey collects information on families' total income before taxes for the calendar year preceding the survey. But the bulk of the data cover the status of families as of the time of the interview, including detailed information on their balance sheets and use of financial services as well as on their pensions, labor force participation, and demographic characteristics. Except in a small number of instances (see the appendix and the text for details), the survey questionnaire has changed in only minor ways relevant to this article since 1989, and every effort has been made to ensure the maximum degree of comparability of the data over time.

The need to measure financial characteristics imposes special requirements on the sample design for the survey. The SCF is expected to provide reliable information both on attributes that are broadly distributed in the population (such as homeownership) and on those that are highly concentrated in a relatively small part of the population (such as closely held businesses). To address this requirement, the SCF employs a sample design, essentially unchanged since 1989, consisting of two parts: a standard, geographically based random sample and a special oversample of relatively wealthy families. Weights are used to combine information from the two samples to make estimates for the full population. In the 2007 survey, 4,422 families were interviewed, and in the 2004 survey, 4,522 were interviewed.

This article draws principally upon the final data from the 2007 and 2004 surveys. To provide a larger context, some information is also included from the final versions of earlier surveys. ${ }^{1}$ Differences between estimates from earlier surveys as reported here and as reported in earlier Federal Reserve Bulletin articles are attributable to additional statistical processing, correction of minor data errors, revisions to the survey weights, conceptual changes in the definitions of variables used in the articles, and adjustments for inflation. In this article, all dollar amounts from the SCF are adjusted to 2007 dollars using the "current methods" version of the consumer price index for all urban consumers (CPI-U-RS). The appendix provides additional detail on the adjustments.

The principal detailed tables describing asset and debt holdings focus on the percentage of various groups that have such items and the median holding for those that have them. ${ }^{2}$ This conditional median is chosen to give a sense of the "typical" holding. Generally, when one deals with data that exhibit very large values for a relatively small part of the population-as is the case for many of the items considered in this article-estimates of the median are often statistically less sensitive to such outliers than are estimates of the mean.

One liability of using the median as a descriptive device is that medians are not additive; that is, the sum of the medians of two items for the same population is not generally equal to the median of the sum (for example, median assets less median liabilities does not equal median net worth). In contrast, means for a common population are additive. Where a comparable median and mean are given, the gain of the mean relative to the median may usually be taken as indicative of relatively greater change at the top of the distribution; for example, when the mean increases more rapidly than the median, it is typically taken to indicate that the values in the top of the distribution rose more rapidly than those in the lower part of the distribution.

To provide a measure of the significance of the developments discussed in this article, standard errors due to sampling and imputation for missing data are given for selected estimates. Space limits prevent the inclusion of the standard errors for all estimates. Although we do not directly address the statistical significance of the results, the article highlights findings that are significant or are interesting in a broader context.

1. Additional information about the survey is available at www.federalreserve.gov/pubs/oss/oss2/2007/scf2007home.html.

2. The median of a distribution is defined as the value at which equal parts of the population considered have values larger or smaller. 
2. Amount of before-tax family income, distributed by income sources, by percentile of net worth, 2004 and 2007 surveys Percent

\begin{tabular}{|c|c|c|c|c|c|c|}
\hline \multirow{2}{*}{ Income source } & \multicolumn{5}{|c|}{ Percentile of net worth } & \multirow{2}{*}{ All families } \\
\hline & Less than 25 & $25-49.9$ & $50-74.9$ & $75-89.9$ & $90-100$ & \\
\hline 2004 Survey of Consumer Finances & & & & & & \\
\hline Wages $\ldots \ldots \ldots \ldots \ldots \ldots \ldots \ldots$ & 82.1 & 85.4 & 79.3 & 72.4 & 53.0 & 69.7 \\
\hline Interest or dividends . . . . . . . & $\dagger$ & .3 & .7 & 1.8 & 8.2 & 3.5 \\
\hline Business, farm, self-employment & 1.1 & 2.7 & 5.0 & 8.5 & 21.5 & 10.9 \\
\hline Capital gains .................. & $\dagger$ & $\dagger$ & $\dagger$ & 1.2 & 8.3 & 3.2 \\
\hline Social Security or retirement & 9.6 & 9.2 & 13.2 & 15.4 & 8.2 & 10.9 \\
\hline Transfers or other ........... & 7.2 & 2.5 & 1.7 & .7 & .8 & 1.8 \\
\hline Total ............ & 100 & 100 & 100 & $100^{.1}$ & $100^{\circ}$ & 100 \\
\hline 2007 Survey of Consumer Finances & & & & & & \\
\hline Wages .................................. & 79.9 & 79.9 & 77.8 & 72.4 & 46.2 & 64.5 \\
\hline Interest or dividends & .1 & .3 & $\begin{array}{r}.0 \\
.7\end{array}$ & $\begin{array}{r}1.9 \\
1.9\end{array}$ & 7.8 & 3.7 \\
\hline Business, farm, self-employment & 1.8 & 5.3 & 6.9 & 7.9 & 24.7 & 13.6 \\
\hline Capital gains $\ldots \ldots \ldots \ldots \ldots$ & .1 & .4 & 1.3 & 2.9 & 14.4 & 6.7 \\
\hline Social Security or retirement & 9.5 & 10.9 & 11.8 & 14.1 & $\begin{array}{r}14.4 \\
6.2\end{array}$ & 9.6 \\
\hline Transfers or other ........... & 8.6 & $\begin{array}{r}10.9 \\
3.2\end{array}$ & $\begin{array}{l}11.8 \\
1.6\end{array}$ & $\begin{array}{r}14.1 \\
.8\end{array}$ & 0.2 & 1.9 \\
\hline Total & 100 & 100 & 100 & $100^{\circ}$ & 100 & 100 \\
\hline
\end{tabular}

$\dagger$ Less than 0.05 percent.

occupation have higher incomes than families in the three remaining occupation categories. Income is also higher for homeowners than for other families, and it is progressively higher for groups with greater net worth. ${ }^{7}$ Across the four regions of the country as defined by the Census Bureau, the ordering of median incomes over time has varied, but the means generally show higher values for the Northeast and the West than for the Midwest and the South. Finally, families living in metropolitan statistical areas (MSAs), which are relatively urban areas, have higher median and mean incomes than those living in rural areas. $^{8}$

\section{Income by Demographic Category}

Across the income distribution between 2004 and 2007 , only the second quintile and the top decile experienced substantial percentage changes in median income; the medians for both groups rose approximately 2 percent, though the dollar amount of the increase for the second quintile was only about $\$ 600$. $^{9}$ For other groups, changes in the median varied in direction, and in all instances they were less than 1 percent in absolute value. Similarly, the direction of changes in mean income was mixed, and the only substantial increase in dollar terms occurred for the top decile of the income distribution; the mean for that group rose almost 20 percent, more than twice the rate of change in the overall mean. Median

7. In this article, a family is treated as a homeowner if at least one person in the family owns at least some part of the family's primary residence.

8. For the Office of Management and Budget's definition of MSAs, see www.whitehouse.gov/omb/bulletins/fy2008/b08-01.pdf.

9. Selected percentiles of the income distribution for the past four surveys are provided in the appendix, along with definitions of selected subgroups of the distribution. income measured in the survey had been relatively flat for all income groups since 2001 after an earlier period of growth before 1998. Over this longer period, the rise in the mean was greatest for the top decile of the income distribution despite a dip for this group between 2001 and 2004. For the rest of the distribution, the increase of the mean more closely resembled that of the median.

Substantial proportional gains or losses in median income occurred across all age groups in the recent three-year period. The median declined for the age groups between 45 and 64 and for the 75-or-more age group, while it rose for the rest. For the 75-or-more age group, the decline was 12.3 percent. Since 1998, the age groups between 55 and 74 experienced the largest proportional rises in the median. In contrast to the recent changes in the median, the mean rose for all groups but especially for the 45-to-54 age group (8.5 percent) and the 65-to-74 age group (40.9 percent); these groups had experienced a decline in the mean between 2001 and 2004 .

By family structure, median incomes declined over the 2004-07 period for all groups except childless single families (those headed by a person who was neither married nor living with a partner); median income rose the most (5.1 percent) for childless families headed by a person aged 55 or older. The largest decline (4.5 percent) was for couples (families in which the family head was either married or living with a partner) with children. In contrast, mean income rose for all types of families except childless single families headed by a person aged 55 or older, for whom it fell 2.9 percent. Mean income rose the most (20.7 percent) for single families with children.

Across education groups, median incomes rose only for families headed by a person with less than a high school diploma and for families headed by a 
person with only some college education (who attended college but did not receive a degree); the increase of median income was relatively strong for the former group - 4.2 percent-but that group still had the lowest median income of all education groups. Mean incomes rose substantially for all education groups after declines in the preceding threeyear period. The increases were particularly pronounced for the groups with families headed by a person with only some college education (10.6 percent) or by a person with a college degree (11.4 percent).

In the 2004-07 period, the median income for white non-Hispanic families fell 4.6 percent, and the mean rose 9.4 percent. In contrast, the median for nonwhite or Hispanic families rose 12.5 percent, and the mean rose 8.7 percent. However, both the median and the mean values for nonwhites or Hispanics were substantially lower than the corresponding figures for non-Hispanic whites. Since 1998, the total gain in median income for nonwhite or Hispanic families was 23.9 percent, whereas it was 6.6 percent for other families; the gain in the mean over this period was larger and more similar for the two groups-27.0 percent for nonwhite or Hispanic families and 28.5 percent for other families. ${ }^{10}$

Median income rose from 2004 to 2007 for families headed by a person who was working for someone else (a rise of 4.6 percent) or was self-employed (a rise of 3.3 percent); the median fell for the retired group (7.8 percent) and the other-not-working group (9.7 percent). ${ }^{11}$ In contrast, the mean over this period rose for all groups except the other-not-working group, for which it fell 13.7 percent. Of the increases in the mean, the largest proportional change was the 23.3 percent rise for the self-employed group-the group with the highest levels of median and mean income by far. Over the previous three years, median

10. As noted in the appendix, the questions underlying the definition of race or ethnicity changed in earlier surveys. When restrictions are placed on the definition of the variable for racial and ethnic classification used in the tables in the article to make the series more comparable over time, the estimates change only slightly.

11. To be included in the retired group, the family head must report being retired and not currently working at any job or report being out of the labor force and over the age of 65. The other-not-working group comprises family heads who are unemployed and those who are out of the labor force but are neither retired nor over age 65; the composition of this group shifted from 2004 to 2007 to include fewer families with a head who had a college degree, thereby reversing a change seen between 2001 and 2004. In 2007, 66.9 percent of the other-notworking group was unemployed, and the remainder was out of the labor force; in 2004, 62.2 percent of the group was unemployed (data not shown in the tables). incomes had risen only for the retired and other-notworking groups, and the mean had risen only for the retired group.

Across occupation groups, median income rose moderately for families headed by a person working in a technical, sales, or service job (an increase of 7.5 percent), and it fell strongly for families headed by a person who was not working (a decline of 9.2 percent). For the other-occupation group, a group that predominantly comprises workers in traditional blue-collar occupations, the median was barely changed. In contrast, mean income rose for all groups, particularly for families headed by a person in a managerial or professional position (an increase of 10.8 percent) and for those headed by a person in a technical, sales, or service position (an increase of 16.0 percent), the groups with the highest mean incomes in 2007. Since 1998, the only substantial changes in the median were the increases for the managerial or professional group and for the technical, sales, or service group. The means for the groups showed a general pattern of increase over the period since 1998.

By region, median family incomes in the Northeast and the West converged from different directions to about the same value in 2007, and the medians in the Midwest and the South similarly converged. The median increased between 2004 and 2007 for families living in the South and the West, and it fell for others. The 8.1 percent decline for families in the Northeast offset only about one-half of a steep increase between 2001 and 2004. The rise for the West continued the only uninterrupted trend in the median across regions for the period shown. Declines in the median income in the Midwest since 2001 erased most of the substantial gains between 1998 and 2001. In 2007, mean income was highest in the Northeast, followed by the West. In 2001, the two had been closer, but growth flattened out for the West, while it continued for the Northeast. The mean incomes in the Midwest and the South have been comparable with one another since 1998, though the mean for the South increased strongly over the recent period while the mean for the Midwest fell back slightly since 2001.

In the recent three-year period, families in MSAs saw a 0.8 percent decline in median income, while those living in other areas saw a rise of 9.8 percent. Mean income has shown a general rise for both groups since 1998 .

By housing status, median and mean incomes rose both for homeowners and for other families from 2004 to 2007. All the increases were modest except 
the 10.0 percent increase in the mean for homeowners. As noted later in this article, homeownership declined slightly in the recent three-year period after rising for a number of years. Thus, changes in the composition of the group are likely to be smaller than in earlier years. Nonetheless, such changes were sufficient to cause the change in the median for both groups to be positive at the same time that the change in the overall median was negative.

By percentile of net worth, median income rose more than 1 percent over the recent three-year period only for the lowest quartile, for which the median increased 4.4 percent; the median declined somewhat for the third quartile and for the group between the 75th and 90th percentiles. ${ }^{12}$ The mean increased over the period for the lowest quartile (an increase of 6.2 percent), but it rose much more strongly ( 23.5 percent) for the top decile. Over the earlier years shown in the table, the most dramatic cumulative gains in the median were clearly for the top two groups. The mean rose at least somewhat for all groups, but the change was largest by far for the wealthiest 10 percent.

\section{Income Variability}

For a given family, income at a particular time may not be indicative of its "usual" income. Unemployment, a bonus, a capital loss or gain, or other factors may cause income to deviate temporarily from the usual amount. Although the SCF is a cross-sectional survey, it does provide some information on income variability. In 2007, 23.7 percent of families reported that their income for the preceding year was unusual9.2 percent reported it was unusually high, and 14.5 percent reported it was unusually low (data not shown in the tables). For those reporting unusual income, the median deviation of actual income from the usual amount was negative 17.3 percent of the normal level. A larger fraction of families in 2004 reported that their income was unusual-8.7 percent reported it was unusually high, and 19.8 percent reported it was unusually low.

Although a family's income may vary, such variability may be a well-recognized part of its financial planning. In 2007, 31.4 percent of families reported that they did not have a good idea of what their income would be for the next year, and 27.2 percent reported that they do not even usually have a good idea of their next year's income. The figures for 2004 were similar.

12. Selected percentiles of the distribution of net worth for the past four surveys are provided in the appendix.

\section{Saving}

Because saving out of current income is an important determinant of family net worth, the SCF asks respondents whether, over the preceding year, the family's spending was less than, more than, or about equal to its income. Though only qualitative, the answers are a useful indicator of whether families are saving. Asking instead for a specific dollar amount would require much more time from respondents and would likely lower the rate of response to the survey.

Overall, from 2004 to 2007, the proportion of families that reported that they had saved in the preceding year was about unchanged at 56.5 percent, a bit higher than the level in 1998 but still lower than the 2001 level. The general pattern of changes across demographic groups in the recent three-year period is one of small shifts. The previous survey had shown a broad pattern of declines.

Estimates of the personal saving rate from the national income and product accounts (NIPA) show an annual saving rate of less than 1 percent over the 2004-07 period. However, the SCF and NIPA concepts of saving differ in some important ways. First, the underlying SCF question asks only whether the family's spending has been less than, more than, or about the same as its income over the past year. Thus, families may be saving, but those that are doing so may be saving a relatively small amount; those that are spending more than their incomes may be spending a relatively large amount. Second, the NIPA measure of saving relies on definitions of income and consumption that may not be the same as those that respondents had in mind when answering the survey questions. For example, the NIPA measure of personal income includes payments employers make to their employees' defined-benefit pension plans but not the payments made from such plans to families, whereas the SCF measure includes only the latter. The SCF measure also includes realized capital gains, whereas the NIPA measure excludes capital gains of all forms, realized and unrealized.

A separate question in the survey asks about families' more typical saving habits. In 2007, 6.0 percent of families reported that their spending usually exceeds their income; 16.1 percent reported that the two are usually about the same; 35.7 percent reported that they typically save income "left over" at the end of the year, income of one family member, or unusual additional income; and 42.2 percent reported that they save regularly (data not shown in the tables). The fact that these figures are not much changed over the last three surveys suggests that variations in economic 
3. Reasons respondents gave as most important for their families' saving, distributed by type of reason, 19982007 surveys

Percent

\begin{tabular}{|c|c|c|c|c|}
\hline Type of reason & 1998 & 2001 & 2004 & 2007 \\
\hline Education .... & 11.0 & 10.9 & 11.6 & $8.4-4$ \\
\hline For the family .... & 4.1 & 5.1 & 4.7 & 5.5 \\
\hline Buying own home & 4.4 & 4.2 & 5.0 & 4.2 \\
\hline Purchases .......... & 9.7 & 9.5 & 7.7 & 10.0 \\
\hline Retirement .......... & 33.0 & 32.1 & 34.7 & 33. \\
\hline Liquidity .... & 29.8 & 31.2 & 30.0 & 32.0 \\
\hline Investments & 2.0 & 1.0 & 1.5 & $1.6(\mathrm{r})$ \\
\hline No particular reason ........ & 1.3 & 1.1 & .7 & 1.1 \\
\hline $\begin{array}{l}\text { When asked for a reason, } \\
\text { reported do not save }\end{array}$ & 4.9 & 4.9 & 4.0 & 3.3 \\
\hline Total $\ldots \ldots$ & 100 & 100 & 100 & 100 \\
\hline
\end{tabular}

NotE: See note to table 1 and text note 13 .

conditions over this period have had little effect on the longer-run saving plans of families.

The SCF also collects information on families' most important motivations for saving (table 3 )..$^{13}$ In 2007 , the most frequently reported motive was retirement related (33.9 percent of families), and the next most frequently reported was liquidity related (32.0 percent of families), a response that is generally taken to be indicative of saving for precautionary reasons. ${ }^{14}$ At least since 1998, these have been the dominant reported reasons, but saving for retirement has increased in importance. The education-related motive also appears to be important but less so in the latest survey; in 2007, 8.4 percent of families reported it as their primary motive, down 3.2 percentage points from 2004. The importance of saving for purchases rose 2.3 percentage points in 2007 after falling since before the 1998 survey in its prevalence as a reported motive for saving.

The survey asks families to estimate the amount of savings they need for emergencies and other unexpected contingencies, a measure of desired savings for precautionary purposes. ${ }^{15}$ The desired amount increases with income, but the amount is a lower percentage of usual income for higher levels of such income than for lower levels (table 3.1).

\section{NET WORTH}

From 2004 to 2007, inflation-adjusted net worth (wealth) - the difference between families' gross as-

13. Although families were asked to report their motives for saving regardless of whether they were currently saving, some families reported only that they do not save. The analysis here is confined to the first reason reported by families.

14. Liquidity-related reasons include "emergencies," the possibilities of unemployment and illness, and the need for ready money.

15. For an extended analysis of precautionary saving as measured in the SCF, see Arthur B. Kennickell and Annamaria Lusardi (2004), "Disentangling the Importance of the Precautionary Saving Motive," NBER Working Paper Series 10888 (Cambridge, Mass.: National Bureau of Economic Research, November).
3.1 .

\begin{tabular}{|c|c|c|}
\hline Family characteristic & $\begin{array}{l}\text { Median of desired } \\
\text { precautionary saving } \\
\text { (2007 dollars) }\end{array}$ & $\begin{array}{l}\text { Median of ratio } \\
\text { of desired amount } \\
\text { to usual income } \\
\text { (percent) }\end{array}$ \\
\hline All families . & 5,000 & 9.2 \\
\hline 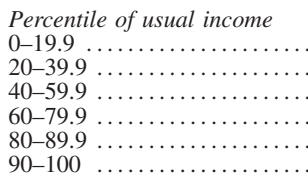 & $\begin{array}{r}2,000 \\
3,000 \\
5,000 \\
5,000 \\
10,000 \\
20,000\end{array}$ & $\begin{array}{r}14.0 \\
9.7 \\
9.4 \\
7.6 \\
8.1 \\
8.8\end{array}$ \\
\hline
\end{tabular}

sets and their liabilities-rose strongly, both in terms of the median and the mean (table 4). The median rose 17.7 percent, and the mean rose 13.0 percent; the corresponding values for the period from 2001 to 2004 were 1.0 percent and 6.0 percent. Both the median and the mean have risen consistently over the period since 1998, but overall the mean has gained more-54.7 percent, compared with a 31.8 percent increase in the median.

Movements in the dollar value of families' net worth are, by definition, a result of changes in investment, valuation, and patterns of ownership of financial assets (tables 5, 6, and 7) and nonfinancial assets (tables 8, 9, and 10), as well as decisions about acquiring or paying down debt (tables 11 through 18). A variety of financial decisions underlie these changes. The box "Shopping for Financial Services" provides a discussion of the intensity of families' decisionmaking efforts and their sources of financial information.

After the end of 2007, house prices continued to decline, and equity prices fell sharply. Although the survey cannot provide direct results about the overall effects of these and other such changes, it can provide some indication of the implications for families' finances. For this purpose, the value of assets invested directly or indirectly in publicly traded equity, the value of privately held businesses, and the net value of nonresidential real estate are assumed to have fallen at the overall rate of the Wilshire 5000 index from the time of the interview until October 2008. In addition, the value of residential properties-both primary residences and other residential real estateare assumed to have fallen in line with LoanPerformance Home Price Indexes from the time of the interview until October 2008. ${ }^{16}$ Changes are assumed to have affected all holders proportionately, and families are assumed to have made no changes in their holdings of these assets or any other assets or liabili-

16. Values of primary residences are adjusted by the state-level index. For other residential real estate, the geographic location is not reported in the SCF; thus, the national-level index is used to adjust values of these properties. The LoanPerformance Home Price Indexes are not seasonally adjusted. 
4. Family net worth, by selected characteristics of families, 1998-2007 surveys

Thousands of 2007 dollars

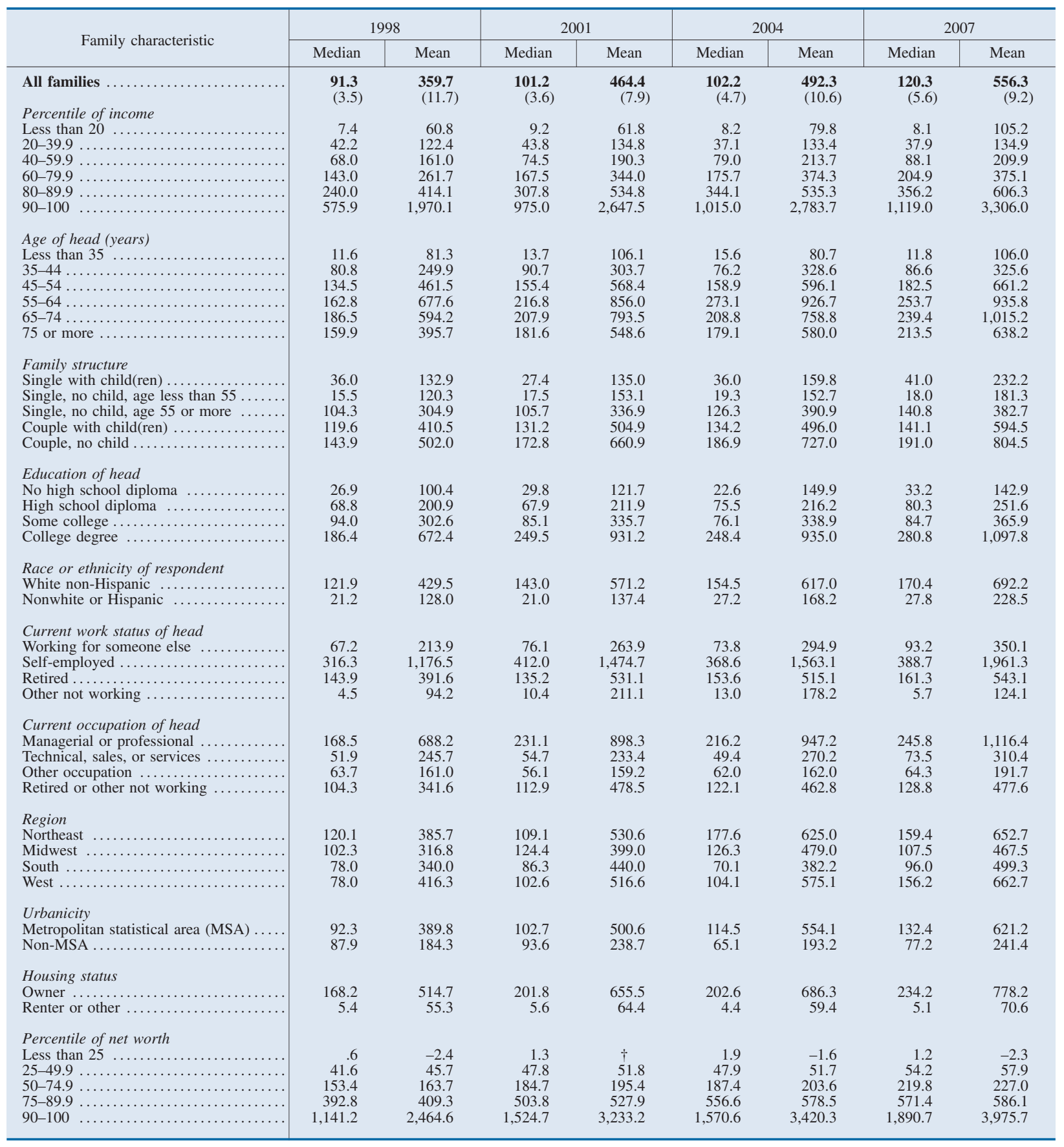

Note: See note to table 1.

$\dagger$ Less than 0.05 (\$50). 


\section{Shopping for Financial Services}

As a normal part of their financial lives, families must make a variety of decisions to select particular investments for any savings they may have, as well as to select the forms and terms of credit they may use. To the extent that families devote more or less attention to such activities or that they are better or worse informed, the wealth of otherwise comparable families may differ substantially over time.

The Survey of Consumer Finances (SCF) contains a self-assessment of families' intensity of shopping for borrowing or investing services. In 2007, about 55 percent of families reported that they undertake a moderate amount of shopping for either of these types of financial services (table A). ${ }^{1}$ Only about one-fourth of families

A. Intensity of shopping for borrowing or investing, 2007

Percent

\begin{tabular}{c|c|c}
\hline \multirow{2}{*}{ Intensity of shopping } & \multicolumn{2}{|c}{ Type of service } \\
\cline { 2 - 3 } & Borrowing & Investing \\
\hline Almost none $\ldots \ldots \ldots \ldots \ldots \ldots \ldots$ & 20.6 & 25.4 \\
Moderate amount $\ldots \ldots \ldots \ldots \ldots \ldots$ & 54.8 & 54.6 \\
A great deal $\ldots \ldots \ldots \ldots \ldots \ldots \ldots$ & 24.6 & 20.1 \\
\hline
\end{tabular}

reported shopping a great deal for loan terms, and only about one-fifth reported shopping a great deal for the best terms on investments. Even though the survey question is

1. The underlying question allows the survey respondent to shade the intermediate response toward a greater or lesser amount of shopping. About one-third of the respondents choose to do so, and of those, somewhat more than one-half shaded their response toward a greater degree of shopping. intended to elicit a description of behavior in general, the behavior reported could still be more reflective of the short-term needs for such services and consequently the immediate need for shopping. When broken out by categories of net worth, the patterns are very similar for all families for loan shopping (data not shown in the tables). For investment shopping, the data show a more pronounced gradient toward more intensive shopping by families with higher levels of wealth.

More families turn to friends, family members, or associates for financial information than to any other source of information on borrowing or investing (table B). This result suggests that there may be important feedback effects in financial outcomes; that is, families

B. Information used for decisions about borrowing or investing, 2007

Percent

\begin{tabular}{|c|c|c|}
\hline \multirow{2}{*}{ Source } & \multicolumn{2}{|c|}{ Type of service } \\
\hline & Borrowing & Investing \\
\hline Calling around $\ldots \ldots \ldots \ldots$ & 33.4 & 18.0 \\
\hline Magazines, newspapers, and & 197 & 17.5 \\
\hline Material in the mail ......... & 35.9 & 21.5 \\
\hline Internet $\ldots \ldots \ldots \ldots \ldots \ldots$ & 38.4 & 28.3 \\
\hline Friends, relatives, associates & 46.0 & 42.3 \\
\hline $\begin{array}{l}\text { Bankers, brokers, and other } \\
\text { sellers of financial services }\end{array}$ & 38.6 & 38.3 \\
\hline $\begin{array}{l}\text { Lawyers, accountants, and other } \\
\quad \text { financial advisors } \ldots . . \ldots \ldots \ldots . .\end{array}$ & 19.5 & 29.3 \\
\hline Does not borrow or invest ........ & 9.5 & 9.9 \\
\hline
\end{tabular}

NoTE: Figures sum to more than 100 because of reporting of multiple sources. ties. Taken together, these assumptions imply large drops in median and mean net worth since the 2007 survey-17.8 percent and 22.7 percent, respectively. Relative to the values in the 2004 SCF, adjusted median net worth is 3.2 percent lower, and the adjusted mean is 12.7 percent lower. ${ }^{17}$

By age group, median and mean net worth show a "hump" pattern that generally peaks in the 55-to-64

17. Most of the projected decline in the median is a result of the adjustments to primary residences and publicly traded equity; if only the values of primary residences and of directly or indirectly held equity are adjusted, median net worth as of October 2008 declines 15.0 percent relative to the level observed in the 2007 survey. In contrast, the corresponding mean of the data under the more limited adjustment is only 12.0 percent lower than the unadjusted value, or just more than one-half of the decline implied by the broader set of adjustments; this result reflects the fact that the value of businesses and real estate other than primary residences is relatively concentrated among wealthier families. age group. This pattern reflects both life-cycle saving behavior and a historical pattern of long-run growth in inflation-adjusted wages. The median and mean values of wealth rise in tandem with income, a relationship reflecting both income earned from assets and a higher likelihood of saving among higherincome families. Wealth shows strong differentials across groups defined in terms of family structure, education, racial or ethnic background, work status, occupation, housing status, and the urbanicity and region of residence; these differentials generally mirror those for income, but the wealth differences are larger.

\section{Net Worth by Demographic Category}

Analysis by demographic group for the 2004-07 period shows a pattern of gains of varying sizes in 
who know relatively well-informed people may obtain better services. Sellers of financial services-bankers, brokers, and so on-are the second most frequently cited source of information for borrowing or investing. The Internet was reported by 38.4 percent of families as a source for information on borrowing and by 28.3 percent for information on investing. Although the Internet, in principle, makes an enormous amount of information available to a family, interpretation of the information may still be an important consideration. However, the proliferation of financial planning tools may mitigate this concern. When viewed across categories of net worth, the data show similar patterns of use of sources of information by all groups (data not shown in the tables).

In addition to serving as a source of information, the Internet can also be a medium for obtaining financial services. In 2007, 49.4 percent of families reported using the Internet to access at least some type of service at one of the financial institutions they used (data not shown in the tables). If accessing information and using services are combined, the Internet played a part in the financial life of 59.7 percent of all families (table $\mathrm{C}$ ). This figure is up sharply from 46.5 percent in 2004 and 32.5 percent in 2001. The proportion of such users rises strongly over net worth groups: Among the least wealthy 25 percent of families, 50.3 percent made such use of the Internet, whereas the figure was 75.6 percent for the wealthiest 10 percent (data not shown in the tables). More striking is the variation over age groups. Among families headed by a person younger than $35,71.9$ percent reported using the
C. Use of the Internet for financial information or financial services, by age of head, 2007

Percent

\begin{tabular}{|c|c|}
\hline Family characteristic & Percentages of families \\
\hline All families . & 59.7 \\
\hline $\begin{array}{l}\text { Age of head (years) } \\
\text { Less than } 35 \ldots \ldots \ldots \\
35-44 \ldots \ldots \ldots \ldots \ldots \\
45-54 \ldots \ldots \ldots \ldots \ldots \\
55-64 \ldots \ldots \ldots \ldots \ldots \\
65-74 \ldots \ldots \ldots \ldots \ldots \\
75 \text { or more } \ldots \ldots \ldots \ldots\end{array}$ & $\begin{array}{l}71.9 \\
70.8 \\
69.1 \\
59.1 \\
40.3 \\
16.5\end{array}$ \\
\hline $\begin{array}{l}\text { MEMO } \\
\text { All families, } 2004 \\
\text { All families, } 2001 \text {. }\end{array}$ & $\begin{array}{l}46.5 \\
32.5\end{array}$ \\
\hline
\end{tabular}

Internet for financial information or services, whereas the figure for families with a head aged 75 or older was only 16.5 percent. If the relatively greater expression of such behavior by younger families persists as they age, and if succeeding cohorts follow their example, Internet-based financial services may become even more important in the future. ${ }^{2}$

2. For a discussion of the definition of local banking markets, see Dean F. Amel, Arthur B. Kennickell, and Kevin B. Moore (2008), "Banking Market Definition: Evidence from the Survey of Consumer Finances," Finance and Economics Discussion Series 2008-35 (Washington: Board of Governors of the Federal Reserve System, October), www.federalreserve.gov/pubs/feds/2008/200835/200835pap.pdf. median and mean net worth for most groups. But a small number of groups experienced losses, and some had noticeably different shifts in their median and mean net worth.

Median net worth rose for all percentile groups of the distribution of net worth except for families in the lowest quartile. In that group, the median fell from $\$ 1,900$ to $\$ 1,200$; the mean fell from negative $\$ 1,600$ in 2004 to negative $\$ 2,300$ in 2007 . For the rest of the distribution of net worth, the median and mean over the recent three-year period rose substantially for all other groups except the 75th-to-90th percentile group, which had seen relatively large gains over the preceding three years. Gains for the top wealth group were unbroken back to at least 1998.

Over the recent period, median net worth increased for all income groups above the 20th percentile and especially for families in the fourth quintile, for which the median rose 16.6 percent; the mean for this group was little changed. Families in the lowest income quintile had the largest proportional increase in the mean-31.8 percent - a rise due, in part, to an increase in the fraction of the group consisting of relatively wealthy families with incomes that are likely to have been temporarily low (data not shown in the tables). The mean rose for the other income groups, and it rose most for the highest decile group-an 18.8 percent gain. Over the preceding years shown, median net worth had increased for all groups except the second income quintile; the mean had risen for all income groups.

The survey shows some substantial movements of net worth by age group between 2004 and 2007 . Median net worth rose most strongly-19.2 percentfor the 75-or-more age group, which had seen relatively modest change over the previous three-year 
period. The less-than-35 age group saw a large decline in the median-24.4 percent-over the more recent period; at the same time, median wealth fell 7.1 percent for the 55-to-64 age group. Mean wealth rose just more than 10 percent for families in the 45-to-54 and 75-or-more age groups, and it increased more than 30 percent for families in the less-than-35 and 65-to-74 age groups; mean wealth declined, however, for the 35-to-44 group and was about unchanged for the 55-to-64 group. Many of the changes observed contrast in size or direction with the changes in the preceding three-year period.

By family structure, single families with children had the largest increases from 2004 to 2007 in both median and mean net worth-13.9 percent and 45.3 percent, respectively_-but these families had the second-lowest level of net worth (after younger single families without children). Median net worth increased for all family-structure groups except younger single families without children, and the mean increased for all except older single families without children.

From 2004 to 2007, median net worth increased for all education groups. The change was particularly large - 46.9 percent—for the no-high-school-diploma group. At the same time, this group was the only one that did not see a rise in mean net worth; its mean declined 4.7 percent. The shifts for this group were the opposite of the pattern in the preceding three-year period, during which the median fell and the mean rose.

The data show gains from 2004 to 2007 in median and mean wealth for both categories of race or ethnicity. Gains in the median and the mean were roughly the same for white non-Hispanic families10.3 percent and 12.2 percent, respectively. But for nonwhite or Hispanic families, the change in the median-2.2 percent-was far smaller than that in the mean-35.9 percent. ${ }^{18}$ In the preceding three-year period, both the median and the mean for nonwhites or Hispanics had risen more strongly than those for other families. Despite some continuing signs of convergence, in 2007, the median and mean of net worth for white non-Hispanic families remained much higher than those for nonwhite or Hispanic families. In contrast to the whole group of nonwhite or Hispanic families, the subgroup of African American families saw a 24.1 percent decline in their

18. If the additional information on Hispanic or Latino ethnic identification available in the SCF is used in the classification of the 2007 results, the median net worth of nonwhites or Hispanics was $\$ 31,000$, and the mean was $\$ 237,900$; for other families, the median was $\$ 174,100$, and the mean was $\$ 701,800$. These figures are all slightly higher than the corresponding values reported in table 4 . median net worth from $2004(\$ 22,400)$ to 2007 $(\$ 17,000)$, but their mean net worth rose 9.3 percent, from $\$ 121,500$ to $\$ 132,800$; over the $2001-04$ period, the median for the group had shown virtually no change, while the mean had risen 36.4 percent (data not shown in the tables).

Among work-status groups, median and mean net worth rose from 2004 to 2007 for all families except those headed by persons who were not working for reasons other than retirement (the other-not-working group), which showed substantial declines in both measures. The group had the lowest levels of both median and mean net worth of all work-status groups. Although the dollar amounts of the changes in median and mean net worth for the self-employed group were far larger than those for the other groups over the period from 1998 to 2007, the percentage increase in the median for the self-employed group was below the rates for all other work-status categories except the retired group. The percentage increase in the mean for the self-employed group was just slightly higher than that for the working-for-someone-else group.

Median and mean net worth increased for all occupation groups in the recent three-year period, but they did so most markedly for families headed by a worker in a technical, sales, or service occupation or by a worker in a managerial or professional occupation. Over the period since 1998, the median for families in the residual other-occupation category barely rose, and the increase in the mean was the smallest of any occupation group. All other groups had greater than a 20 percent increase in their median and mean net worth over this period.

Between 2004 and 2007, median net worth fell for families living in the Northeast or the Midwest, while it rose strongly for those in the South or the West. Mean net worth for families in the Northeast or the Midwest also lagged behind that for families in the other regions. Over the longer period from 1998 to 2007, median and mean net worth moved up most strongly in the Northeast and the West; these regions ended the period with quite similar medians and means. The Midwest and the South also ended the period with fairly similar values, at levels considerably below those for the Northeast and the West.

By urbanicity of the place of residence, in the recent three-year period, median net worth increased by about the same proportion in MSA and non-MSA areas, but the mean advanced by a much larger proportion in non-MSA areas. However, over the longer period since 1998, median and mean wealth rose more rapidly for MSAs, and in 2007 both the 
median and mean net worth for families in MSAs remained substantially above that for families in non-MSAs.

By housing status, the percentage increases in median net worth between 2004 and 2007 were very similar for both groups, and the increase in the mean for non-homeowners (hereafter, renters) was somewhat higher. From 1998 to 2007-a time of rising house prices, on balance- the increase in median and mean net worth for homeowners far outstripped that for renters.

\section{ASSETS}

At 97.7 percent in 2007, the overall proportion of families with any asset was barely changed from 2004 (first half of tables 9.A and 9.B, last column). Overall, this figure has risen 0.9 percentage point since 1998 (data not shown in the tables). Across demographic groups, the pattern of changes in the recent three-year period is mostly one of small increases or decreases. Noticeable exceptions are declines for the following groups: the lowest quintile of the income distribution (2.4 percentage points); single families with children (1.2 percentage points); younger single families without children (1.7 percentage points); families headed by a person whose work status was retired (1.6 percentage points) or who was in the related retired-or-other-not-working category (1.2 percentage points); families headed by a person aged 75 or older (1.5 percent); and families living in the Northeast (3.3 percentage points). ${ }^{19}$ For many groups, the figure remained at or near 100 percent.

From 2004 to 2007, median assets for families having any assets rose 16.6 percent, from $\$ 189,900$ to $\$ 221,500$ (second half of tables 9.A and 9.B, last column), and the mean rose 13.1 percent, from $\$ 591,300$ to $\$ 668,500$ (memo line). These percentage changes closely resemble those for overall net worth, but examination of changes in median assets by demographic groups reveals differences. Because changes in ownership were generally small, these differences must largely represent variations in the amount of borrowing. Across net worth groups, the percentage changes in median assets and net worth were most similar for families in the top quartile of the distribution of net worth; for all except the lowest quartile of that distribution, the changes were more roughly similar; and for the lowest quartile of the distribution, the percentage decline in assets was much larger than that for net worth. For white non-

19. The retired-or-other-not-working occupation category encompasses the retired and the other-not-working work-status categories.
5. Value of financial assets of all families, distributed by type of asset, 1998-2007 surveys

Percent

\begin{tabular}{|c|c|c|c|c|}
\hline Type of financial asset & 1998 & 2001 & 2004 & 2007 \\
\hline Transaction accounts ... & 11.4 & 11.4 & 13.2 & 11.0 \\
\hline Certificates of deposit ... & 4.3 & 3.1 & 3.7 & 4.1 \\
\hline Savings bonds ........ & .7 & .7 & .5 & .4 \\
\hline Bonds ......... & 4.3 & 4.5 & 5.3 & 4.2 \\
\hline Stocks $\ldots \ldots \ldots \ldots \ldots \ldots \ldots$ & 22.7 & 21.5 & 17.6 & 17.9 \\
\hline Pooled investment funds (excluding & & & & \\
\hline money market funds) $\ldots \ldots \ldots$ & 12.4 & 12.1 & 14.7 & 15.9 \\
\hline Retirement accounts ...... & 27.6 & 28.9 & 32.0 & 34.6 \\
\hline Cash value life insurance & 6.4 & 5.3 & 3.0 & 3.2 \\
\hline Other managed assets ..... & 8.6 & 10.5 & 8.0 & 6.5 \\
\hline Other .................... & 1.7 & 1.9 & 2.1 & 2.1 \\
\hline Total & 100 & 100 & 100 & 100 \\
\hline MEMO & & & & \\
\hline $\begin{array}{l}\text { Financial assets as a share } \\
\text { of total assets ........ }\end{array}$ & 40.7 & 42.2 & 35.7 & 33.9 \\
\hline
\end{tabular}

NotE: For this and following tables, see text for definition of asset categories. Also see note to table 1.

Hispanic families, median assets rose 9.9 percent, while median net worth rose 10.3 percent; but for nonwhites or Hispanics, median assets rose 36.4 percent, and median net worth rose only 2.2 percent. For homeowners, median assets increased 8.1 percent, but median net worth increased 15.6 percent; for renters, median assets barely changed, but median net worth rose 15.9 percent. Percentage changes in the medians of assets and net worth were similar across region and urbanicity of the place of residence. Over the preceding three-year period, median assets had risen 9.8 percent and mean assets had risen 8.3 percent, compared with corresponding figures for net worth of 1.0 percent and 6.0 percent.

\section{Financial Assets}

Although the level of financial assets rose from 2004 to 2007, financial assets as a share of total assets fell 1.8 percentage points, to 33.9 percent (table 5 , memo line); this movement continues a decline in this share from a level in 2001 (42.2 percent) that marked the high point observed in the survey since at least 1989. The relative shares of various financial assets also shifted. Declines in the percentage shares of transaction accounts, bonds, and "other managed assets" were mostly offset by increases in the shares of retirement accounts and pooled investment funds. ${ }^{20}$ After declines in the previous two surveys, the share of assets attributable to publicly traded stocks held directly by families edged up.

Overall, the rate of ownership of any financial asset was virtually unchanged over the recent survey

20. The definitions of asset categories in table 5 are given later in the article, in the sections of text devoted to those categories. 
6. Family holdings of financial assets, by selected characteristics of families and type of asset, 2004 and 2007 surveys

A. 2004 Survey of Consumer Finances

\begin{tabular}{|c|c|c|c|c|c|c|c|c|c|c|c|}
\hline Family characteristic & $\begin{array}{c}\text { Trans- } \\
\text { action } \\
\text { accounts }\end{array}$ & $\begin{array}{l}\text { Certifi- } \\
\text { cates of } \\
\text { deposit }\end{array}$ & $\begin{array}{c}\text { Savings } \\
\text { bonds }\end{array}$ & Bonds & Stocks & $\begin{array}{c}\text { Pooled } \\
\text { invest- } \\
\text { ment } \\
\text { funds }\end{array}$ & $\begin{array}{c}\text { Retire- } \\
\text { ment } \\
\text { accounts }\end{array}$ & $\begin{array}{c}\text { Cash } \\
\text { value life } \\
\text { insurance }\end{array}$ & $\begin{array}{c}\text { Other } \\
\text { managed } \\
\text { assets }\end{array}$ & Other & $\begin{array}{l}\text { Any } \\
\text { financial } \\
\text { asset }\end{array}$ \\
\hline & \multicolumn{11}{|c|}{ Percentage of families holding asset } \\
\hline All families & 91.3 & 12.7 & 17.6 & 1.8 & 20.7 & 15.0 & 49.7 & 24.2 & 7.3 & 10.0 & 93.8 \\
\hline Percentile of income & & & & & & & & & & & \\
\hline $\begin{array}{l}\text { Less than } 20 \ldots \ldots \ldots \\
20-39.9 \ldots \ldots \ldots \ldots\end{array}$ & $\begin{array}{l}75.5 \\
87.3\end{array}$ & $\begin{array}{r}5.0 \\
12.7\end{array}$ & $\begin{array}{l}6.2 \\
8.8\end{array}$ & $\begin{array}{l}* \\
*\end{array}$ & $\begin{array}{l}5.1 \\
8.2\end{array}$ & $\begin{array}{l}3.6 \\
7.6\end{array}$ & $\begin{array}{l}10.1 \\
29.8\end{array}$ & $\begin{array}{l}14.0 \\
19.0\end{array}$ & $\begin{array}{l}3.1 \\
4.9\end{array}$ & $\begin{array}{l}7.1 \\
99\end{array}$ & 80.1 \\
\hline $40-59.9$ & 95.9 & 11.8 & 15.4 & * & 16.4 & 12.7 & 53.5 & 24.4 & 7.9 & 9.3 & 98.5 \\
\hline $60-79.9$ & 98.4 & 15.0 & 26.5 & 2.1 & 28.1 & 18.6 & 69.7 & 29.7 & 7.8 & 11.2 & 99.1 \\
\hline $80-89.9$ & 99.1 & 16.3 & 32.3 & 2.9 & 35.9 & 26.2 & 81.9 & 29.6 & 12.2 & 11.4 & 99.8 \\
\hline $90-100$ & 100.0 & 21.5 & 29.9 & 8.9 & 55.0 & 39.1 & 88.5 & 38.1 & 13.0 & 13.4 & 100.0 \\
\hline Age of head (years) & & & & & & & & & & & \\
\hline Less than $35 \ldots \ldots$. & 86.4 & 5.6 & 15.3 & * & 13.3 & 8.3 & 40.2 & 11.0 & 2.9 & 11.6 & 90.1 \\
\hline $35-44 \ldots \ldots \ldots$ & 90.8 & 6.7 & 23.3 & .6 & 18.5 & 12.3 & 55.9 & 20.1 & 3.7 & 10.0 & 93.6 \\
\hline $45-54 \ldots \ldots$ & 91.8 & 11.9 & 21.0 & 1.8 & 23.2 & 18.2 & 57.7 & 26.0 & 6.2 & 12.1 & 93.6 \\
\hline $55-64$. & 93.2 & 18.1 & 15.2 & 3.3 & 29.1 & 20.6 & 62.9 & 32.1 & 9.4 & 7.2 & 95.2 \\
\hline $65-74 \ldots \ldots$ & 93.9 & 19.9 & 14.9 & 4.3 & 25.4 & 18.6 & 43.2 & 34.8 & 12.8 & 8.1 & 96.5 \\
\hline 75 or more & 96.4 & 25.7 & 11.0 & 3.0 & 18.4 & 16.6 & 29.2 & 34.0 & 16.7 & 8.1 & 97.6 \\
\hline \multicolumn{12}{|l|}{ Family structure } \\
\hline Single with child(ren) $\ldots \ldots \ldots \ldots$ & 87.2 & 8.8 & 9.4 & * & 9.6 & 7.4 & 34.1 & 19.9 & 3.7 & 13.7 & 91.1 \\
\hline Single, no child, age less than 55 & 85.1 & 5.9 & 11.9 & .3 & 12.4 & 10.2 & 37.5 & 14.0 & 2.8 & 13.8 & 88.9 \\
\hline Single, no child, age 55 or more & 91.8 & 18.8 & 9.1 & 2.6 & 18.0 & 16.0 & 32.8 & 28.8 & 14.0 & 7.8 & 94.4 \\
\hline Couple with child(ren) $\ldots \ldots \ldots \ldots$ & 93.5 & 14.9 & 25.1 & .9 & 23.3 & 11.7 & 61.4 & 24.7 & 6.1 & 7.4 & 96.4 \\
\hline Couple, no child ....... & 94.0 & 13.6 & 22.1 & 2.7 & 26.2 & 19.0 & 59.8 & 27.7 & 7.9 & 9.1 & 95.5 \\
\hline \multicolumn{12}{|l|}{ Education of head } \\
\hline No high school diploma & 72.4 & 5.6 & 4.2 & * & 4.7 & 2.3 & 16.2 & 13.7 & 3.0 & 5.2 & 77.4 \\
\hline High school diploma ... & 89.1 & 12.9 & 14.2 & .4 & 12.4 & 9.2 & 43.6 & 23.0 & 5.4 & 8.4 & 92.9 \\
\hline Some college ......... & 94.3 & 9.4 & 19.3 & .6 & 17.7 & 12.6 & 47.7 & 23.8 & 6.2 & 14.4 & 96.6 \\
\hline College degree & 99.1 & 17.0 & 24.9 & 4.1 & 35.3 & 26.1 & 68.9 & 29.5 & 10.9 & 10.9 & 99.6 \\
\hline \multicolumn{12}{|l|}{ Race or ethnicity of respondent } \\
\hline Nonwhite or Hispanic . ............ & 80.6 & 6.0 & 8.5 & $*$ & $\begin{array}{r}25.3 \\
8.0\end{array}$ & $\begin{array}{r}18.9 \\
5.0\end{array}$ & $\begin{array}{l}30.1 \\
32.9\end{array}$ & $\begin{array}{l}20.8 \\
17.4\end{array}$ & 2.1 & $\begin{array}{l}10.2 \\
9.4\end{array}$ & 85.0 \\
\hline \multicolumn{12}{|l|}{ Current work status of head } \\
\hline Working for someone else .. & 92.2 & 9.8 & 20.1 & .8 & 19.6 & 13.5 & 57.1 & 21.8 & 5.4 & $\begin{array}{r}9.5 \\
15\end{array}$ & 94.5 \\
\hline Self-employed ............. & 94.4 & 14.2 & 18.7 & 4.3 & 31.6 & 22.3 & 54.6 & 29.8 & 7.6 & 15.1 & 96.1 \\
\hline Retired ................. & 90.4 & 20.2 & 11.4 & 3.5 & 19.0 & 16.2 & 32.9 & 29.7 & 12.8 & 8.4 & 93.6 \\
\hline Other not working & 76.2 & 7.9 & 14.5 & $*$ & 14.3 & 10.2 & 24.9 & 10.7 & $*$ & 11.5 & 79.6 \\
\hline \multicolumn{12}{|l|}{ Current occupation of head } \\
\hline Managerial or professional . & 98.5 & 14.8 & 25.5 & 3.1 & 32.9 & 24.3 & 68.5 & 27.5 & 8.2 & 13.2 & 99.5 \\
\hline Technical, sales, or services & 90.1 & 8.9 & 18.5 & .3 & 15.6 & 9.7 & 48.5 & 21.9 & 4.9 & 8.6 & 92.9 \\
\hline Other occupation ........... & 87.2 & 6.4 & 13.8 & $*$ & 13.0 & 8.1 & 49.7 & 18.7 & 3.3 & 8.5 & 90.4 \\
\hline Retired or other not working & 88.2 & 18.3 & 11.8 & 3.0 & 18.2 & 15.3 & 31.6 & 26.8 & 11.1 & 8.9 & 91.4 \\
\hline \multicolumn{12}{|l|}{ Region } \\
\hline Northeast & 94.6 & 15.3 & 21.5 & 1.9 & 27.8 & 18.8 & 57.0 & 24.6 & 7.7 & 8.6 & 96.4 \\
\hline Midwest . & 94.4 & 14.9 & 23.6 & 1.6 & 23.4 & 15.2 & 57.3 & 30.3 & 11.5 & 10.7 & 96.5 \\
\hline South .... & 86.9 & 11.7 & 12.7 & 2.0 & 15.4 & 12.6 & 41.6 & 24.1 & 4.7 & 9.5 & 90.7 \\
\hline West. & 92.6 & 9.7 & 16.1 & 1.7 & 20.4 & 15.6 & 48.9 & 17.5 & 6.7 & 11.0 & 94.0 \\
\hline \multicolumn{12}{|l|}{ Urbanicity } \\
\hline Metropolitan statisical area (MSA) ... & 91.6 & 12.3 & 18.4 & 2.0 & 22.6 & 16.4 & 51.8 & 24.6 & 7.8 & 10.4 & 93.9 \\
\hline Non-MSA ....................... & 90.0 & 14.6 & 14.0 & * & 11.0 & 8.5 & 39.5 & 22.3 & 4.8 & 7.9 & 93.2 \\
\hline \multicolumn{12}{|l|}{ Housing status } \\
\hline Owner .......... & 96.0 & 15.9 & 21.2 & 2.6 & 25.8 & 19.2 & 60.2 & 30.1 & 9.6 & 9.6 & 97.5 \\
\hline Renter or other & 80.9 & 5.6 & 9.5 & .2 & 9.1 & 5.7 & 26.2 & 11.0 & 2.0 & 10.9 & 85.5 \\
\hline \multicolumn{12}{|l|}{ Percentile of net worth } \\
\hline Less than $25 \ldots \ldots \ldots$. & 75.4 & 2.2 & 6.2 & $*$ & 3.6 & 2.0 & 14.3 & 7.7 & $*$ & 6.9 & 79.8 \\
\hline $25-49.9 \ldots \ldots \ldots \ldots \ldots$ & 92.0 & 6.5 & 13.2 & * & 9.3 & 7.2 & 43.1 & 19.3 & 2.3 & 9.5 & 96.1 \\
\hline $50-74.9$ & 98.0 & 16.0 & 22.7 & $*$ & 21.0 & 12.5 & 61.8 & 30.1 & 8.8 & 10.2 & 99.4 \\
\hline $75-89.9$ & 99.7 & 24.2 & 28.5 & 3.2 & 39.1 & 32.4 & 77.6 & 36.7 & 15.6 & 11.2 & 100.0 \\
\hline $90-100$ & 100.0 & 28.8 & 28.1 & 12.7 & 62.9 & 47.3 & 82.5 & 43.8 & 21.0 & 16.4 & 100.0 \\
\hline
\end{tabular}


6. Family holdings of financial assets, by selected characteristics of families and type of asset, 2004 and 2007 surveysContinued

A. 2004 Survey of Consumer Finances-continued

\begin{tabular}{|c|c|c|c|c|c|c|c|c|c|c|c|}
\hline Family characteristic & $\begin{array}{l}\text { Trans- } \\
\text { action } \\
\text { accounts }\end{array}$ & $\begin{array}{l}\text { Certifi- } \\
\text { cates of } \\
\text { deposit }\end{array}$ & $\begin{array}{l}\text { Savings } \\
\text { bonds }\end{array}$ & Bonds & Stocks & $\begin{array}{l}\text { Pooled } \\
\text { invest- } \\
\text { ment } \\
\text { funds }\end{array}$ & $\begin{array}{c}\text { Retire- } \\
\text { ment } \\
\text { accounts }\end{array}$ & $\begin{array}{c}\text { Cash } \\
\text { value life } \\
\text { insurance }\end{array}$ & $\begin{array}{c}\text { Other } \\
\text { managed } \\
\text { assets }\end{array}$ & Other & $\begin{array}{l}\text { Any } \\
\text { financial } \\
\text { asset }\end{array}$ \\
\hline & \multicolumn{11}{|c|}{ Median value of holdings for families holding asset (thousands of 2007 dollars) } \\
\hline All families & 4.1 & 16.5 & 1.1 & 71.4 & 16.5 & 44.4 & 38.7 & 6.6 & 49.4 & 4.4 & 25.3 \\
\hline 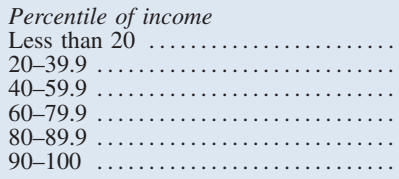 & $\begin{array}{r}.7 \\
1.6 \\
3.3 \\
7.1 \\
12.1 \\
30.8\end{array}$ & $\begin{array}{l}11.0 \\
15.4 \\
11.0 \\
19.8 \\
22.0 \\
36.2\end{array}$ & $\begin{array}{r}.4 \\
.7 \\
.9 \\
1.1 \\
.9 \\
2.2\end{array}$ & $\begin{array}{r}* \\
* \\
* \\
87.9 \\
38.4 \\
175.7\end{array}$ & $\begin{array}{r}6.6 \\
8.8 \\
13.2 \\
11.0 \\
16.5 \\
64.5\end{array}$ & $\begin{array}{r}16.8 \\
27.5 \\
25.3 \\
28.0 \\
36.8 \\
137.3\end{array}$ & $\begin{array}{r}5.5 \\
11.0 \\
19.0 \\
35.1 \\
76.9 \\
201.4\end{array}$ & $\begin{array}{r}3.1 \\
4.1 \\
5.5 \\
7.7 \\
11.0 \\
22.0\end{array}$ & $\begin{array}{r}24.1 \\
54.9 \\
39.5 \\
38.4 \\
54.9 \\
109.8\end{array}$ & $\begin{array}{r}2.7 \\
2.2 \\
2.7 \\
4.4 \\
5.5 \\
22.0\end{array}$ & $\begin{array}{r}1.5 \\
5.3 \\
17.0 \\
53.2 \\
119.1 \\
401.2\end{array}$ \\
\hline 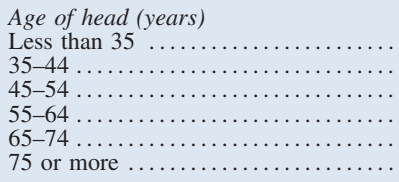 & $\begin{array}{l}2.0 \\
3.3 \\
5.3 \\
7.4 \\
6.0 \\
7.1\end{array}$ & $\begin{array}{r}4.4 \\
11.0 \\
12.1 \\
31.9 \\
22.0 \\
24.2\end{array}$ & $\begin{array}{r}.5 \\
.5 \\
1.1 \\
2.7 \\
3.3 \\
5.5\end{array}$ & $\begin{array}{r}* \\
11.0 \\
32.9 \\
87.9 \\
43.9 \\
324.0\end{array}$ & $\begin{array}{r}4.8 \\
11.0 \\
15.9 \\
27.5 \\
46.1 \\
54.9\end{array}$ & $\begin{array}{r}8.8 \\
17.5 \\
54.9 \\
82.4 \\
65.9 \\
65.9\end{array}$ & $\begin{array}{l}12.1 \\
30.6 \\
61.0 \\
91.2 \\
87.9 \\
32.9\end{array}$ & $\begin{array}{r}3.3 \\
5.5 \\
8.8 \\
11.0 \\
8.8 \\
5.5\end{array}$ & $\begin{array}{r}5.5 \\
20.1 \\
47.2 \\
71.4 \\
65.9 \\
54.9\end{array}$ & $\begin{array}{r}1.1 \\
3.8 \\
5.5 \\
7.7 \\
11.0 \\
24.2\end{array}$ & $\begin{array}{r}5.7 \\
20.9 \\
42.4 \\
85.7 \\
39.6 \\
42.6\end{array}$ \\
\hline $\begin{array}{l}\text { Family structure } \\
\text { Single with child(ren) } \ldots \ldots \ldots \ldots \ldots \\
\text { Single, no child, age less than } 55 \ldots \ldots \\
\text { Single, no child, age } 55 \text { or more } \ldots \ldots \\
\text { Couple with child(ren) } \ldots \ldots \ldots \ldots \ldots \ldots \\
\text { Couple, no child ....................... }\end{array}$ & $\begin{array}{l}1.4 \\
1.6 \\
3.3 \\
5.3 \\
6.7\end{array}$ & $\begin{array}{l}11.0 \\
11.0 \\
20.0 \\
11.0 \\
22.0\end{array}$ & $\begin{array}{r}.4 \\
1.1 \\
2.2 \\
.9 \\
1.1\end{array}$ & $\begin{array}{r}* \\
32.9 \\
68.1 \\
109.8 \\
87.9\end{array}$ & $\begin{array}{r}6.6 \\
8.8 \\
30.4 \\
6.7 \\
22.0\end{array}$ & $\begin{array}{l}23.1 \\
16.5 \\
68.6 \\
24.2 \\
54.9\end{array}$ & $\begin{array}{l}15.4 \\
15.4 \\
40.6 \\
39.0 \\
58.4\end{array}$ & $\begin{array}{r}2.2 \\
5.5 \\
3.5 \\
5.5 \\
11.0\end{array}$ & $\begin{array}{r}6.6 \\
32.9 \\
71.4 \\
32.9 \\
49.4\end{array}$ & $\begin{array}{r}3.3 \\
2.2 \\
11.0 \\
5.5 \\
6.6\end{array}$ & $\begin{array}{r}5.5 \\
6.0 \\
27.0 \\
32.4 \\
48.3\end{array}$ \\
\hline $\begin{array}{l}\text { Education of head } \\
\text { No high school diploma } \ldots \ldots \ldots \ldots \ldots \\
\text { High school diploma } \ldots \ldots \ldots \ldots \ldots \ldots \\
\text { Some college } \ldots \ldots \ldots \ldots \ldots \ldots \ldots \ldots \ldots \\
\text { College degree } \ldots \ldots \ldots \ldots \ldots \ldots \ldots\end{array}$ & $\begin{array}{r}1.2 \\
2.8 \\
2.9 \\
10.1\end{array}$ & $\begin{array}{l}16.5 \\
19.2 \\
11.0 \\
20.9\end{array}$ & $\begin{array}{r}.5 \\
.7 \\
.9 \\
1.1\end{array}$ & $\begin{array}{r}* \\
22.0 \\
168.6 \\
87.9\end{array}$ & $\begin{array}{r}8.2 \\
8.2 \\
13.2 \\
22.0\end{array}$ & $\begin{array}{r}7.9 \\
27.3 \\
43.9 \\
58.2\end{array}$ & $\begin{array}{l}13.7 \\
22.5 \\
23.1 \\
70.6\end{array}$ & $\begin{array}{r}3.5 \\
5.5 \\
5.9 \\
11.0\end{array}$ & $\begin{array}{l}16.5 \\
54.9 \\
31.9 \\
54.9\end{array}$ & $\begin{array}{l}2.2 \\
3.3 \\
4.4 \\
7.7\end{array}$ & $\begin{array}{r}2.4 \\
13.2 \\
17.6 \\
85.9\end{array}$ \\
\hline $\begin{array}{l}\text { Race or ethnicity of respondent } \\
\text { White non-Hispanic } \ldots \ldots \ldots \ldots \ldots \ldots \ldots \\
\text { Nonwhite or Hispanic } \ldots \ldots \ldots \ldots \ldots \ldots\end{array}$ & $\begin{array}{l}5.5 \\
1.6\end{array}$ & $\begin{array}{l}17.6 \\
13.2\end{array}$ & $\begin{array}{r}1.1 \\
.7\end{array}$ & $\begin{array}{c}87.9 \\
*\end{array}$ & $\begin{array}{r}19.8 \\
5.8\end{array}$ & $\begin{array}{l}49.4 \\
19.8\end{array}$ & $\begin{array}{l}45.0 \\
17.6\end{array}$ & $\begin{array}{l}7.7 \\
5.5\end{array}$ & $\begin{array}{l}49.4 \\
43.9\end{array}$ & $\begin{array}{l}5.5 \\
2.7\end{array}$ & $\begin{array}{r}39.5 \\
5.5\end{array}$ \\
\hline $\begin{array}{l}\text { Current work status of head } \\
\text { Working for someone else } \ldots \ldots \ldots \ldots \\
\text { Self-employed } \ldots \ldots \ldots \ldots \ldots \ldots \ldots \ldots \\
\text { Retired } \ldots \ldots \ldots \ldots \ldots \ldots \ldots \ldots \ldots \ldots \\
\text { Other not working } \ldots \ldots \ldots \ldots \ldots \ldots \ldots\end{array}$ & $\begin{array}{r}3.5 \\
11.0 \\
4.6 \\
2.2\end{array}$ & $\begin{array}{r}11.0 \\
22.0 \\
27.5 \\
8.8\end{array}$ & $\begin{array}{l}.8 \\
2.1 \\
3.3 \\
2.2\end{array}$ & $\begin{array}{r}27.5 \\
142.8 \\
98.8 \\
*\end{array}$ & $\begin{array}{r}11.0 \\
27.5 \\
49.4 \\
5.5\end{array}$ & $\begin{array}{l}27.5 \\
65.9 \\
82.4 \\
17.5\end{array}$ & $\begin{array}{l}32.9 \\
65.9 \\
51.6 \\
34.0\end{array}$ & $\begin{array}{r}5.9 \\
11.5 \\
5.5 \\
9.2\end{array}$ & $\begin{array}{l}54.9 \\
46.1 \\
49.4 \\
*\end{array}$ & $\begin{array}{r}3.3 \\
6.6 \\
11.0 \\
3.3\end{array}$ & $\begin{array}{r}22.5 \\
58.4 \\
29.1 \\
5.5\end{array}$ \\
\hline $\begin{array}{l}\text { Current occupation of head } \\
\text { Managerial or professional } \ldots \ldots \ldots \ldots \\
\text { Technical, sales, or services } \ldots \ldots \ldots \ldots \\
\text { Other occupation .................. } \\
\text { Retired or other not working } \ldots \ldots \ldots \ldots\end{array}$ & $\begin{array}{l}8.9 \\
2.6 \\
2.7 \\
3.9\end{array}$ & $\begin{array}{r}16.5 \\
13.2 \\
6.4 \\
24.2\end{array}$ & $\begin{array}{r}1.1 \\
.9 \\
.5 \\
2.3\end{array}$ & $\begin{array}{c}54.9 \\
38.4 \\
* \\
87.9\end{array}$ & $\begin{array}{r}22.0 \\
8.8 \\
5.5 \\
38.4\end{array}$ & $\begin{array}{l}44.4 \\
27.3 \\
22.0 \\
72.2\end{array}$ & $\begin{array}{l}65.9 \\
23.8 \\
22.0 \\
46.1\end{array}$ & $\begin{array}{r}11.0 \\
5.5 \\
5.5 \\
5.5\end{array}$ & $\begin{array}{l}49.4 \\
65.9 \\
39.5 \\
49.4\end{array}$ & $\begin{array}{l}6.6 \\
3.3 \\
2.2 \\
7.7\end{array}$ & $\begin{array}{l}73.2 \\
13.4 \\
12.5 \\
21.4\end{array}$ \\
\hline 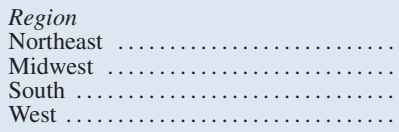 & $\begin{array}{l}6.6 \\
4.5 \\
3.3 \\
3.7\end{array}$ & $\begin{array}{l}19.8 \\
11.4 \\
15.4 \\
24.2\end{array}$ & $\begin{array}{r}1.6 \\
.9 \\
1.1 \\
.7\end{array}$ & $\begin{array}{r}164.7 \\
71.4 \\
43.9 \\
109.8\end{array}$ & $\begin{array}{l}16.5 \\
13.2 \\
17.6 \\
19.8\end{array}$ & $\begin{array}{l}54.9 \\
49.4 \\
49.4 \\
28.6\end{array}$ & $\begin{array}{l}57.9 \\
41.7 \\
29.7 \\
32.9\end{array}$ & $\begin{array}{l}6.6 \\
7.7 \\
5.5 \\
6.6\end{array}$ & $\begin{array}{l}54.9 \\
46.1 \\
49.4 \\
49.4\end{array}$ & $\begin{array}{l}4.4 \\
4.4 \\
4.1 \\
5.5\end{array}$ & $\begin{array}{l}47.4 \\
33.9 \\
13.4 \\
25.3\end{array}$ \\
\hline $\begin{array}{l}\text { Urbanicity } \\
\text { Metropolitan statistical area (MSA) } \ldots \\
\text { Non-MSA } \ldots \ldots \ldots \ldots \ldots \ldots \ldots \ldots \ldots\end{array}$ & $\begin{array}{l}4.6 \\
2.4\end{array}$ & $\begin{array}{l}16.5 \\
16.5\end{array}$ & $\begin{array}{l}1.1 \\
1.1\end{array}$ & $\begin{array}{c}87.9 \\
*\end{array}$ & $\begin{array}{r}18.7 \\
8.8\end{array}$ & $\begin{array}{l}54.9 \\
27.5\end{array}$ & $\begin{array}{l}43.9 \\
22.0\end{array}$ & $\begin{array}{l}6.9 \\
5.5\end{array}$ & $\begin{array}{l}49.4 \\
35.5\end{array}$ & $\begin{array}{l}5.3 \\
2.2\end{array}$ & $\begin{array}{l}30.3 \\
10.3\end{array}$ \\
\hline $\begin{array}{l}\text { Housing status } \\
\text { Owner } \ldots \ldots \ldots \ldots \ldots \ldots \ldots \ldots \\
\text { Renter or other } \ldots \ldots \ldots \ldots \ldots\end{array}$ & $\begin{array}{l}6.6 \\
1.2\end{array}$ & $\begin{array}{r}22.0 \\
7.7\end{array}$ & $\begin{array}{r}1.1 \\
.8\end{array}$ & $\begin{array}{r}71.4 \\
142.8\end{array}$ & $\begin{array}{r}22.0 \\
4.9\end{array}$ & $\begin{array}{l}54.9 \\
11.0\end{array}$ & $\begin{array}{l}50.5 \\
12.1\end{array}$ & $\begin{array}{l}7.7 \\
3.3\end{array}$ & $\begin{array}{l}49.4 \\
46.1\end{array}$ & $\begin{array}{l}6.6 \\
2.2\end{array}$ & $\begin{array}{r}52.6 \\
3.3\end{array}$ \\
\hline 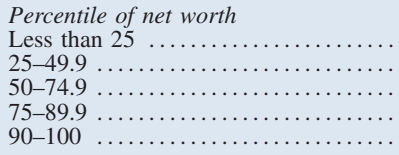 & $\begin{array}{r}.6 \\
2.2 \\
6.4 \\
17.4 \\
47.2\end{array}$ & $\begin{array}{r}2.2 \\
6.4 \\
11.4 \\
34.0 \\
50.5\end{array}$ & $\begin{array}{r}.3 \\
.5 \\
1.1 \\
2.2 \\
2.7\end{array}$ & $\begin{array}{c}* \\
* \\
* \\
27.5 \\
122.0\end{array}$ & $\begin{array}{r}2.1 \\
3.8 \\
8.8 \\
22.0 \\
120.8\end{array}$ & $\begin{array}{r}2.2 \\
8.1 \\
17.6 \\
54.9 \\
175.7\end{array}$ & $\begin{array}{r}3.2 \\
12.9 \\
36.8 \\
105.1 \\
289.9\end{array}$ & $\begin{array}{r}.9 \\
4.4 \\
5.5 \\
11.0 \\
22.0\end{array}$ & $\begin{array}{c}* \\
10.3 \\
24.1 \\
54.9 \\
148.3\end{array}$ & $\begin{array}{r}.8 \\
2.2 \\
5.5 \\
7.7 \\
43.9\end{array}$ & $\begin{array}{r}1.1 \\
10.9 \\
51.8 \\
223.0 \\
800.4\end{array}$ \\
\hline $\begin{array}{l}\text { MEмo } \\
\text { Mean value of holdings for } \\
\text { families holding asset } \ldots . .\end{array}$ & 29.8 & 60.2 & 6.3 & 600.8 & 176.1 & 202.0 & 133.2 & 25.3 & 227.4 & 43.4 & 220.4 \\
\hline
\end{tabular}

Note: See note to table 1.

* Ten or fewer observations. 
6. Family holdings of financial assets, by selected characteristics of families and type of asset, 2004 and 2007 surveysContinued

B. 2007 Survey of Consumer Finances

\begin{tabular}{|c|c|c|c|c|c|c|c|c|c|c|c|}
\hline Family characteristic & $\begin{array}{l}\text { Trans- } \\
\text { action } \\
\text { accounts }\end{array}$ & $\begin{array}{l}\text { Certifi- } \\
\text { cates of } \\
\text { deposit }\end{array}$ & $\begin{array}{c}\text { Savings } \\
\text { bonds }\end{array}$ & Bonds & Stocks & $\begin{array}{c}\text { Pooled } \\
\text { invest- } \\
\text { ment } \\
\text { funds }\end{array}$ & $\begin{array}{c}\text { Retire- } \\
\text { ment } \\
\text { accounts }\end{array}$ & $\begin{array}{c}\text { Cash } \\
\text { value life } \\
\text { insurance }\end{array}$ & $\begin{array}{c}\text { Other } \\
\text { managed } \\
\text { assets }\end{array}$ & Other & $\begin{array}{l}\text { Any } \\
\text { financial } \\
\text { asset }\end{array}$ \\
\hline & \multicolumn{11}{|c|}{ Percentage of families holding asset } \\
\hline All families $\ldots \ldots \ldots \ldots \ldots \ldots \ldots \ldots$ & 92.1 & 16.1 & 14.9 & 1.6 & 17.9 & 11.4 & 52.6 & 23.0 & 5.8 & 9.3 & 93.9 \\
\hline 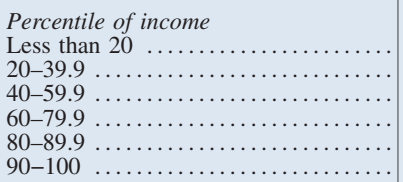 & $\begin{array}{r}74.9 \\
90.1 \\
96.4 \\
99.3 \\
100.0 \\
100.0\end{array}$ & $\begin{array}{r}9.4 \\
12.7 \\
15.4 \\
19.3 \\
19.9 \\
27.7\end{array}$ & $\begin{array}{r}3.6 \\
8.5 \\
15.2 \\
20.9 \\
26.2 \\
26.1\end{array}$ & $\begin{array}{l}* \\
* \\
* \\
1.4 \\
1.8 \\
8.9\end{array}$ & $\begin{array}{r}5.5 \\
7.8 \\
14.0 \\
23.2 \\
30.5 \\
47.5\end{array}$ & $\begin{array}{r}3.4 \\
4.6 \\
7.1 \\
14.6 \\
18.9 \\
35.5\end{array}$ & $\begin{array}{l}10.7 \\
35.6 \\
55.2 \\
73.3 \\
86.7 \\
89.6\end{array}$ & $\begin{array}{l}12.8 \\
16.4 \\
21.6 \\
29.4 \\
30.6 \\
38.9\end{array}$ & $\begin{array}{r}2.7 \\
4.7 \\
5.3 \\
5.7 \\
7.6 \\
13.6\end{array}$ & $\begin{array}{r}6.6 \\
8.8 \\
10.2 \\
8.4 \\
9.8 \\
15.3\end{array}$ & $\begin{array}{r}79.1 \\
93.2 \\
97.2 \\
99.7 \\
100.0 \\
100.0\end{array}$ \\
\hline 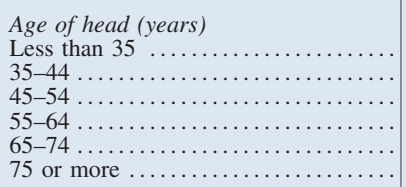 & $\begin{array}{l}87.3 \\
91.2 \\
91.7 \\
96.4 \\
94.6 \\
95.3\end{array}$ & $\begin{array}{r}6.7 \\
9.0 \\
14.3 \\
20.5 \\
24.2 \\
37.0\end{array}$ & $\begin{array}{r}13.7 \\
16.8 \\
19.0 \\
16.2 \\
10.3 \\
7.9\end{array}$ & $\begin{array}{l}* \\
.7 \\
1.1 \\
2.1 \\
4.2 \\
3.5\end{array}$ & $\begin{array}{l}13.7 \\
17.0 \\
18.6 \\
21.3 \\
19.1 \\
20.2\end{array}$ & $\begin{array}{r}5.3 \\
11.6 \\
12.6 \\
14.3 \\
14.6 \\
13.2\end{array}$ & $\begin{array}{l}41.6 \\
57.5 \\
64.7 \\
60.9 \\
51.7 \\
30.0\end{array}$ & $\begin{array}{l}11.4 \\
17.5 \\
22.3 \\
35.2 \\
34.4 \\
27.6\end{array}$ & $\begin{array}{r}* \\
2.2 \\
5.1 \\
7.7 \\
13.2 \\
14.0\end{array}$ & $\begin{array}{r}10.0 \\
9.6 \\
10.5 \\
9.2 \\
9.4 \\
5.3\end{array}$ & $\begin{array}{l}89.2 \\
93.1 \\
93.3 \\
97.8 \\
96.1 \\
97.4\end{array}$ \\
\hline $\begin{array}{l}\text { Family structure } \\
\text { Single with child(ren) } \ldots \ldots \ldots \ldots \ldots \\
\text { Single, no child, age less than } 55 \ldots \ldots \\
\text { Single, no child, age } 55 \text { or more } \ldots \ldots \\
\text { Couple with child(ren) } \ldots \ldots \ldots \ldots \ldots \ldots \\
\text { Couple, no child ........................ }\end{array}$ & $\begin{array}{l}84.8 \\
84.3 \\
94.3 \\
95.5 \\
94.8\end{array}$ & $\begin{array}{r}9.6 \\
9.6 \\
23.3 \\
15.1 \\
17.6\end{array}$ & $\begin{array}{r}10.1 \\
9.9 \\
9.9 \\
22.8 \\
17.1\end{array}$ & $\begin{array}{l}* \\
* \\
2.1 \\
1.2 \\
2.2\end{array}$ & $\begin{array}{r}8.4 \\
14.7 \\
13.1 \\
20.2 \\
21.5\end{array}$ & $\begin{array}{r}9.0 \\
7.7 \\
10.4 \\
13.6 \\
12.9\end{array}$ & $\begin{array}{l}36.1 \\
42.8 \\
36.2 \\
62.5 \\
61.8\end{array}$ & $\begin{array}{l}24.8 \\
11.4 \\
23.1 \\
27.5 \\
26.3\end{array}$ & $\begin{array}{r}* \\
1.6 \\
10.8 \\
5.3 \\
6.3\end{array}$ & $\begin{array}{r}13.2 \\
11.1 \\
7.6 \\
7.5 \\
9.0\end{array}$ & $\begin{array}{l}88.2 \\
86.9 \\
96.3 \\
96.2 \\
96.1\end{array}$ \\
\hline $\begin{array}{l}\text { Education of head } \\
\text { No high school diploma } \ldots \ldots \ldots \ldots \ldots \\
\text { High school diploma } \ldots \ldots \ldots \ldots \ldots \ldots \\
\text { Some college } \ldots \ldots \ldots \ldots \ldots \ldots \ldots \ldots \\
\text { College degree } \ldots \ldots \ldots \ldots \ldots \ldots \ldots \ldots\end{array}$ & $\begin{array}{l}75.7 \\
90.9 \\
93.9 \\
98.7\end{array}$ & $\begin{array}{r}9.5 \\
14.1 \\
14.1 \\
21.6\end{array}$ & $\begin{array}{r}3.4 \\
11.5 \\
16.4 \\
21.6\end{array}$ & $\begin{array}{l}* \\
.6 \\
1.2 \\
3.3\end{array}$ & $\begin{array}{r}3.9 \\
9.3 \\
17.4 \\
31.5\end{array}$ & $\begin{array}{r}2.2 \\
5.8 \\
8.9 \\
21.4\end{array}$ & $\begin{array}{l}21.6 \\
43.2 \\
52.5 \\
73.3\end{array}$ & $\begin{array}{l}12.6 \\
22.6 \\
23.4 \\
27.1\end{array}$ & $\begin{array}{l}1.7 \\
4.2 \\
6.6 \\
8.5\end{array}$ & $\begin{array}{r}7.1 \\
8.2 \\
9.8 \\
10.9\end{array}$ & $\begin{array}{l}79.7 \\
93.3 \\
95.5 \\
98.9\end{array}$ \\
\hline $\begin{array}{l}\text { Race or ethnicity of respondent } \\
\text { White non-Hispanic } \ldots \ldots \ldots \ldots \ldots \ldots \\
\text { Nonwhite or Hispanic } \ldots \ldots \ldots \ldots \ldots \ldots\end{array}$ & $\begin{array}{l}95.5 \\
83.9\end{array}$ & $\begin{array}{r}19.4 \\
8.2\end{array}$ & $\begin{array}{r}17.8 \\
7.8\end{array}$ & $\begin{array}{r}2.1 \\
.4\end{array}$ & $\begin{array}{r}21.4 \\
9.4\end{array}$ & $\begin{array}{r}13.7 \\
5.8\end{array}$ & $\begin{array}{l}58.2 \\
39.1\end{array}$ & $\begin{array}{l}25.3 \\
17.6\end{array}$ & $\begin{array}{l}7.3 \\
2.3\end{array}$ & $\begin{array}{l}9.7 \\
8.3\end{array}$ & $\begin{array}{l}96.8 \\
86.7\end{array}$ \\
\hline $\begin{array}{l}\text { Current work status of head } \\
\text { Working for someone else } \ldots \ldots \ldots \ldots \ldots \\
\text { Self-employed } \ldots \ldots \ldots \ldots \ldots \ldots \ldots \ldots \\
\text { Retired } \ldots \ldots \ldots \ldots \ldots \ldots \ldots \ldots \ldots \\
\text { Other not working } \ldots \ldots \ldots \ldots \ldots \ldots \ldots\end{array}$ & $\begin{array}{l}92.6 \\
96.9 \\
91.6 \\
78.6\end{array}$ & $\begin{array}{r}13.2 \\
15.0 \\
25.7 \\
5.6\end{array}$ & $\begin{array}{l}17.0 \\
15.9 \\
10.2 \\
10.7\end{array}$ & $\begin{array}{l}.9 \\
4.2 \\
2.3 \\
*\end{array}$ & $\begin{array}{l}17.8 \\
24.3 \\
16.4 \\
12.8\end{array}$ & $\begin{array}{r}10.4 \\
21.4 \\
11.3 \\
2.4\end{array}$ & $\begin{array}{l}62.1 \\
55.3 \\
34.2 \\
22.6\end{array}$ & $\begin{array}{l}20.3 \\
32.1 \\
27.3 \\
14.5\end{array}$ & $\begin{array}{r}3.7 \\
6.9 \\
11.2 \\
*\end{array}$ & $\begin{array}{r}9.2 \\
14.8 \\
7.0 \\
10.6\end{array}$ & $\begin{array}{l}94.1 \\
98.0 \\
93.7 \\
81.4\end{array}$ \\
\hline $\begin{array}{l}\text { Current occupation of head } \\
\text { Managerial or professional } \ldots \ldots \ldots \ldots \\
\text { Technical, sales, or services } \ldots \ldots \ldots \ldots \\
\text { Other occupation } \ldots \ldots \ldots \ldots \ldots \ldots \ldots \\
\text { Retired or other not working } \ldots \ldots \ldots \ldots\end{array}$ & $\begin{array}{l}98.3 \\
91.9 \\
87.9 \\
89.5\end{array}$ & $\begin{array}{r}18.2 \\
11.5 \\
9.2 \\
22.5\end{array}$ & $\begin{array}{l}21.1 \\
15.0 \\
13.1 \\
10.3\end{array}$ & $\begin{array}{l}3.1 \\
.4 \\
* \\
2.0\end{array}$ & $\begin{array}{r}28.7 \\
14.9 \\
9.9 \\
15.8\end{array}$ & $\begin{array}{r}19.7 \\
8.8 \\
5.4 \\
9.9\end{array}$ & $\begin{array}{l}74.1 \\
54.5 \\
51.0 \\
32.4\end{array}$ & $\begin{array}{l}24.9 \\
21.3 \\
19.0 \\
25.3\end{array}$ & $\begin{array}{l}6.7 \\
4.0 \\
1.1 \\
9.8\end{array}$ & $\begin{array}{r}11.1 \\
9.1 \\
9.6 \\
7.6\end{array}$ & $\begin{array}{l}98.7 \\
94.0 \\
90.2 \\
91.8\end{array}$ \\
\hline 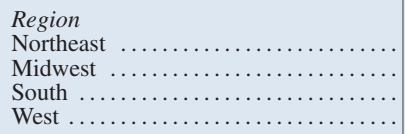 & $\begin{array}{l}91.3 \\
93.6 \\
91.3 \\
92.7\end{array}$ & $\begin{array}{l}18.1 \\
16.8 \\
15.1 \\
15.5\end{array}$ & $\begin{array}{l}18.9 \\
16.0 \\
12.0 \\
15.0\end{array}$ & $\begin{array}{l}2.0 \\
1.2 \\
1.7 \\
1.6\end{array}$ & $\begin{array}{l}21.4 \\
17.9 \\
15.4 \\
19.2\end{array}$ & $\begin{array}{r}15.5 \\
10.6 \\
9.7 \\
11.5\end{array}$ & $\begin{array}{l}53.3 \\
57.8 \\
48.8 \\
52.9\end{array}$ & $\begin{array}{l}23.5 \\
26.6 \\
23.3 \\
18.3\end{array}$ & $\begin{array}{l}6.4 \\
6.7 \\
5.2 \\
5.5\end{array}$ & $\begin{array}{r}5.4 \\
9.2 \\
8.6 \\
13.9\end{array}$ & $\begin{array}{l}92.5 \\
95.4 \\
93.5 \\
93.9\end{array}$ \\
\hline $\begin{array}{l}\text { Urbanicity } \\
\text { Metropolitan statistical area (MSA) } \ldots \\
\text { Non-MSA } \ldots \ldots \ldots \ldots \ldots \ldots \ldots \ldots \ldots\end{array}$ & $\begin{array}{l}92.8 \\
88.7\end{array}$ & $\begin{array}{l}16.2 \\
15.9\end{array}$ & $\begin{array}{l}15.1 \\
13.8\end{array}$ & $\begin{array}{r}1.8 \\
.8\end{array}$ & $\begin{array}{l}19.4 \\
10.9\end{array}$ & $\begin{array}{r}12.1 \\
7.7\end{array}$ & $\begin{array}{l}54.8 \\
42.0\end{array}$ & $\begin{array}{l}22.2 \\
26.7\end{array}$ & $\begin{array}{l}5.9 \\
5.5\end{array}$ & $\begin{array}{l}9.5 \\
8.6\end{array}$ & $\begin{array}{l}94.3 \\
91.8\end{array}$ \\
\hline $\begin{array}{l}\text { Housing status } \\
\text { Owner } \ldots \ldots \ldots \ldots \ldots \ldots \ldots \ldots \ldots \ldots \ldots \ldots \ldots \ldots \ldots \\
\text { Renter or other } \ldots \ldots \ldots \ldots \ldots \ldots \ldots\end{array}$ & $\begin{array}{l}97.3 \\
80.8\end{array}$ & $\begin{array}{r}20.0 \\
7.7\end{array}$ & $\begin{array}{r}18.2 \\
7.5\end{array}$ & $\begin{array}{r}2.2 \\
.4\end{array}$ & $\begin{array}{r}22.4 \\
8.1\end{array}$ & $\begin{array}{r}15.0 \\
3.5\end{array}$ & $\begin{array}{l}63.3 \\
29.2\end{array}$ & $\begin{array}{l}28.9 \\
10.1\end{array}$ & $\begin{array}{l}7.5 \\
2.1\end{array}$ & $\begin{array}{l}9.4 \\
9.1\end{array}$ & $\begin{array}{l}98.4 \\
84.0\end{array}$ \\
\hline 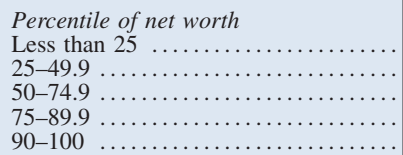 & $\begin{array}{r}76.4 \\
93.6 \\
98.6 \\
100.0 \\
100.0\end{array}$ & $\begin{array}{r}2.5 \\
9.9 \\
19.3 \\
32.6 \\
33.0\end{array}$ & $\begin{array}{r}4.7 \\
12.3 \\
17.5 \\
25.9 \\
23.3\end{array}$ & $\begin{array}{c}* \\
* \\
* \\
* \\
11.8\end{array}$ & $\begin{array}{r}4.3 \\
10.2 \\
17.3 \\
31.6 \\
52.3\end{array}$ & $\begin{array}{r}* \\
3.6 \\
10.5 \\
22.5 \\
42.5\end{array}$ & $\begin{array}{l}19.1 \\
48.1 \\
62.9 \\
77.4 \\
84.6\end{array}$ & $\begin{array}{r}7.8 \\
19.7 \\
28.5 \\
32.1 \\
41.9\end{array}$ & $\begin{array}{c}* \\
1.9 \\
6.2 \\
11.2 \\
20.3\end{array}$ & $\begin{array}{r}7.4 \\
8.8 \\
8.8 \\
9.4 \\
16.6\end{array}$ & $\begin{array}{r}79.6 \\
96.4 \\
99.5 \\
100.0 \\
100.0\end{array}$ \\
\hline
\end{tabular}


6. Family holdings of financial assets, by selected characteristics of families and type of asset, 2004 and 2007 surveysContinued

B. 2007 Survey of Consumer Finances—continued

\begin{tabular}{|c|c|c|c|c|c|c|c|c|c|c|c|}
\hline Family characteristic & $\begin{array}{l}\text { Trans- } \\
\text { action } \\
\text { accounts }\end{array}$ & $\begin{array}{l}\text { Certifi- } \\
\text { cates of } \\
\text { deposit }\end{array}$ & $\begin{array}{l}\text { Savings } \\
\text { bonds }\end{array}$ & Bonds & Stocks & $\begin{array}{l}\text { Pooled } \\
\text { invest- } \\
\text { ment } \\
\text { funds }\end{array}$ & $\begin{array}{c}\text { Retire- } \\
\text { ment } \\
\text { accounts }\end{array}$ & $\begin{array}{c}\text { Cash } \\
\text { value life } \\
\text { insurance }\end{array}$ & $\begin{array}{c}\text { Other } \\
\text { managed } \\
\text { assets }\end{array}$ & Other & $\begin{array}{l}\text { Any } \\
\text { financial } \\
\text { asset }\end{array}$ \\
\hline & \multicolumn{11}{|c|}{ Median value of holdings for families holding asset (thousands of 2007 dollars) } \\
\hline All families & 4.0 & 20.0 & 1.0 & 80.0 & 17.0 & 56.0 & 45.0 & 8.0 & 70.0 & 6.0 & 28.8 \\
\hline 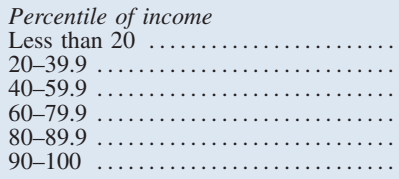 & $\begin{array}{r}.8 \\
1.6 \\
2.7 \\
6.0 \\
12.9 \\
36.7\end{array}$ & $\begin{array}{l}18.0 \\
18.0 \\
17.0 \\
11.0 \\
20.0 \\
42.0\end{array}$ & $\begin{array}{r}.5 \\
1.0 \\
.7 \\
1.0 \\
2.0 \\
2.5\end{array}$ & $\begin{array}{r}* \\
* \\
* \\
19.0 \\
81.0 \\
250.0\end{array}$ & $\begin{array}{r}3.8 \\
10.0 \\
5.5 \\
14.0 \\
15.0 \\
75.0\end{array}$ & $\begin{array}{r}30.0 \\
30.0 \\
37.5 \\
35.0 \\
46.0 \\
180.0\end{array}$ & $\begin{array}{r}6.5 \\
12.0 \\
23.9 \\
48.0 \\
85.0 \\
200.0\end{array}$ & $\begin{array}{r}2.5 \\
5.0 \\
5.2 \\
10.0 \\
9.0 \\
28.1\end{array}$ & $\begin{array}{r}100.0 \\
86.0 \\
59.0 \\
52.0 \\
30.0 \\
90.0\end{array}$ & $\begin{array}{r}1.5 \\
3.0 \\
4.0 \\
10.0 \\
10.0 \\
45.0\end{array}$ & $\begin{array}{r}1.7 \\
7.0 \\
18.6 \\
58.3 \\
129.9 \\
404.5\end{array}$ \\
\hline 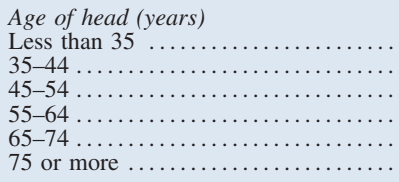 & $\begin{array}{l}2.4 \\
3.4 \\
5.0 \\
5.2 \\
7.7 \\
6.1\end{array}$ & $\begin{array}{r}5.0 \\
5.0 \\
15.0 \\
23.0 \\
23.2 \\
30.0\end{array}$ & $\begin{array}{r}.7 \\
1.0 \\
1.0 \\
1.9 \\
1.0 \\
20.0\end{array}$ & $\begin{array}{r}* \\
9.7 \\
200.0 \\
90.8 \\
50.0 \\
100.0\end{array}$ & $\begin{array}{r}3.0 \\
15.0 \\
18.5 \\
24.0 \\
38.0 \\
40.0\end{array}$ & $\begin{array}{r}18.0 \\
22.5 \\
50.0 \\
112.0 \\
86.0 \\
75.0\end{array}$ & $\begin{array}{l}10.0 \\
36.0 \\
67.0 \\
98.0 \\
77.0 \\
35.0\end{array}$ & $\begin{array}{r}2.8 \\
8.3 \\
10.0 \\
10.0 \\
10.0 \\
5.0\end{array}$ & $\begin{array}{r}* \\
24.0 \\
45.0 \\
59.0 \\
70.0 \\
100.0\end{array}$ & $\begin{array}{r}1.5 \\
8.0 \\
6.0 \\
20.0 \\
10.0 \\
15.0\end{array}$ & $\begin{array}{r}6.8 \\
25.8 \\
54.0 \\
72.4 \\
68.1 \\
41.5\end{array}$ \\
\hline $\begin{array}{l}\text { Family structure } \\
\text { Single with child(ren) } \ldots \ldots \ldots \ldots \ldots \\
\text { Single, no child, age less than } 55 \ldots \ldots \\
\text { Single, no child, age } 55 \text { or more } \ldots \ldots \\
\text { Couple with child(ren) } \ldots \ldots \ldots \ldots \ldots \ldots \\
\text { Couple, no child ....................... }\end{array}$ & $\begin{array}{l}2.4 \\
2.0 \\
2.5 \\
5.0 \\
6.0\end{array}$ & $\begin{array}{r}7.5 \\
5.5 \\
28.0 \\
10.0 \\
20.0\end{array}$ & $\begin{array}{r}1.0 \\
1.5 \\
3.0 \\
.8 \\
1.0\end{array}$ & $\begin{array}{r}* \\
* \\
50.0 \\
530.0 \\
80.0\end{array}$ & $\begin{array}{r}13.0 \\
3.8 \\
25.0 \\
15.0 \\
24.0\end{array}$ & $\begin{array}{l}46.0 \\
18.0 \\
77.0 \\
45.0 \\
60.0\end{array}$ & $\begin{array}{l}30.0 \\
20.0 \\
45.0 \\
52.0 \\
55.1\end{array}$ & $\begin{array}{r}5.0 \\
5.2 \\
5.0 \\
9.0 \\
10.0\end{array}$ & $\begin{array}{r}* \\
50.0 \\
100.0 \\
30.0 \\
52.0\end{array}$ & $\begin{array}{r}5.5 \\
3.0 \\
3.6 \\
10.0 \\
10.0\end{array}$ & $\begin{array}{r}10.3 \\
8.9 \\
24.4 \\
36.3 \\
46.1\end{array}$ \\
\hline $\begin{array}{l}\text { Education of head } \\
\text { No high school diploma } \ldots \ldots \ldots \ldots \ldots \\
\text { High school diploma } \ldots \ldots \ldots \ldots \ldots \ldots \\
\text { Some college } \ldots \ldots \ldots \ldots \ldots \ldots \ldots \ldots \\
\text { College degree } \ldots \ldots \ldots \ldots \ldots \ldots \ldots\end{array}$ & $\begin{array}{r}1.2 \\
2.5 \\
2.8 \\
10.0\end{array}$ & $\begin{array}{l}14.0 \\
16.0 \\
18.0 \\
25.0\end{array}$ & $\begin{array}{l}1.0 \\
1.0 \\
1.0 \\
1.1\end{array}$ & $\begin{array}{r}* \\
46.5 \\
50.0 \\
100.0\end{array}$ & $\begin{array}{r}2.7 \\
10.0 \\
6.0 \\
25.0\end{array}$ & $\begin{array}{l}64.0 \\
30.0 \\
25.0 \\
75.0\end{array}$ & $\begin{array}{l}15.0 \\
28.5 \\
32.0 \\
75.0\end{array}$ & $\begin{array}{r}2.5 \\
5.2 \\
8.0 \\
13.0\end{array}$ & $\begin{array}{l}30.0 \\
80.0 \\
52.0 \\
75.0\end{array}$ & $\begin{array}{r}1.5 \\
5.0 \\
4.0 \\
10.0\end{array}$ & $\begin{array}{r}3.0 \\
14.2 \\
20.0 \\
95.7\end{array}$ \\
\hline $\begin{array}{l}\text { Race or ethnicity of respondent } \\
\text { White non-Hispanic } \ldots \ldots \ldots \ldots \ldots \ldots \ldots \\
\text { Nonwhite or Hispanic } \ldots \ldots \ldots \ldots \ldots \ldots\end{array}$ & $\begin{array}{l}5.1 \\
2.0\end{array}$ & $\begin{array}{l}20.0 \\
10.0\end{array}$ & $\begin{array}{l}1.0 \\
1.0\end{array}$ & $\begin{array}{l}95.9 \\
23.1\end{array}$ & $\begin{array}{r}19.0 \\
8.0\end{array}$ & $\begin{array}{l}64.0 \\
30.0\end{array}$ & $\begin{array}{l}52.7 \\
25.4\end{array}$ & $\begin{array}{l}9.0 \\
5.0\end{array}$ & $\begin{array}{l}70.0 \\
30.0\end{array}$ & $\begin{array}{r}10.0 \\
3.0\end{array}$ & $\begin{array}{r}44.3 \\
9.0\end{array}$ \\
\hline $\begin{array}{l}\text { Current work status of head } \\
\text { Working for someone else } \ldots \ldots \ldots \ldots \\
\text { Self-employed } \ldots \ldots \ldots \ldots \ldots \ldots \ldots \ldots \\
\text { Retired } \ldots \ldots \ldots \ldots \ldots \ldots \ldots \ldots \ldots \ldots \\
\text { Other not working } \ldots \ldots \ldots \ldots \ldots \ldots \ldots\end{array}$ & $\begin{array}{l}3.8 \\
9.9 \\
4.0 \\
1.0\end{array}$ & $\begin{array}{l}10.0 \\
25.0 \\
30.0 \\
15.0\end{array}$ & $\begin{array}{l}1.0 \\
1.0 \\
2.5 \\
2.0\end{array}$ & $\begin{array}{r}46.8 \\
150.0 \\
79.5 \\
*\end{array}$ & $\begin{array}{r}10.5 \\
60.0 \\
28.7 \\
6.3\end{array}$ & $\begin{array}{l}42.0 \\
80.0 \\
78.2 \\
50.0\end{array}$ & $\begin{array}{l}40.0 \\
91.0 \\
48.0 \\
20.8\end{array}$ & $\begin{array}{r}7.5 \\
24.0 \\
5.5 \\
2.2\end{array}$ & $\begin{array}{r}27.2 \\
80.0 \\
100.0 \\
*\end{array}$ & $\begin{array}{r}5.0 \\
16.0 \\
10.0 \\
3.0\end{array}$ & $\begin{array}{r}28.5 \\
54.1 \\
29.7 \\
3.7\end{array}$ \\
\hline $\begin{array}{l}\text { Current occupation of head } \\
\text { Managerial or professional } \ldots \ldots \ldots \ldots \\
\text { Technical, sales, or services } \ldots \ldots \ldots \ldots \\
\text { Other occupation .................. } \\
\text { Retired or other not working } \ldots \ldots \ldots \ldots\end{array}$ & $\begin{array}{l}8.8 \\
3.0 \\
2.5 \\
3.3\end{array}$ & $\begin{array}{l}15.0 \\
15.0 \\
10.0 \\
30.0\end{array}$ & $\begin{array}{r}1.0 \\
1.0 \\
.7 \\
2.0\end{array}$ & $\begin{array}{r}80.0 \\
123.2 \\
* \\
95.9\end{array}$ & $\begin{array}{r}20.0 \\
12.0 \\
4.0 \\
25.0\end{array}$ & $\begin{array}{l}75.0 \\
40.0 \\
18.0 \\
78.2\end{array}$ & $\begin{array}{l}72.0 \\
30.0 \\
24.3 \\
45.0\end{array}$ & $\begin{array}{r}13.0 \\
9.0 \\
5.0 \\
5.0\end{array}$ & $\begin{array}{r}59.0 \\
10.0 \\
20.0 \\
100.0\end{array}$ & $\begin{array}{r}10.0 \\
5.0 \\
5.0 \\
5.5\end{array}$ & $\begin{array}{l}77.0 \\
17.6 \\
13.8 \\
23.7\end{array}$ \\
\hline 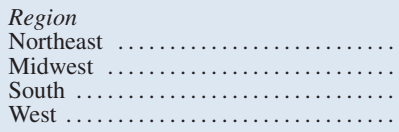 & $\begin{array}{l}5.1 \\
3.8 \\
3.5 \\
4.3\end{array}$ & $\begin{array}{l}20.0 \\
12.0 \\
20.0 \\
23.0\end{array}$ & $\begin{array}{l}1.0 \\
1.0 \\
1.2 \\
1.0\end{array}$ & $\begin{array}{r}114.7 \\
49.3 \\
100.0 \\
60.0\end{array}$ & $\begin{array}{l}17.9 \\
14.0 \\
17.9 \\
18.0\end{array}$ & $\begin{array}{l}50.0 \\
37.5 \\
70.0 \\
58.8\end{array}$ & $\begin{array}{l}57.5 \\
36.0 \\
40.0 \\
45.6\end{array}$ & $\begin{array}{r}9.0 \\
7.0 \\
8.0 \\
10.0\end{array}$ & $\begin{array}{l}73.0 \\
67.0 \\
80.0 \\
60.0\end{array}$ & $\begin{array}{r}10.0 \\
6.0 \\
4.0 \\
6.0\end{array}$ & $\begin{array}{l}43.8 \\
31.0 \\
20.8 \\
29.1\end{array}$ \\
\hline $\begin{array}{l}\text { Urbanicity } \\
\text { Metropolitan statistical area (MSA) } \ldots \\
\text { Non-MSA ....................... }\end{array}$ & $\begin{array}{l}4.5 \\
2.5\end{array}$ & $\begin{array}{l}20.0 \\
10.0\end{array}$ & $\begin{array}{l}1.0 \\
1.2\end{array}$ & $\begin{array}{r}100.0 \\
50.0\end{array}$ & $\begin{array}{l}19.0 \\
11.0\end{array}$ & $\begin{array}{l}60.0 \\
34.0\end{array}$ & $\begin{array}{l}48.0 \\
31.3\end{array}$ & $\begin{array}{l}9.0 \\
5.0\end{array}$ & $\begin{array}{l}70.0 \\
45.0\end{array}$ & $\begin{array}{l}8.0 \\
2.4\end{array}$ & $\begin{array}{l}32.6 \\
15.8\end{array}$ \\
\hline $\begin{array}{l}\text { Housing status } \\
\text { Owner } \ldots \ldots \ldots \ldots \ldots \ldots \ldots \ldots \\
\text { Renter or other } \ldots \ldots \ldots \ldots \ldots\end{array}$ & $\begin{array}{l}6.2 \\
1.2\end{array}$ & $\begin{array}{l}20.0 \\
10.0\end{array}$ & $\begin{array}{r}1.0 \\
.7\end{array}$ & $\begin{array}{r}100.0 \\
15.0\end{array}$ & $\begin{array}{r}20.0 \\
5.5\end{array}$ & $\begin{array}{l}60.0 \\
40.0\end{array}$ & $\begin{array}{l}57.0 \\
10.0\end{array}$ & $\begin{array}{r}10.0 \\
2.0\end{array}$ & $\begin{array}{l}70.0 \\
54.0\end{array}$ & $\begin{array}{r}10.0 \\
1.8\end{array}$ & $\begin{array}{r}54.3 \\
3.8\end{array}$ \\
\hline 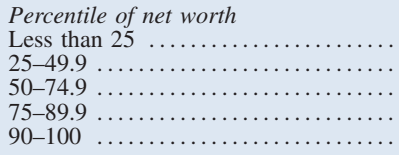 & $\begin{array}{r}.7 \\
2.0 \\
6.1 \\
15.5 \\
46.5\end{array}$ & $\begin{array}{r}2.0 \\
7.0 \\
15.0 \\
25.0 \\
50.0\end{array}$ & $\begin{array}{r}.5 \\
.7 \\
1.2 \\
2.0 \\
3.5\end{array}$ & $\begin{array}{c}* \\
* \\
* \\
* \\
150.0\end{array}$ & $\begin{array}{r}1.1 \\
3.0 \\
6.0 \\
20.0 \\
125.0\end{array}$ & $\begin{array}{r}* \\
9.0 \\
25.0 \\
50.0 \\
264.0\end{array}$ & $\begin{array}{r}3.2 \\
15.0 \\
48.6 \\
117.0 \\
314.0\end{array}$ & $\begin{array}{r}1.2 \\
3.0 \\
6.5 \\
15.0 \\
30.0\end{array}$ & $\begin{array}{c}* \\
13.8 \\
50.0 \\
80.0 \\
180.0\end{array}$ & $\begin{array}{r}1.2 \\
3.0 \\
10.0 \\
20.0 \\
50.0\end{array}$ & $\begin{array}{r}1.4 \\
13.2 \\
59.6 \\
215.0 \\
773.0\end{array}$ \\
\hline $\begin{array}{l}\text { MEмo } \\
\text { Mean value of holdings for } \\
\text { families holding asset } \ldots . .\end{array}$ & 26.4 & 55.6 & 6.6 & 574.3 & 221.1 & 309.7 & 145.8 & 31.3 & 248.8 & 50.3 & 235.8 \\
\hline
\end{tabular}

Note: See note to table 1.

* Ten or fewer observations. 
period, at 93.9 percent (first half of tables 6.A and 6.B, last column). However, the recent data show changes for some demographic groups. By income percentile groups, ownership fell for the first and third quintiles and rose or stayed the same for other income groups; by age, an increase appeared only for the 55-to-64 age group; by family structure, ownership increased for childless couples and childless single families headed by a person older than age 55 but declined for other single families; and by work status, ownership rose substantially for families headed by a person who was self-employed or neither working nor retired. Ownership increased for nonwhite or Hispanic families and decreased for white nonHispanic families. The share of homeowners with financial assets rose, but the ownership rate fell for renters.

In contrast to the drop in the overall ratio of financial assets to total assets over the recent period, the median holding of financial assets for families having such assets rose 13.8 percent (second half of tables 6.A and 6.B, last column), while the mean rose 7.0 percent (memo line). The recent change in the median did not completely offset the decrease over the previous three-year period. The more detailed picture is one of increases in the medians over the recent period for most demographic groups, including substantial increases for the lowest two income quintiles and all age groups except the 55-to-64 and 75-or-more categories. Median holdings increased most markedly for single families with children and younger childless single families; for families in the 65-to-75 age group; for families living in the South or outside of MSAs; and for nonwhite or Hispanic families. Mean holdings of those with financial assets generally rose; among the scattered declines, the largest was a 52.0 percent drop for families in the other-not-working work-status group (means by groups are not shown in the tables).

\section{Transaction Accounts and Certificates of Deposit}

In 2007, 92.1 percent of families had some type of transaction account-a category comprising checking, savings, and money market deposit accounts; money market mutual funds; and call or cash accounts at brokerages. The increase of 0.8 percentage point in ownership since 2004 resumed the upward trend seen in earlier surveys after the ownership rate had remained essentially unchanged over the previous three-year period. Families that did not have any type of transaction account in 2007 were disproportionately likely to be in the bottom income quintile group, to be headed by a person younger than 35 , to be nonwhite or Hispanic, to be headed by a person who was neither working nor retired, to be renters, or to have net worth in the bottom quartile. See box "Decisions about Checking Accounts" for a discussion of the reasons families do or do not have a checking account. Over the 2004-07 period, transaction account ownership rose noticeably-by 3 to 4 percentage points-for families in South, nonwhite or Hispanic families, and families headed by a person who did not graduate from high school or who was aged 55 to 64 .

The slight overall expansion in ownership of transaction accounts in the recent three-year period is reflected in the small changes in the types of transaction accounts held by families. Ownership of checking and savings accounts inched up, while ownership of money market and call accounts slightly declined (table 6.1).

6.1.

\begin{tabular}{c|c|c}
\hline \multirow{2}{*}{ Type of transaction account } & \multicolumn{2}{|c}{ All families } \\
\cline { 2 - 3 } & $\begin{array}{c}2007 \\
\text { (percent) }\end{array}$ & $\begin{array}{c}\text { Change, 2004-07 } \\
\text { (percentage points) }\end{array}$ \\
\hline Checking $\ldots \ldots \ldots \ldots \ldots \ldots \ldots$ & 89.7 & .3 \\
Savings $\ldots \ldots \ldots \ldots \ldots \ldots \ldots \ldots$ & 47.2 & .1 \\
Money market $\ldots \ldots \ldots \ldots \ldots \ldots \ldots$ & 20.9 & -.2 \\
Call $\ldots \ldots \ldots \ldots \ldots \ldots \ldots \ldots \ldots$ & 2.1 & -.4 \\
\hline
\end{tabular}

The savings account category includes a relatively small number of tax-preferred accounts such as medical or health savings accounts and Coverdell or 529 education accounts. ${ }^{21}$ For families with a savings account, ownership of any of these types of taxpreferred accounts increased, from 2.5 percent in 2004 to 3.8 percent in 2007. In both of these survey years, 529 plans accounted for about 80 percent of the number of tax-preferred savings accounts.

Median holdings in transaction accounts for those who had such accounts fell 2.4 percent from 2004 to 2007, while the mean fell 11.4 percent. Across demographic groups, the patterns of changes in the median are mainly a mixture of substantial increases and decreases. Median balances rose for the lowest and highest income groups and the lowest net worth quartile and fell or was unchanged for the middle income groups and all the other wealth groups; across age groups, the median increased substantially for the less-than-35 and the 65-to-74 age groups and fell or rose slightly for other families. By family structure, median balances increased sharply for single families with children and rose for childless single families headed by a person aged less than 55, but they fell for other families. Across work-status groups, median

21. Coverdell savings accounts, formerly known as education individual retirement accounts, and 529 saving plans are tax-deferred plans that parents or others may use to save for educational expenses. 


\section{Decisions about Checking Accounts}

Between 2004 and 2007, the proportion of families with any type of transaction account edged up slightly (table 6 in the main text), while the share without a checking account fell 0.3 percentage point, from 10.6 percent to 10.3 percent (data not shown in the tables). The decline in the fraction of families without a checking account follows a longer trend; in 1989, the share was 18.7 percent. $^{1}$

Among families without a checking account in 2007, 52.7 percent had held such an account in the past, 63.2 percent had incomes in the lowest quintile of that distribution, 56.3 percent were headed by a person younger than 45 , and 58.3 percent were nonwhite or Hispanic. The SCF asked all families that did not have a checking account to give a reason for not having an account (table A). The most commonly reported reason-

A. Distribution of reasons cited by respondents for their families' not having a checking account, by reason, 1998-2007 surveys

Percent

\begin{tabular}{|c|c|c|c|c|}
\hline Reason & 1998 & 2001 & 2004 & 2007 \\
\hline $\begin{array}{l}\text { Do not write enough checks to } \\
\text { make it worthwhile }\end{array}$ & 28.4 & 28.5 & 27.9 & 18.7 \\
\hline Minimum balance is too high. & 8.6 & 6.5 & 5.6 & 7.6 \\
\hline Do not like dealing with banks & 18.5 & 22.6 & 22.6 & 25.2 \\
\hline Service charges are too high ... & 11.0 & 10.2 & 11.6 & 12.3 \\
\hline $\begin{array}{l}\text { Cannot manage or balance a } \\
\text { checking account } \ldots . . . . . .\end{array}$ & 7.2 & 6.6 & 6.8 & 3.9 \\
\hline $\begin{array}{l}\text { No bank has convenient hours } \\
\text { or location }\end{array}$ & 1.2 & 4 & 1.1 & .8 \\
\hline Do not have enough money ... & 12.9 & 14.0 & 14.4 & 10.4 \\
\hline Credit problems....$\ldots \ldots \ldots$ & 2.7 & 3.6 & 2.4 & 6.6 \\
\hline Do not need or want account & 6.3 & 5.1 & 5.2 & 8.9 \\
\hline Other $\ldots \ldots \ldots \ldots$ & 3.1 & 2.3 & 2.4 & 5.6 \\
\hline Total & 100 & 100 & 100 & 100 \\
\hline
\end{tabular}

given by 25.2 percent of such families - was that the family did not like dealing with banks. Another 18.7 percent did not write enough checks to make account ownership worthwhile; this reason had been the most frequently reported one in each of the earlier years shown. The proportion reporting they did not have enough money to make an account worthwhile also declined notably-from 14.4 percent in 2004 to 10.4 percent in 2007. Another 12.3 percent of families said that service charges were too high. The SCF showed a sizable increase in the fraction of families reporting credit problems as a reason-from 2.4 percent in 2004 to 6.6 percent in 2007; the fraction of families that cited they did not need or want an account as a reason also increased substantially, from 5.2 percent in 2004 to 8.9 percent in 2007.

When attention is further restricted to families that once had a checking account (data not shown in the

1. For the definition of "transaction account," see the main text. For a more extensive discussion of the ways that families obtain checking and credit services, see Jeanne M. Hogarth, Christoslav E. Anguelov, and Jinkook Lee (2005), "Who Has a Bank Account? Exploring Changes over Time, 1989-2001," Journal of Family \& Economic Issues, vol. 26 (1), pp. 7-30. tables), the general pattern of responses is similar to that for all families without a checking account, but some differences are evident. For families that once had a checking account, the proportion reporting that they did not like banks, found service charges too high, or had credit problems all rose from 2004. These increases were offset by decreases in the proportion reporting that they did not write enough checks, could not manage or balance a checking account, or did not have enough money for an account to be worthwhile.

The SCF asked all families with a checking account to give the most important reason they chose the financial institution for their main checking account (table B). In

B. Distribution of reasons cited by respondents as the most important reason for choosing institution for their main checking account, 1998-2007 surveys Percent

\begin{tabular}{|c|c|c|c|c|}
\hline Reason & 1998 & 2001 & 2004 & 2007 \\
\hline Location of their offices ...... & 43.6 & 42.8 & 45.4 & 45.9 \\
\hline Had the lowest fees/minimum & & & & \\
\hline balance requirement $\ldots . \ldots$. & 18.4 & 16.6 & 16.3 & 13.7 \\
\hline $\begin{array}{l}\text { Able to obtain many services } \\
\text { at one place } \ldots \ldots \ldots \ldots \ldots \ldots\end{array}$ & 16.0 & 16.4 & 15.3 & 16.2 \\
\hline Recommended; friend/family & & & & \\
\hline has account there $\ldots \ldots \ldots$. & 3.6 & 4.7 & 3.9 & 4.2 \\
\hline $\begin{array}{l}\text { Personal relationship; they } \\
\text { know me; family member } \\
\text { works there } . . . \ldots \ldots \ldots \ldots\end{array}$ & 3.9 & 4.0 & 3.5 & 4.2 \\
\hline $\begin{array}{l}\text { Connection through work } \\
\text { or school } \ldots \ldots \ldots \ldots \ldots . .\end{array}$ & 1.4 & 2.0 & 3.5 & 3.3 \\
\hline $\begin{array}{l}\text { Always done business there; } \\
\text { banked there a long time; } \\
\text { other business there ...... }\end{array}$ & 2.7 & 2.4 & 2.9 & 3.0 \\
\hline $\begin{array}{l}\text { Offered safety and absence } \\
\text { of risk } \ldots \ldots \ldots \ldots \ldots \ldots\end{array}$ & 2.1 & 2.2 & 1.9 & 2.9 \\
\hline $\begin{array}{l}\text { Other convenience; payroll } \\
\text { deduction/direct deposit }\end{array}$ & 1.2 & 1.3 & 1.2 & 1.0 \\
\hline Other $\ldots \ldots \ldots \ldots \ldots \ldots$ & 7.1 & 7.5 & 6.1 & 6.1 \\
\hline Total & 100 & 100 & 100 & 100 \\
\hline
\end{tabular}

2007, 45.9 percent of families chose the institution for their main checking account for reasons related to the location of the offices of the institution. ${ }^{2}$ Another 16.2 percent placed the most importance on the ability to obtain many services at one place, and 13.7 percent singled out the importance of obtaining the lowest fees or minimum balance requirements. Absence of risk was of primary importance for only a relatively small fraction of families. Over the 2004-07 period, the most noticeable changes in these responses were a decrease in the fraction of families citing reasons related to the lowest fees or minimum balance requirements and the increase in the fraction citing reasons related to the safety and absence of risk offered by the institution.

2. For a discussion of the definition of local banking markets, see Dean F. Amel, Arthur B. Kennickell, and Kevin B. Moore (2008), "Banking Market Definition: Evidence from the Survey of Consumer Finances," Finance and Economics Discussion Series 2008-35 (Washington: Board of Governors of the Federal Reserve System, October), www.federalreserve.gov/pubs/feds/2008/200835/200835pap.pdf. 
balances fell for all groups except the working-forsomeone-else category. Holdings increased for households headed by a person in a technical, sales, or service occupation but decreased for the remaining three occupation groups. Median balances increased strongly for nonwhite or Hispanic families and fell somewhat for other families. By region, median holdings declined substantially for families in the Northeast and Midwest.

Certificates of deposit (CDs) - interest-bearing deposits with a set term-are traditionally viewed as a low-risk saving vehicle, and they are often used by persons who desire a safe haven from the volatility of financial markets. Over the 2004-07 period, the attractiveness of CDs increased as the interest rates on them rose. The resulting increase of 3.4 percentage points in ownership was the largest increase observed in the SCF since 1989. Over the recent period, ownership increased among almost all demographic groups. Increases in ownership were particularly strong for the top income group, the oldest age group, retired families, and the next-to-highest net worth group. The overall median value of holdings of CDs increased 21.2 percent over the three-year period, while the mean value decreased 7.6 percent. Consideration of changes in the median across demographic groups reveals substantial increases for the first and third income quintiles, the some-college education group, the other-not-working group, and the other occupation group. The overall decline in the mean suggests that balances on most new accounts tended to be moderate.

\section{Savings Bonds and Other Bonds}

Savings bonds are owned disproportionately by families in the highest 40 percent of the income distribution and by families in the top half of the distribution of net worth. Over the 2004-07 period, the ownership of savings bonds declined 2.7 percentage points, to 14.9 percent overall, and it fell for virtually all demographic groups. Median holdings fell 9.1 percent, but the mean rose 4.8 percent.

Other bond types tend to be very narrowly held, and the ownership rate fell to 1.6 percent in 2007 , a drop of 0.2 percentage point from 2004.22 Although the ownership rate for such bonds fell only slightly, changes in the types of bonds held by families were somewhat larger and were driven mainly by a decline

22. "Other bonds" as reported in the survey are held directly and include corporate and mortgage-backed bonds; federal, state, and local government bonds; and foreign bonds. In this article, financial assets held indirectly are those held in retirement accounts or in other managed assets. in the fraction of families owning bonds of multiple types. The proportion of families that owned government bills and bonds, mortgage-backed bonds, and corporate or foreign bonds fell in the recent period, while ownership of tax-exempt bonds was unchanged (table 6.2).

6.2.

\begin{tabular}{c|c|c}
\hline \multirow{2}{*}{ Type of bond } & \multicolumn{2}{|c}{ All families } \\
\cline { 2 - 3 } & $\begin{array}{c}2007 \\
\text { (percent) }\end{array}$ & $\begin{array}{c}\text { Change, 2004-07 } \\
\text { (percentage points) }\end{array}$ \\
\hline Government $\ldots \ldots \ldots \ldots \ldots \ldots \ldots \ldots \ldots \ldots \ldots$ & .4 & -.1 \\
Tax exempt $\ldots \ldots \ldots \ldots \ldots \ldots$ & 1.0 & -.1 \\
Mortgage backed $\ldots \ldots \ldots \ldots \ldots$ & .3 & -.4 \\
Corporate or foreign $\ldots \ldots \ldots \ldots$ & .4 & \\
\hline
\end{tabular}

† Less than 0.05 percent.

Ownership of any type of bond is concentrated among the highest tiers of the income and wealth distributions, and these groups saw little change in ownership from 2004 to 2007. The median value of bonds for families that had them rose 12.0 percent, while the mean fell 4.4 percent.

\section{Publicly Traded Stock}

The direct ownership of publicly traded stocks is more widespread than the direct ownership of bonds, but, as with bonds, it is also concentrated among high-income and high-wealth families. The share of families with any such stock holdings declined 2.8 percentage points from 2004 to 2007, to 17.9 percent, thereby continuing a decline observed over the previous three-year period. Across demographic groups, the recent decline was most marked for the highest decile of the income distribution, families headed by a person who was aged 55 to 74 or who was self-employed, families in the Northeast or the Midwest, and families in the top quartile of the net worth distribution.

The major stock price indexes increased about 30 percent over the 2004-07 period; at the same time, the median amount of directly held stock for families with such assets rose 3.0 percent, and the mean climbed 25.6 percent. The median value declined for many demographic groups but rose substantially for the two family-structure groups with children and for the self-employed. The mean amount of directly held stock increased across most demographic groups (data not shown in the tables).

The great majority of families with directly held stock owned stock in only a small number of companies. Over the three-year period, the share of families owning stock in only one company increased (table 6.3). 
6.3.

\begin{tabular}{c|c|c}
\hline \multirow{2}{*}{$\begin{array}{c}\text { Number of } \\
\text { directly held stocks }\end{array}$} & \multicolumn{2}{|c}{ Families with directly held stocks } \\
\cline { 2 - 3 } & $\begin{array}{c}2007 \\
\text { (percent) }\end{array}$ & $\begin{array}{c}\text { Change, 2004-07 } \\
\text { (percentage points) }\end{array}$ \\
\hline $1 \ldots \ldots \ldots \ldots \ldots \ldots \ldots \ldots \ldots \ldots$ & 36.4 & 1.8 \\
2 to $9 \ldots \ldots \ldots \ldots \ldots \ldots \ldots \ldots$ & -.1 \\
10 or more $\ldots \ldots \ldots \ldots \ldots \ldots$ & 16.6 & -1.7 \\
\hline
\end{tabular}

For 36.1 percent of stockowners in 2007, at least one of the companies in which they owned stock was one that employed, or had employed, the family head or that person's spouse or partner. Ownership of stock in a foreign company was less common; only 15.8 percent of stockholders had this type of stock (data not shown in the tables). The 2004 data show a similar pattern.

\section{Pooled Investment Funds}

Pooled investment funds are among the least commonly held of the specific financial assets shown in table $6 .{ }^{23}$ As was the case for directly held bonds and stocks from 2004 to 2007, direct ownership of pooled investment funds fell-a decline of 3.6 percentage points, to 11.4 percent of families in 2007. Ownership of pooled investment funds declined for almost every demographic group over the three-year period. Both the overall change and the changes for demographic groups continue the pattern observed in the previous three-year period.

The survey also collects information on the different types of pooled investment funds owned by families. Ownership shifted over the recent period to stock funds from most other types of funds; the residual "other" category, which consists almost entirely of hedge funds and exchange-traded funds, decreased slightly (table 6.4).

Among families owning pooled investment funds, the value of holdings has continued an increase seen over the preceding decade; in the recent three-year period, the median holding rose 26.1 percent, and the mean rose 53.3 percent. Median and mean values increased across almost every demographic group, evidence that the decrease in ownership was concentrated among families with small account balances (data not shown in the tables).

23. In this article, pooled investment funds exclude money market mutual funds and indirectly held mutual funds and include all other types of directly held pooled investment funds, such as traditional open-end and closed-end mutual funds, real estate investment trusts, and hedge funds.
6.4 .

\begin{tabular}{|c|c|c|}
\hline \multirow{2}{*}{$\begin{array}{l}\text { Type of pooled } \\
\text { investment fund }\end{array}$} & \multicolumn{2}{|c|}{ All families } \\
\hline & $\begin{array}{c}2007 \\
\text { (percent) }\end{array}$ & $\begin{array}{l}\text { Change, 2004-07 } \\
\text { (percentage points) }\end{array}$ \\
\hline Stock $\ldots . . . \ldots$ & 10.2 & 3.2 \\
\hline Tax-free bond $\ldots$ & 2.1 & -.8 \\
\hline Government bond & 1.2 & .1 \\
\hline Other bond ........ & 1.0 & -.5 \\
\hline Combination .. & 1.4 & -1.3 \\
\hline Other $\ldots \ldots \ldots$. & .5 & -.2 \\
\hline
\end{tabular}

\section{Retirement Accounts}

Ownership of tax-deferred retirement assets such as personally established individual retirement accounts (IRAs) or job-based 401(k) accounts tends to increase with families' income and net worth. ${ }^{24}$ For several reasons, ownership is also more likely among families headed by a person less than 65 years of age than among the older groups. First, even though retirement accounts have been in existence for more than 25 years, they may not have become common until relatively late in the careers of many persons in the older groups. Second, beginning in the year that a person reaches age 591/2, funds held by that person in retirement accounts may be withdrawn without penalty, and some in the two oldest age groups may have already done so. Third, families may have used funds from retirement accounts accumulated from previous employment to purchase an annuity at retirement; annuities are treated in the SCF as a separate type of managed asset.

From 2004 to 2007, the fraction of families with retirement accounts rose 2.9 percentage points, to 52.6 percent; the increase offset most of the 3.0 percentage point decrease over the preceding three years. In the recent period, the fraction of families that had some type of account plan associated with a current or past job or that held an IRA or Keogh account

24. Tax-deferred retirement accounts consist of IRAs, Keogh accounts, and certain employer-sponsored accounts. Employersponsored accounts consist of 401(k), 403(b), and thrift savings accounts from current or past jobs; other current job plans from which loans or withdrawals can be made; and accounts from past jobs from which the family expects to receive the account balance in the future. This definition of employer-sponsored plans is intended to confine the analysis to accounts that are portable across jobs and for which families will ultimately have the option to withdraw the balance.

IRAs and Keoghs may be invested in virtually any asset, including stocks, bonds, pooled investment funds, options, and real estate. In principle, employer-sponsored plans may be invested in a similarly broad way, but, in practice, a person's choices for investment are sometimes limited to a narrower set of assets. 
increased, and the fraction that had at least one account of each type rose as well (table 6.5).

6.5 .

\begin{tabular}{c|c|c}
\hline \multirow{2}{*}{ Type of retirement account } & \multicolumn{2}{|c}{ All families } \\
\cline { 2 - 3 } & $\begin{array}{c}2007 \\
\text { (percent) }\end{array}$ & $\begin{array}{c}\text { Change, 2004-07 } \\
\text { (percentage points) }\end{array}$ \\
\hline $\begin{array}{c}\text { Account plan from current } \\
\text { or past job ............ } \\
\text { Individual retirement account } \\
\text { or Keogh ............... }\end{array}$ & 38.0 & 2.0 \\
$\begin{array}{l}\text { Memo } \\
\text { Both types } \ldots \ldots \ldots \ldots \ldots \ldots\end{array}$ & 14.3 & 1.6 \\
\hline
\end{tabular}

Over the 2004-07 period, ownership increased for nearly all groups. Substantial increases were reported for families in the 45-to-54 and 65-to-74 age groups, nonwhite or Hispanic families, families living in the South, and families in the technical, sales, or services occupation group.

In a continuation of the trend over the preceding decade, holdings in retirement accounts increased markedly in the 2004-07 period; for families having retirement accounts, the median rose 16.3 percent, and the mean rose 9.5 percent. Gains also appeared in the median holdings of most demographic groups over the recent period; some of the largest increases were for families in the middle of the income and wealth distributions, families in the high-schooldiploma and some-college education groups, single families with children, nonwhite or Hispanic families, the self-employed work-status group, families in the South and West, and families residing in non-MSA areas.

Although tax-deferred retirement assets are clearly an important element in retirement planning, families may hold a variety of other assets that are intended, at least in part, to finance retirement. Such other assets might also be used for contingencies as necessary. Similarly, a need for liquidity might drive a family to liquidate or borrow against a tax-deferred retirement asset, even if it will be assessed a penalty for doing so.

Two common and often particularly important types of retirement plans are not included in the assets described in this section: Social Security (the federally funded Old-Age and Survivors' Insurance program, or OASI) and employer-sponsored definedbenefit plans. OASI is well described elsewhere, and it covers the great majority of the population. ${ }^{25}$ The retirement income provided by defined-benefit plans

25. For a detailed description of OASI, see Social Security Administration, "Online Social Security Handbook," Publication 65-008, www.ssa.gov/OP_Home/handbook/ssa-hbk.htm. is typically based on workers' salaries and years of work with an employer, a group of employers, or a union. Unfortunately, future income streams from OASI and defined-benefit plans cannot be translated directly into a current value because valuation depends critically on assumptions about future events and conditions-work decisions, earnings, inflation rates, discount rates, mortality, and so on-and no widely agreed-upon standards exist for making these assumptions. ${ }^{26}$

However, the SCF does contain substantial information for family heads and their spouse or partner regarding any defined-benefit plans or other types of plans with some kind of account feature to which they have rights from a current or past job. ${ }^{27}$ In 2007, 57.7 percent of families had rights to some type of plan other than OASI through the current or past work of either the family head or that person's spouse or partner, a level nearly the same as in 2004. For this group of families, the fraction with a standard definedbenefit plan with an annuity payout scheme declined over the recent period, while the fraction with a plan with at least some account feature and the fraction that had both types of plans increased (table 6.6).

6.6.

\begin{tabular}{c|c|c}
\hline \multirow{2}{*}{ Type of pension plan } & \multicolumn{2}{|c}{ Families with any pension plan } \\
\cline { 2 - 3 } & $\begin{array}{c}2007 \\
\text { (percent) }\end{array}$ & $\begin{array}{c}\text { Change, 2004-07 } \\
\text { (percentage points) }\end{array}$ \\
\hline $\begin{array}{l}\text { Defined benefit } \ldots \ldots \ldots \ldots \ldots . \\
\text { Account plan } \ldots \ldots \ldots \ldots \ldots \ldots\end{array}$ & 55.8 & -1.6 \\
Memo & 65.8 & 3.3 \\
Both types $\ldots \ldots \ldots \ldots \ldots \ldots$ & 21.6 & 1.8 \\
\hline
\end{tabular}

In many pension plans with account features, contributions may be made by the employer, the worker, or both. In some cases, these contributions represent a substantial amount of saving, though workers may offset this saving by reducing their saving in other forms. An employer's contributions also represent additional income for the worker. In 2007, 87.1 percent of families with an account plan on a current job

26. For one possible calculation of net worth that includes the annuity value of payments from defined-benefit pensions and OASI, see Arthur B. Kennickell and Annika E. Sundén (1997), "Pensions, Social Security, and the Distribution of Wealth," Finance and Economics Discussion Series 1997-55 (Washington: Board of Governors of the Federal Reserve System, October), www.federalreserve.gov/ pubs/ feds/1997/index.html.

27. The definition of account plan used here differs slightly from that used in computing the survey wealth measure, which includes account balances only if the family has the ability to make withdrawals from, or borrow against, the account. Here the only criterion used in classification is whether any account balance exists. For example, a defined-benefit plan with a portable cash option, which would allow the covered worker to receive a lump sum in lieu of regular payments in retirement, would be treated as an account plan here. 
of either the family head or that person's spouse or partner had an employer that made contributions to the plan, a decline of 1.6 percentage points from 2004. In 2007, 91.4 percent of families with such plans made contributions themselves, an increase of 2.1 percentage points from 2004. The median annual contribution by employers who contributed to such accounts was $\$ 2,200$ in 2007 , and the median contribution by families that contributed was $\$ 2,500$; both amounts fell slightly from 2004 levels (data not shown in the tables).

The eligibility of working heads of families to participate in any type of job-related pension rose from 54.8 percent in 2004 to 55.9 percent in 2007; it had declined 2.4 percentage points over the preceding three years (data not shown in the tables). Participation by eligible workers is usually voluntary. In 2007, 83.8 percent of family heads who were eligible to participate elected to do so, down slightly from 84.1 percent in $2004 .^{28}$ The choice to participate appears to be related strongly to income. In 2007, the fraction of eligible family heads declining to participate fell as income rose, and this general pattern was not substantially altered from 2004 (table 6.7).

6.7.

\begin{tabular}{|c|c|c|}
\hline \multirow[t]{2}{*}{ Percentile of income } & \multicolumn{2}{|c|}{$\begin{array}{l}\text { Families headed by a person who } \\
\text { was eligible for a work-related } \\
\text { retirement plan on a current job and } \\
\text { who declined to participate }\end{array}$} \\
\hline & $\begin{array}{c}2007 \\
\text { (percent) }\end{array}$ & $\begin{array}{l}\text { Change, 2004-07 } \\
\text { (percentage points) }\end{array}$ \\
\hline Less than 20 & 54.3 & 3.7 \\
\hline $20-39.9 \ldots \ldots$ & 28.1 & -1.6 \\
\hline $40-59.9 \ldots \ldots$ & 18.5 & .3 \\
\hline $60-79.9$ & 10.5 & -1.5 \\
\hline $80-89.9$ & 10.9 & 2.0 \\
\hline $90-100 \quad \ldots$ & 6.5 & 1.5 \\
\hline
\end{tabular}

Cash Value Life Insurance

Cash value life insurance combines an investment vehicle with insurance coverage in the form of a death benefit. ${ }^{29}$ Some cash value life insurance policies offer a high degree of choice in the way the

28. An analysis of the March Current Population Survey (CPS) with a definition of family head that is closest to that in this article shows an opposite trend in pension eligibility for employed family heads, but that trend is at a similar level as in the SCF. The CPS eligibility estimate for family heads with a job in the past year was 57.8 percent in 2004 and 53.9 percent in 2007. Differences in the definition of the relevant employment may explain some of the difference in the levels in the two surveys. Unlike the SCF, the CPS shows a small increase in the uptake rate for such eligible workersfrom 83.0 percent in 2004 to 83.3 percent in 2007 .

29. The survey measures the value of such policies according to their current cash value, not their death benefit. The cash value is included as an asset in this article only when the cash value at the time of the interview was nonzero. policy payments are invested. Investment returns on such policies are typically shielded from taxation until the money is withdrawn; if the funds remain untapped until the policyholder dies, the beneficiary of the policy may receive, tax-free, the death benefit or the cash value, whichever is greater. In contrast, term insurance, the other popular type of life insurance, offers only a death benefit. One attraction of cash value policies for some people is that they promote regular saving funded through the required policy premium.

Ownership of cash value life insurance is broadly spread across demographic groups, with a tendency toward increasing rates among families with higher levels of income and net worth and those with older family heads. Ownership of cash value policies over the 2004-07 period continued a declining trend, decreasing 1.2 percentage points, to 23.0 percent of families in 2007. The decline was shared by most demographic groups. Over the three-year period, ownership of any type of life insurance, cash value or term, also fell slightly_from 65.4 percent in 2004 to 64.9 percent in 2007 (data not shown in the tables). Of those families with some type of life insurance, the proportion with term policies was about unchanged, while the proportion with cash value policies fell; these changes are similar to trends in the earlier surveys.

After declining over the previous three-year period, the median value of cash value life insurance for families that had any such insurance rose 21.2 percent between 2004 and 2007, and the mean rose 23.7 percent. The median showed increases across most demographic groups, although it declined considerably for families in the other-not-working work-status category, renter families, and families in the second quartile of the wealth distribution.

\section{Other Managed Assets}

Ownership of other managed assets-personal annuities and trusts with an equity interest and managed investment accounts-is concentrated among families with higher levels of income and wealth and among families headed by a person who is aged 55 or older or who is retired. ${ }^{30}$ Ownership of these assets

\footnotetext{
30. Annuities may be those in which the family has an equity interest in the asset or in which the family possesses an entitlement only to a stream of income. The wealth figures in this article include only the annuities in which the family has an equity interest. In 2007, 5.5 percent of families reported having any type of annuity, and of these families, 81.0 percent reported having an equity interest. The trusts or managed investment accounts included in other managed assets are those in which families have an equity interest and for which component parts were not separately reported. Typically, such accounts
} 
declined 1.5 percentage points between 2004 and 2007 after a small increase over the previous three years. Ownership fell in the recent three-year period for almost every demographic group, with the largest declines for families in the Midwest and for the next-to-highest income and net worth groups. Across all families, the fraction with an annuity declined over the period, and the fraction with a trust or managed investment account inched up, while the fraction with both categories of managed assets was essentially unchanged (table 6.8).

6.8.

\begin{tabular}{l|c|c}
\hline \multirow{2}{*}{ Type of other managed asset } & \multicolumn{2}{|c}{ All families } \\
\cline { 2 - 3 } & $\begin{array}{c}2007 \\
\text { (percent) }\end{array}$ & $\begin{array}{c}\text { Change, 2004-07 } \\
\text { (percentage points) }\end{array}$ \\
\hline $\begin{array}{r}\text { Annuity ................ } \\
\text { Trust or managed investment } \\
\text { account ............... }\end{array}$ & 4.5 & -1.4 \\
$\begin{array}{l}\text { Memo } \\
\text { Both types ............... }\end{array}$ & \multicolumn{2}{|}{.1} \\
\hline
\end{tabular}

$\dagger$ Less than 0.05 percent.

Between 2004 and 2007, the median value of other managed assets for families that had such assets increased 41.7 percent, an increase that offset the decline in the preceding three-year period. Over the more recent period, the corresponding mean value increased 9.4 percent. Median holdings rose for many demographic groups; noticeable exceptions were families in the top two income deciles and families headed by a person who was working for someone else or who was working in a technical, sales, or service job or a job in the other-occupation category. The rise in the median value reflects substantial increases in annuities and modest increases in trusts or managed investment accounts. For families with an equity interest in an annuity, the median holding rose 23.1 percent, to $\$ 50,000$ in 2007 ; for families with a trust or managed investment account as defined in

are those in which the ownership is complicated or the management is undertaken by a professional. In $2007,84.8$ percent of families with trusts or managed investment accounts had an equity interest in such an account.

The survey encourages respondents who have trusts or managed investment accounts that are held in relatively common investments to report the components. Of the 3.8 percent of families that reported having any kind of trust or managed investment account in 2007, 47.1 percent of them reported at least one of the component assets separately. Of families that detailed the components in 2007, 84.8 percent reported some type of financial asset, 19.0 percent reported a primary residence, 15.3 percent reported other real estate, 15.3 percent reported a business, and 2.9 percent reported another type of asset (data not shown in the tables). The fraction of these families reporting the primary residence as a trust component increased 8.0 percentage points between 2004 and 2007, and the fraction reporting a business increased 11.7 percentage points; the fraction reporting other real estate or another type of asset was little changed. this article, the median holding rose 9.1 percent, to $\$ 120,000$ (data not shown in the tables).

As noted in the discussion of retirement accounts, some families use settlements from retirement accounts to purchase an annuity. In 2007, 30.4 percent of families with annuities had done so (data not shown in the tables). Of these families, 71.7 percent had an equity interest in their annuities.

\section{Other Financial Assets}

Ownership of other financial assets-a heterogeneous category including oil and gas leases, futures contracts, royalties, proceeds from lawsuits or estates in settlement, and loans made to others-fell 0.7 percentage point between 2004 and 2007, to 9.3 percent. Ownership of such assets tends to be more common among higher income and wealth groups, younger age groups, and families headed by a person who is self-employed. Ownership across demographic groups generally declined over this period, while the median holding for those who had such assets increased 36.4 percent, to $\$ 6,000$.

Holdings may be grouped into four categories: cash, which includes money owed to families by other persons; future proceeds, which include amounts to be received from a lawsuit, estate, or other type of settlement; business items, which include deferred compensation, royalties, futures contracts, and derivatives; and other. The proportion of families holding various types of other financial assets remained fairly constant over the three-year period, with cash being by far the most frequently held component (table 6.9).

6.9.

\begin{tabular}{c|c|c}
\hline \multirow{2}{*}{ Type of other financial asset } & \multicolumn{2}{|c}{ All families } \\
\cline { 2 - 3 } & $\begin{array}{c}2007 \\
\text { (percent) }\end{array}$ & $\begin{array}{c}\text { Change, 2004-07 } \\
\text { (percentage points) }\end{array}$ \\
\hline Cash $\ldots \ldots \ldots \ldots \ldots \ldots \ldots \ldots \ldots$ & 8.1 & -.8 \\
Future proceeds $\ldots \ldots \ldots \ldots \ldots \ldots$ & .9 & .1 \\
Business items $\ldots \ldots \ldots \ldots \ldots \ldots$ & .5 & .1 \\
Other $\ldots \ldots \ldots \ldots \ldots \ldots \ldots \ldots$ & $\dagger$ & $\dagger$ \\
\hline
\end{tabular}

$\dagger$ Less than 0.05 percent.

Some publicly traded companies offer stock options to their employees as a form of compensation. ${ }^{31}$ Although stock options, when executed, may represent an appreciable part of a family's net worth, the survey does not specifically ask for the value of these options. ${ }^{32}$ Instead, the survey asks whether the family

31. See Jeffrey L. Schildkraut (2004), "Stock Options: National Compensation Survey Update" (Washington: Bureau of Labor Statistics, September), www.bls.gov/opub/cwc/cm20040628yb01p1.htm.

32. Because such options are typically not publicly traded or their execution is otherwise constrained, their value is uncertain until the 
7. Direct and indirect family holdings of stock, by selected characteristics of families, 1998-2007 surveys Percent except as noted

\begin{tabular}{|c|c|c|c|c|c|c|c|c|c|c|c|c|}
\hline \multirow{2}{*}{ Family characteristic } & \multicolumn{4}{|c|}{$\begin{array}{l}\text { Families having stock holdings, } \\
\text { direct or indirect }\end{array}$} & \multicolumn{4}{|c|}{$\begin{array}{l}\text { Median value among families with holdings } \\
\text { (thousands of } 2007 \text { dollars) }\end{array}$} & \multicolumn{4}{|c|}{$\begin{array}{l}\text { Stock holdings as share of } \\
\text { group's financial assets }\end{array}$} \\
\hline & 1998 & 2001 & 2004 & 2007 & 1998 & 2001 & 2004 & 2007 & 1998 & 2001 & 2004 & 2007 \\
\hline All families & 48.9 & 52.2 & 50.2 & 51.1 & 31.8 & 40.4 & 35.7 & 35.0 & 54.0 & 56.1 & 51.3 & 53.3 \\
\hline Percentile of income & & & & & & & & & & & & \\
\hline Less than $20 . . . .$. & 10.0 & 12.9 & 11.7 & 13.6 & 6.4 & 8.8 & 8.2 & 6.5 & 20.4 & 37.4 & 32.0 & 39.0 \\
\hline $20-39.9 \ldots \ldots \ldots \ldots$ & 30.8 & 34.1 & 29.6 & 34.0 & 12.7 & 9.1 & 11.0 & 8.8 & 29.8 & 35.6 & 30.9 & 34.3 \\
\hline $40-59.9$ & 50.2 & 52.5 & 51.7 & 49.5 & 15.3 & 17.5 & 16.5 & 17.7 & 38.1 & 46.8 & 43.4 & 38.3 \\
\hline $60-79.9$ & 69.3 & 75.7 & 69.9 & 70.5 & 24.2 & 33.5 & 28.7 & 34.1 & 45.8 & 52.0 & 41.7 & 52.5 \\
\hline $80-89.9 \ldots$ & 77.9 & 82.0 & 83.8 & 84.4 & 57.3 & 75.6 & 60.9 & 62.0 & 50.4 & 57.3 & 48.8 & 49.3 \\
\hline $90-100 \ldots$ & 90.4 & 89.7 & 92.7 & 91.0 & 171.9 & 289.7 & 225.2 & 219.0 & 62.5 & 60.5 & 57.5 & 57.6 \\
\hline Age of head (years) & & & & & & & & & & & & \\
\hline Less than $35 \ldots .$. & 40.8 & 49.0 & 40.8 & 38.6 & 8.9 & 8.2 & 8.8 & 7.0 & 44.9 & 52.5 & 40.3 & 44.3 \\
\hline $35-44 \ldots \ldots \ldots$ & 56.7 & 59.5 & 54.5 & 53.5 & 25.5 & 32.2 & 22.0 & 26.0 & 55.0 & 57.2 & 53.5 & 53.7 \\
\hline $45-54 \ldots \ldots$ & 58.6 & 59.3 & 56.5 & 60.4 & 48.4 & 58.5 & 54.9 & 45.0 & 55.7 & 59.1 & 53.8 & 53.0 \\
\hline $55-64$ & 55.9 & 57.4 & 62.8 & 58.9 & 59.8 & 94.2 & 78.0 & 78.0 & 58.4 & 56.2 & 55.0 & 55.0 \\
\hline $65-74 \ldots \ldots$ & 42.7 & 40.0 & 46.9 & 52.1 & 71.3 & 175.8 & 76.9 & 57.0 & 51.3 & 55.4 & 51.5 & 55.3 \\
\hline 75 or more .... & 29.4 & 35.7 & 34.8 & 40.1 & 76.4 & 128.7 & 94.3 & 41.0 & 48.7 & 51.8 & 39.3 & 48.1 \\
\hline Housing status & & & & & & & & & & & & \\
\hline Owner ......... & 59.8 & 62.4 & 60.9 & 62.5 & 43.3 & 58.5 & 49.4 & 41.2 & 55.1 & 56.8 & 51.9 & 53.8 \\
\hline Renter or other & 27.5 & 30.9 & 26.4 & 26.0 & 9.5 & 8.2 & 9.6 & 8.6 & 40.5 & 46.2 & 39.2 & 45.0 \\
\hline
\end{tabular}

NoTE: Indirect holdings are those in pooled investment trusts, retirement accounts, and other managed assets. See also note to table 1.

head or that person's spouse or partner had been given stock options by an employer during the preceding year. In 2007, 8.3 percent of families reported having received stock options, a decline of 1.0 percentage point below the level in 2004; this decrease continues a downward trend since the peak of 11.4 percent recorded in the SCF in 2001 (data not shown in the tables). ${ }^{33}$

\section{Direct and Indirect Holdings of Publicly Traded Stocks}

Families may hold stocks in publicly traded companies directly or indirectly, and information about each of these forms of ownership is collected separately in the SCF. When direct and indirect forms are combined, the 2007 data show a resumption of a trend of increasing stock ownership (table 7). Between 2004 and 2007, the fraction of families holding any such stock rose 0.9 percentage point, to 51.1 percent, a level still below the 2001 peak of 52.2 percent. Much like ownership of directly held stock, ownership of direct and indirect equity holdings is more common among higher-income groups and among families headed by a person aged 35 to 64 . Over the recent three-year period, ownership increased for all income groups except the third quintile and top decile. Across age groups, ownership fell for families headed by a

exercise date; until then, meaningful valuation would require complex assumptions about the future behavior of stock prices.

33. Data on the awarding of options have been collected in the SCF since 1995. person younger than 45 or aged 55 to 64; ownership rose substantially for families headed by a person aged 45 to 54 or older than 65 .

At the same time, the overall median value of direct and indirect stock holdings dropped 2.0 percent. Changes in the median value across demographic groups were mixed, with declines more common for groups that experienced increases in ownership, an indication that most new owners had small amounts. As a proportion of financial assets, holdings rose 2.0 percentage points overall, with substantial increases for the first and fourth income quintiles and the oldest age group.

As noted earlier in the discussion on net worth, the stock markets have undergone sizable declines since the data collection for the 2007 SCF was completed. To gauge the potential effect of these changes on the median amount of equity held by families, the equity values in the survey were deflated by the ratio of the average of the Wilshire 5000 index in October 2008 to the value of the index on the day of the interview, assuming a homogeneous rate of return for all equity holders and no changes in the portfolios of families since the time of the survey. Under this scenario, the median value of equity falls 35.7 percent, from the 2007 value of $\$ 35,000$ to $\$ 22,500$ (data not shown in the tables).

Among families that held equity, either directly or indirectly, in 2007, ownership through a tax-deferred retirement account was most common, followed by direct holdings of stocks, direct holdings of pooled investment funds, and managed investment accounts 
or an equity interest in a trust or annuity. Over the 2004-07 period, ownership of tax-deferred accounts rose, while ownership of all other types of equities fell; the fraction of equity owners with multiple types also declined (table 7.1).

7.1.

\begin{tabular}{|c|c|c|}
\hline \multirow{2}{*}{$\begin{array}{l}\text { Type of direct or } \\
\text { indirect equity }\end{array}$} & \multicolumn{2}{|c|}{ Families with equity } \\
\hline & $\begin{array}{c}2007 \\
\text { (percent) }\end{array}$ & $\begin{array}{l}\text { Change, 2004-07 } \\
\text { (percentage points) }\end{array}$ \\
\hline Tax-deferred account & 83.9 & 3.3 \\
\hline Directly held stock .. & 35.1 & -6.0 \\
\hline Directly held pooled & \multirow[b]{2}{*}{21.1} & \multirow{2}{*}{-7.3} \\
\hline investment fund $\ldots \ldots \ldots \ldots$ & & \\
\hline $\begin{array}{l}\text { Managed investment account, } \\
\text { or equity interest in a trust } \\
\text { or annuity } \ldots \ldots \ldots \ldots \ldots \ldots\end{array}$ & 8.1 & -1.3 \\
\hline Мемо & \multirow[b]{2}{*}{37.3} & \multirow[b]{2}{*}{-6.7} \\
\hline Multiple types . & & \\
\hline
\end{tabular}

The distribution of amounts of holdings over these types of equities shows a different pattern. Of the total amount of equity, 37.8 percent was held in taxdeferred retirement accounts, 33.6 percent as directly held stocks, 22.1 percent as directly held pooled investment funds, and 6.5 percent as other managed assets (data not shown in the tables).

\section{Nonfinancial Assets}

By definition, a rise in nonfinancial assets as a share of total assets must exactly offset the 1.8 percentage point drop in the share of financial assets from 2004 to 2007 , which was discussed earlier in this article (table 5). The changes in these shares may have been driven by changes in portfolio choices, portfolio valuation, or both. The 2001 estimate of the value of nonfinancial assets as a share of total assets, at 57.8 percent, appears to be the low point since 1998 (table 8); the 2007 level of 66.1 percent is near the middle of the range over the past seven surveys (data not shown in the tables). Over the recent three-year period, the value of primary residences as a

8. Value of nonfinancial assets of all families, distributed by type of asset, 1998-2007 surveys

Percent

\begin{tabular}{|c|c|c|c|c|}
\hline Type of nonfinancial asset & 1998 & 2001 & 2004 & 2007 \\
\hline Vehicles $^{1} \ldots \ldots \ldots$. & 6.5 & 5.9 & 5.1 & 4.4 \\
\hline Primary residence $\ldots \ldots .$. & 47.0 & 46.9 & 50.3 & 48.1 \\
\hline Other residential property ........ & 8.5 & 8.1 & 9.9 & 10.7 \\
\hline Equity in nonresidential property & 7.7 & 8.2 & 7.3 & 5.8 \\
\hline Business equity . . . . . . . . . . . & 28.5 & 29.3 & 25.9 & 29.7 \\
\hline Other ........... & 1.7 & 1.6 & 1.5 & 1.3 \\
\hline Total & 100 & 100 & 100 & 100 \\
\hline MEMO & & & & \\
\hline $\begin{array}{l}\text { Nonfinancial assets as a share of } \\
\text { total assets ................... }\end{array}$ & 59.3 & 57.8 & 64.3 & 66.1 \\
\hline
\end{tabular}

Note: See note to table 1.

1. For definition, see text note 34 . share of nonfinancial assets fell 2.2 percentage points, to 48.1 percent, still above its share before 2004 . The share of equity in nonresidential property also declined. The largest offsetting increase was in the share of business equity, which rose 3.8 percentage points over the period to its highest recorded share of 29.7 percent in 2007.

In 2007, the level of ownership of nonfinancial assets was 92.0 percent of families, 0.5 percentage point lower than in 2004 (first half of tables 9.A and 9.B, next-to-last column). Across most of the demographic groups shown, the 2007 rate was 85 percent or more; exceptions were the lowest income and wealth groups, younger childless single families, families headed by a person who was neither working nor retired, renters, families headed by a person without a high school diploma, and families living in the Northeast. Over the 2004-07 period, ownership rose most for the 55-to-64 age group, families with children, nonwhite or Hispanic families, and families living in the South. Substantial declines in ownership were seen by the oldest age group, the lowest quintile of the income distribution, families without children, families headed by a retiree, and families living in the Northeast.

Over the recent period, the median holding of nonfinancial assets for families having any such assets rose 9.3 percent, and the mean increased 16.7 percent. Across demographic groups, substantial gains in the medians far outnumbered declines. The largest gains in the median value occurred for the lowest quintile of the income distribution, and smaller gains were observed in the top four deciles, with small declines for the middle groups. Median holdings also climbed substantially among families headed by a person who was not a high school graduate, the education group with the lowest ownership of such assets.

\section{Vehicles}

Vehicles continue to be the most commonly held nonfinancial asset. ${ }^{34}$ From 2004 to 2007, the share of families that owned some type of vehicle rose 0.7 percentage point, to 87.0 percent. Trends in ownership rates over the recent three years were mixed across most demographic groups. Across age groups, ownership increased for all groups except the 35-to-44 and 75-or-more age categories. Vehicle ownership de-

34. The definition of vehicles in this article is a broad one that includes cars, vans, sport utility vehicles, trucks, motor homes, recreational vehicles, motorcycles, boats, airplanes, and helicopters. Of families owning any type of vehicle in $2007,99.8$ percent had a car, van, sport utility vehicle, motorcycle, or truck. The remaining types of vehicles were held by 15.4 percent of families. 
9. Family holdings of nonfinancial assets and of any asset, by selected characteristics of families and type of asset, 2004 and 2007 surveys

A. 2004 Survey of Consumer Finances

\begin{tabular}{|c|c|c|c|c|c|c|c|c|}
\hline Family characteristic & Vehicles & $\begin{array}{l}\text { Primary } \\
\text { residence }\end{array}$ & $\begin{array}{l}\text { Other } \\
\text { residential } \\
\text { property }\end{array}$ & $\begin{array}{c}\text { Equity in } \\
\text { nonresidential } \\
\text { property }\end{array}$ & $\begin{array}{c}\text { Business } \\
\text { equity }\end{array}$ & Other & $\begin{array}{l}\text { Any } \\
\text { nonfinancial } \\
\text { asset }\end{array}$ & Any asset \\
\hline & \multicolumn{8}{|c|}{ Percentage of families holding asset } \\
\hline All families . & 86.3 & 69.1 & 12.5 & 8.3 & 11.5 & 7.8 & 92.5 & 97.9 \\
\hline 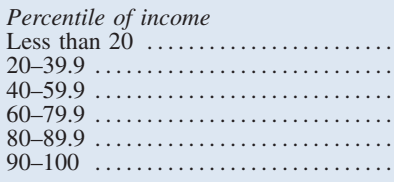 & $\begin{array}{l}65.0 \\
85.3 \\
91.6 \\
95.3 \\
95.9 \\
93.1\end{array}$ & $\begin{array}{l}40.3 \\
56.9 \\
71.6 \\
83.1 \\
91.9 \\
94.7\end{array}$ & $\begin{array}{r}3.6 \\
6.9 \\
10.0 \\
14.0 \\
19.4 \\
37.2\end{array}$ & $\begin{array}{r}2.7 \\
3.8 \\
7.6 \\
10.5 \\
12.9 \\
20.8\end{array}$ & $\begin{array}{r}3.7 \\
6.7 \\
9.5 \\
12.1 \\
15.9 \\
34.7\end{array}$ & $\begin{array}{r}3.9 \\
4.3 \\
7.6 \\
10.4 \\
8.4 \\
16.7\end{array}$ & $\begin{array}{l}76.4 \\
92.0 \\
96.7 \\
98.4 \\
99.1 \\
99.3\end{array}$ & $\begin{array}{r}92.2 \\
97.8 \\
99.8 \\
100.0 \\
99.8 \\
100.0\end{array}$ \\
\hline 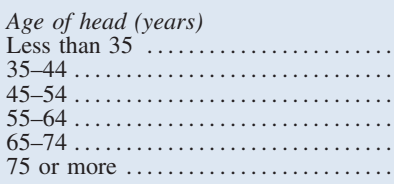 & $\begin{array}{l}82.9 \\
89.4 \\
88.8 \\
88.6 \\
89.1 \\
76.9\end{array}$ & $\begin{array}{l}41.6 \\
68.3 \\
77.3 \\
79.1 \\
81.3 \\
85.2\end{array}$ & $\begin{array}{r}5.1 \\
9.4 \\
16.3 \\
19.5 \\
19.9 \\
9.7\end{array}$ & $\begin{array}{r}3.3 \\
6.4 \\
11.4 \\
12.8 \\
10.6 \\
7.7\end{array}$ & $\begin{array}{r}6.9 \\
13.9 \\
15.7 \\
15.8 \\
8.0 \\
5.3\end{array}$ & $\begin{array}{l}5.5 \\
6.0 \\
9.7 \\
9.2 \\
9.0 \\
8.5\end{array}$ & $\begin{array}{l}88.6 \\
93.0 \\
94.7 \\
92.6 \\
95.6 \\
92.5\end{array}$ & $\begin{array}{l}96.5 \\
97.7 \\
98.3 \\
97.5 \\
99.5 \\
99.6\end{array}$ \\
\hline $\begin{array}{l}\text { Family structure } \\
\text { Single with child(ren) } \ldots \ldots \ldots \ldots \ldots \\
\text { Single, no child, age less than } 55 \ldots \ldots \\
\text { Single, no child, age } 55 \text { or more } \ldots \ldots \\
\text { Couple with child(ren) } \ldots \ldots \ldots \ldots \ldots \ldots \\
\text { Couple, no child } \ldots \ldots \ldots \ldots \ldots \ldots \ldots \ldots\end{array}$ & $\begin{array}{l}80.0 \\
77.3 \\
75.2 \\
91.3 \\
93.6\end{array}$ & $\begin{array}{l}60.6 \\
42.0 \\
70.0 \\
75.8 \\
80.1\end{array}$ & $\begin{array}{r}6.4 \\
7.1 \\
11.4 \\
14.4 \\
15.8\end{array}$ & $\begin{array}{r}5.0 \\
3.9 \\
6.8 \\
7.7 \\
11.4\end{array}$ & $\begin{array}{r}4.9 \\
7.0 \\
5.3 \\
12.2 \\
16.3\end{array}$ & $\begin{array}{l}5.9 \\
6.7 \\
7.9 \\
6.4 \\
8.8\end{array}$ & $\begin{array}{l}88.4 \\
84.1 \\
88.2 \\
95.7 \\
97.4\end{array}$ & $\begin{array}{l}96.9 \\
95.4 \\
97.8 \\
99.1 \\
98.9\end{array}$ \\
\hline $\begin{array}{l}\text { Education of head } \\
\text { No high school diploma } \ldots \ldots \ldots \ldots \ldots \\
\text { High school diploma } \ldots \ldots \ldots \ldots \ldots \ldots \\
\text { Some college } \ldots \ldots \ldots \ldots \ldots \ldots \ldots \ldots \\
\text { College degree } \ldots \ldots \ldots \ldots \ldots \ldots \ldots\end{array}$ & $\begin{array}{l}70.1 \\
87.6 \\
88.2 \\
90.7\end{array}$ & $\begin{array}{l}56.3 \\
65.8 \\
64.5 \\
79.1\end{array}$ & $\begin{array}{r}5.6 \\
8.3 \\
12.2 \\
19.0\end{array}$ & $\begin{array}{r}4.0 \\
6.1 \\
8.1 \\
11.9\end{array}$ & $\begin{array}{r}4.2 \\
10.4 \\
10.7 \\
15.6\end{array}$ & $\begin{array}{r}1.9 \\
5.3 \\
9.4 \\
11.3\end{array}$ & $\begin{array}{l}81.9 \\
92.4 \\
93.3 \\
96.5\end{array}$ & $\begin{array}{l}91.1 \\
98.1 \\
99.1 \\
99.9\end{array}$ \\
\hline $\begin{array}{l}\text { Race or ethnicity of respondent } \\
\text { White non-Hispanic } \ldots \ldots \ldots \ldots \ldots \ldots \\
\text { Nonwhite or Hispanic } \ldots \ldots \ldots \ldots \ldots \ldots\end{array}$ & $\begin{array}{l}90.3 \\
76.1\end{array}$ & $\begin{array}{l}76.1 \\
50.8\end{array}$ & $\begin{array}{r}14.0 \\
8.9\end{array}$ & $\begin{array}{l}9.2 \\
5.8\end{array}$ & $\begin{array}{r}13.6 \\
5.9\end{array}$ & $\begin{array}{l}9.3 \\
3.8\end{array}$ & $\begin{array}{l}95.8 \\
84.0\end{array}$ & $\begin{array}{l}99.3 \\
94.4\end{array}$ \\
\hline $\begin{array}{l}\text { Current work status of head } \\
\text { Working for someone else } \ldots \ldots \ldots \ldots \\
\text { Self-employed } \ldots \ldots \ldots \ldots \ldots \ldots \ldots \ldots \\
\text { Retired } \ldots \ldots \ldots \ldots \ldots \ldots \ldots \ldots \ldots \\
\text { Other not working } \ldots \ldots \ldots \ldots \ldots \ldots \ldots\end{array}$ & $\begin{array}{l}89.7 \\
91.2 \\
79.0 \\
66.9\end{array}$ & $\begin{array}{l}66.5 \\
79.1 \\
75.8 \\
40.0\end{array}$ & $\begin{array}{r}10.4 \\
25.8 \\
12.8 \\
5.4\end{array}$ & $\begin{array}{r}6.8 \\
18.7 \\
7.9 \\
*\end{array}$ & $\begin{array}{r}5.8 \\
58.1 \\
3.5 \\
6.9\end{array}$ & $\begin{array}{r}7.1 \\
12.9 \\
7.1 \\
6.4\end{array}$ & $\begin{array}{l}93.8 \\
97.5 \\
89.8 \\
76.3\end{array}$ & $\begin{array}{l}98.4 \\
99.1 \\
97.7 \\
89.6\end{array}$ \\
\hline $\begin{array}{l}\text { Current occupation of head } \\
\text { Managerial or professional } \ldots \ldots \ldots \ldots \\
\text { Technical, sales, or services } \ldots \ldots \ldots \ldots \\
\text { Other occupation } \ldots \ldots \ldots \ldots \ldots \ldots \\
\text { Retired or other not working } \ldots \ldots \ldots \ldots\end{array}$ & $\begin{array}{l}92.0 \\
85.1 \\
92.1 \\
77.2\end{array}$ & $\begin{array}{l}78.1 \\
58.2 \\
66.6 \\
70.3\end{array}$ & $\begin{array}{r}19.6 \\
8.2 \\
9.0 \\
11.6\end{array}$ & $\begin{array}{r}11.3 \\
6.9 \\
7.4 \\
7.1\end{array}$ & $\begin{array}{r}21.2 \\
9.7 \\
10.2 \\
4.0\end{array}$ & $\begin{array}{r}10.4 \\
7.2 \\
5.9 \\
7.0\end{array}$ & $\begin{array}{l}97.0 \\
90.9 \\
94.7 \\
87.7\end{array}$ & $\begin{array}{l}99.9 \\
97.4 \\
97.8 \\
96.4\end{array}$ \\
\hline 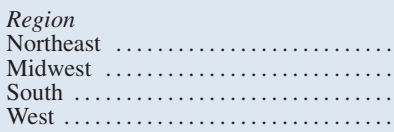 & $\begin{array}{l}80.4 \\
89.4 \\
84.9 \\
90.6\end{array}$ & $\begin{array}{l}69.8 \\
73.5 \\
68.9 \\
64.0\end{array}$ & $\begin{array}{l}12.6 \\
12.6 \\
10.2 \\
16.3\end{array}$ & $\begin{array}{l}6.0 \\
8.2 \\
8.8 \\
9.6\end{array}$ & $\begin{array}{l}11.1 \\
12.6 \\
10.1 \\
13.0\end{array}$ & $\begin{array}{l}6.4 \\
8.8 \\
7.1 \\
8.9\end{array}$ & $\begin{array}{l}90.3 \\
94.2 \\
92.1 \\
93.4\end{array}$ & $\begin{array}{l}97.9 \\
99.2 \\
97.3 \\
97.7\end{array}$ \\
\hline $\begin{array}{l}\text { Urbanicity } \\
\text { Metropolitan statistical area (MSA) } \ldots \\
\text { Non-MSA ...................... }\end{array}$ & $\begin{array}{l}85.9 \\
88.3\end{array}$ & $\begin{array}{l}68.0 \\
74.0\end{array}$ & $\begin{array}{r}13.3 \\
8.7\end{array}$ & $\begin{array}{l}8.0 \\
9.8\end{array}$ & $\begin{array}{l}11.6 \\
11.0\end{array}$ & $\begin{array}{l}8.3 \\
5.1\end{array}$ & $\begin{array}{l}92.1 \\
94.6\end{array}$ & $\begin{array}{l}97.8 \\
98.4\end{array}$ \\
\hline $\begin{array}{l}\text { Housing status } \\
\text { Owner ......... } \\
\text { Renter or other. }\end{array}$ & $\begin{array}{l}92.3 \\
73.0\end{array}$ & $\begin{array}{c}100.0 \\
*\end{array}$ & $\begin{array}{r}15.7 \\
5.4\end{array}$ & $\begin{array}{r}11.0 \\
2.4\end{array}$ & $\begin{array}{r}14.7 \\
4.3\end{array}$ & $\begin{array}{l}9.2 \\
4.6\end{array}$ & $\begin{array}{r}100.0 \\
75.9\end{array}$ & $\begin{array}{r}100.0 \\
93.3\end{array}$ \\
\hline 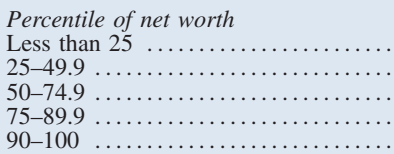 & $\begin{array}{l}69.8 \\
89.2 \\
92.0 \\
95.2 \\
93.1\end{array}$ & $\begin{array}{l}15.2 \\
71.2 \\
93.4 \\
96.2 \\
96.9\end{array}$ & $\begin{array}{c}* \\
4.9 \\
12.7 \\
23.1 \\
45.6\end{array}$ & $\begin{array}{r}* \\
4.1 \\
8.3 \\
15.1 \\
28.8\end{array}$ & $\begin{array}{r}* \\
5.6 \\
11.2 \\
19.9 \\
40.8\end{array}$ & $\begin{array}{r}2.9 \\
5.4 \\
7.8 \\
12.3 \\
18.8\end{array}$ & $\begin{array}{l}73.7 \\
97.5 \\
99.0 \\
99.8 \\
99.9\end{array}$ & $\begin{array}{r}91.7 \\
100.0 \\
100.0 \\
100.0 \\
100.0\end{array}$ \\
\hline
\end{tabular}


9. Family holdings of nonfinancial assets and of any asset, by selected characteristics of families and type of asset, 2004 and 2007 surveys-Continued

A. 2004 Survey of Consumer Finances-continued

\begin{tabular}{|c|c|c|c|c|c|c|c|c|}
\hline Family characteristic & Vehicles & $\begin{array}{l}\text { Primary } \\
\text { residence }\end{array}$ & $\begin{array}{l}\text { Other } \\
\text { residential } \\
\text { property }\end{array}$ & $\begin{array}{c}\text { Equity in } \\
\text { nonresidential } \\
\text { property }\end{array}$ & $\begin{array}{c}\text { Business } \\
\text { equity }\end{array}$ & Other & $\begin{array}{c}\text { Any } \\
\text { nonfinancial } \\
\text { asset }\end{array}$ & Any asset \\
\hline & \multicolumn{8}{|c|}{ Median value of holdings for families holding asset (thousands of 2007 dollars) } \\
\hline All families . & 15.6 & 175.7 & 109.8 & 65.9 & 109.8 & 16.5 & 162.3 & 189.9 \\
\hline 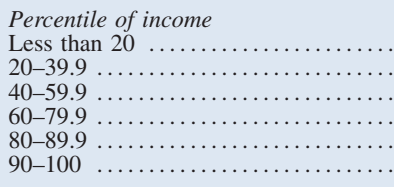 & $\begin{array}{r}5.0 \\
8.5 \\
14.4 \\
21.8 \\
28.3 \\
36.2\end{array}$ & $\begin{array}{r}76.9 \\
109.8 \\
148.3 \\
192.2 \\
247.1 \\
494.2\end{array}$ & $\begin{array}{r}36.2 \\
71.4 \\
60.4 \\
109.8 \\
107.6 \\
286.9\end{array}$ & $\begin{array}{r}12.1 \\
32.9 \\
39.5 \\
47.2 \\
65.9 \\
207.6\end{array}$ & $\begin{array}{r}32.9 \\
32.9 \\
68.6 \\
164.7 \\
109.8 \\
384.4\end{array}$ & $\begin{array}{r}4.9 \\
7.7 \\
11.0 \\
11.0 \\
19.2 \\
54.9\end{array}$ & $\begin{array}{r}24.6 \\
78.0 \\
145.3 \\
216.5 \\
309.5 \\
715.2\end{array}$ & $\begin{array}{r}18.7 \\
85.9 \\
169.7 \\
317.6 \\
503.6 \\
1,271.5\end{array}$ \\
\hline 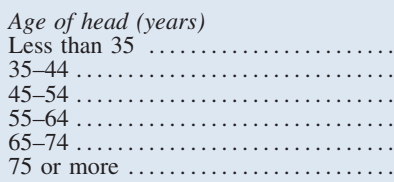 & $\begin{array}{r}12.4 \\
17.2 \\
20.6 \\
20.5 \\
13.6 \\
9.2\end{array}$ & $\begin{array}{l}148.3 \\
175.7 \\
186.7 \\
219.7 \\
164.7 \\
137.3\end{array}$ & $\begin{array}{r}90.6 \\
87.9 \\
98.8 \\
148.3 \\
87.9 \\
164.7\end{array}$ & $\begin{array}{l}60.4 \\
46.3 \\
47.2 \\
82.4 \\
85.7 \\
94.3\end{array}$ & $\begin{array}{r}54.9 \\
109.8 \\
158.2 \\
209.7 \\
109.8 \\
88.2\end{array}$ & $\begin{array}{r}5.5 \\
11.0 \\
22.0 \\
27.5 \\
32.9 \\
12.1\end{array}$ & $\begin{array}{r}35.5 \\
166.2 \\
202.6 \\
248.6 \\
177.0 \\
150.6\end{array}$ & $\begin{array}{r}43.0 \\
190.4 \\
258.0 \\
385.7 \\
256.1 \\
203.4\end{array}$ \\
\hline $\begin{array}{l}\text { Family structure } \\
\text { Single with child(ren) } \ldots \ldots \ldots \ldots \ldots \\
\text { Single, no child, age less than } 55 \ldots \ldots \\
\text { Single, no child, age } 55 \text { or more } \ldots \ldots \\
\text { Couple with child(ren) } \ldots \ldots \ldots \ldots \ldots \ldots \\
\text { Couple, no child } \ldots \ldots \ldots \ldots \ldots \ldots \ldots \ldots\end{array}$ & $\begin{array}{r}8.7 \\
9.4 \\
7.2 \\
20.9 \\
21.6\end{array}$ & $\begin{array}{l}131.8 \\
137.3 \\
130.9 \\
175.7 \\
214.2\end{array}$ & $\begin{array}{r}27.5 \\
87.9 \\
93.4 \\
98.8 \\
126.3\end{array}$ & $\begin{array}{l}15.5 \\
61.5 \\
90.1 \\
71.7 \\
68.9\end{array}$ & $\begin{array}{r}55.9 \\
63.9 \\
115.3 \\
109.8 \\
154.6\end{array}$ & $\begin{array}{l}11.0 \\
11.0 \\
11.0 \\
22.0 \\
22.0\end{array}$ & $\begin{array}{r}98.9 \\
43.9 \\
117.5 \\
195.4 \\
224.6\end{array}$ & $\begin{array}{r}95.9 \\
47.5 \\
154.2 \\
250.4 \\
314.2\end{array}$ \\
\hline $\begin{array}{l}\text { Education of head } \\
\text { No high school diploma } \ldots \ldots \ldots \ldots \\
\text { High school diploma } \ldots \ldots \ldots \ldots \ldots \\
\text { Some college } \ldots \ldots \ldots \ldots \ldots \ldots \ldots \ldots \\
\text { College degree } \ldots \ldots \ldots \ldots \ldots \ldots \ldots\end{array}$ & $\begin{array}{r}8.2 \\
13.6 \\
14.5 \\
20.7\end{array}$ & $\begin{array}{r}82.4 \\
137.3 \\
169.1 \\
263.6\end{array}$ & $\begin{array}{r}94.5 \\
76.9 \\
87.9 \\
159.3\end{array}$ & $\begin{array}{r}17.6 \\
27.5 \\
101.0 \\
87.9\end{array}$ & $\begin{array}{r}60.4 \\
88.5 \\
164.7 \\
164.7\end{array}$ & $\begin{array}{r}5.5 \\
11.0 \\
11.0 \\
22.0\end{array}$ & $\begin{array}{r}59.9 \\
119.9 \\
150.9 \\
264.9\end{array}$ & $\begin{array}{r}54.8 \\
146.5 \\
165.3 \\
392.1\end{array}$ \\
\hline $\begin{array}{l}\text { Race or ethnicity of respondent } \\
\text { White non-Hispanic } \ldots \ldots \ldots \ldots \ldots \ldots \\
\text { Nonwhite or Hispanic } \ldots \ldots \ldots \ldots \ldots \ldots\end{array}$ & $\begin{array}{l}17.3 \\
10.7\end{array}$ & $\begin{array}{l}181.2 \\
142.8\end{array}$ & $\begin{array}{r}115.3 \\
87.9\end{array}$ & $\begin{array}{l}72.5 \\
32.9\end{array}$ & $\begin{array}{r}148.3 \\
73.2\end{array}$ & $\begin{array}{l}18.1 \\
11.0\end{array}$ & $\begin{array}{r}181.0 \\
70.4\end{array}$ & $\begin{array}{r}246.6 \\
65.4\end{array}$ \\
\hline $\begin{array}{l}\text { Current work status of head } \\
\text { Working for someone else } \ldots \ldots \ldots \ldots \\
\text { Self-employed } \ldots \ldots \ldots \ldots \ldots \ldots \ldots \ldots \\
\text { Retired } \ldots \ldots \ldots \ldots \ldots \ldots \ldots \ldots \ldots \\
\text { Other not working } \ldots \ldots \ldots \ldots \ldots \ldots \ldots\end{array}$ & $\begin{array}{l}16.3 \\
24.1 \\
11.1 \\
11.8\end{array}$ & $\begin{array}{l}175.7 \\
272.4 \\
142.8 \\
142.8\end{array}$ & $\begin{array}{r}96.6 \\
155.4 \\
109.8 \\
94.5\end{array}$ & $\begin{array}{r}43.9 \\
137.3 \\
65.9 \\
*\end{array}$ & $\begin{array}{r}54.9 \\
191.1 \\
131.8 \\
27.5\end{array}$ & $\begin{array}{l}11.0 \\
32.9 \\
27.5 \\
22.0\end{array}$ & $\begin{array}{r}155.8 \\
368.3 \\
144.7 \\
65.9\end{array}$ & $\begin{array}{r}177.0 \\
514.3 \\
181.9 \\
33.3\end{array}$ \\
\hline $\begin{array}{l}\text { Current occupation of head } \\
\text { Managerial or professional } \ldots \ldots \ldots \ldots \\
\text { Technical, sales, or services } \ldots \ldots \ldots \ldots \\
\text { Other occupation } \ldots \ldots \ldots \ldots \ldots \ldots \ldots \\
\text { Retired or other not working } \ldots \ldots \ldots \ldots\end{array}$ & $\begin{array}{l}21.2 \\
14.0 \\
16.0 \\
11.1\end{array}$ & $\begin{array}{l}263.2 \\
164.7 \\
142.8 \\
142.8\end{array}$ & $\begin{array}{r}131.8 \\
115.3 \\
92.3 \\
103.2\end{array}$ & $\begin{array}{l}98.6 \\
65.9 \\
24.2 \\
65.9\end{array}$ & $\begin{array}{r}175.7 \\
82.4 \\
82.4 \\
109.8\end{array}$ & $\begin{array}{l}19.2 \\
11.0 \\
11.0 \\
27.5\end{array}$ & $\begin{array}{l}266.2 \\
121.9 \\
126.6 \\
139.6\end{array}$ & $\begin{array}{l}383.9 \\
125.6 \\
145.9 \\
162.9\end{array}$ \\
\hline 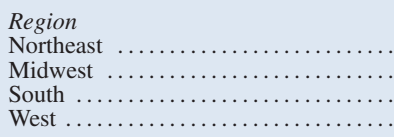 & $\begin{array}{l}17.2 \\
15.3 \\
15.1 \\
15.6\end{array}$ & $\begin{array}{l}274.6 \\
159.3 \\
142.8 \\
247.1\end{array}$ & $\begin{array}{r}115.3 \\
109.8 \\
98.8 \\
120.8\end{array}$ & $\begin{array}{r}65.9 \\
64.5 \\
38.4 \\
137.3\end{array}$ & $\begin{array}{r}109.8 \\
148.3 \\
94.9 \\
164.7\end{array}$ & $\begin{array}{l}16.5 \\
16.5 \\
16.5 \\
16.5\end{array}$ & $\begin{array}{l}228.1 \\
165.0 \\
131.4 \\
191.6\end{array}$ & $\begin{array}{l}297.1 \\
214.3 \\
145.2 \\
218.7\end{array}$ \\
\hline $\begin{array}{l}\text { Urbanicity } \\
\text { Metropolitan statistical area (MSA) } \ldots \\
\text { Non-MSA } \ldots \ldots \ldots \ldots \ldots \ldots \ldots \ldots \ldots\end{array}$ & $\begin{array}{l}15.8 \\
14.3\end{array}$ & $\begin{array}{r}197.7 \\
98.8\end{array}$ & $\begin{array}{r}120.8 \\
74.1\end{array}$ & $\begin{array}{l}76.9 \\
27.5\end{array}$ & $\begin{array}{r}118.1 \\
86.5\end{array}$ & $\begin{array}{l}16.5 \\
11.0\end{array}$ & $\begin{array}{l}178.4 \\
104.0\end{array}$ & $\begin{array}{l}218.3 \\
122.2\end{array}$ \\
\hline $\begin{array}{l}\text { Housing status } \\
\text { Owner } \ldots \ldots \ldots \ldots \ldots \ldots \ldots \ldots \ldots \ldots \ldots \ldots \ldots \ldots \ldots \ldots \ldots \\
\text { Renter or other } \ldots \ldots \ldots \ldots \ldots \ldots \ldots\end{array}$ & $\begin{array}{r}19.2 \\
7.9\end{array}$ & $\begin{array}{c}175.7 \\
*\end{array}$ & $\begin{array}{r}109.8 \\
87.9\end{array}$ & $\begin{array}{l}68.1 \\
61.5\end{array}$ & $\begin{array}{r}134.8 \\
54.9\end{array}$ & $\begin{array}{r}19.2 \\
8.8\end{array}$ & $\begin{array}{r}221.4 \\
9.2\end{array}$ & $\begin{array}{r}318.4 \\
13.4\end{array}$ \\
\hline 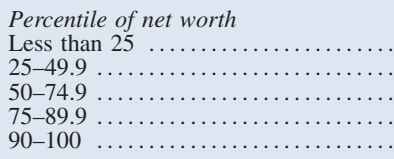 & $\begin{array}{r}6.1 \\
13.0 \\
19.1 \\
24.8 \\
33.6\end{array}$ & $\begin{array}{r}71.4 \\
93.4 \\
175.0 \\
274.6 \\
494.2\end{array}$ & \begin{tabular}{r}
\multicolumn{1}{c}{$*$} \\
28.1 \\
71.4 \\
109.8 \\
356.9
\end{tabular} & \begin{tabular}{r}
\multicolumn{1}{c}{$*$} \\
16.3 \\
27.5 \\
81.1 \\
274.6
\end{tabular} & $\begin{array}{r}* \\
19.2 \\
60.4 \\
164.7 \\
579.3\end{array}$ & $\begin{array}{r}3.3 \\
6.6 \\
11.0 \\
27.5 \\
87.9\end{array}$ & $\begin{array}{r}8.1 \\
79.5 \\
206.5 \\
396.2 \\
996.9\end{array}$ & $\begin{array}{r}8.4 \\
92.8 \\
282.5 \\
659.2 \\
1,727.1\end{array}$ \\
\hline $\begin{array}{l}\text { MEмо } \\
\text { Mean value of holdings for } \\
\text { families holding asset } \ldots\end{array}$ & 22.1 & 271.1 & 293.6 & 327.4 & 840.7 & 73.1 & 402.3 & 591.3 \\
\hline
\end{tabular}

Note: See note to table 8.

* Ten or fewer observations. 
9. Family holdings of nonfinancial assets and of any asset, by selected characteristics of families and type of asset, 2004 and 2007 surveys-Continued

B. 2007 Survey of Consumer Finances

\begin{tabular}{|c|c|c|c|c|c|c|c|c|}
\hline Family characteristic & Vehicles & $\begin{array}{c}\text { Primary } \\
\text { residence }\end{array}$ & $\begin{array}{l}\text { Other } \\
\text { residential } \\
\text { property }\end{array}$ & $\begin{array}{c}\text { Equity in } \\
\text { nonresidential } \\
\text { property }\end{array}$ & $\begin{array}{l}\text { Business } \\
\text { equity }\end{array}$ & Other & $\begin{array}{l}\text { Any } \\
\text { nonfinancial } \\
\text { asset }\end{array}$ & Any asset \\
\hline & \multicolumn{8}{|c|}{ Percentage of families holding asset } \\
\hline All families . & 87.0 & 68.6 & 13.7 & 8.1 & 12.0 & 7.2 & 92.0 & 97.7 \\
\hline 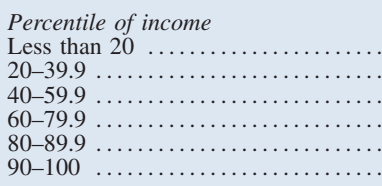 & $\begin{array}{l}64.4 \\
85.9 \\
94.3 \\
95.4 \\
95.6 \\
94.8\end{array}$ & $\begin{array}{l}41.4 \\
55.2 \\
69.3 \\
83.9 \\
92.6 \\
94.3\end{array}$ & $\begin{array}{r}5.4 \\
6.5 \\
9.9 \\
15.4 \\
21.0 \\
42.2\end{array}$ & $\begin{array}{r}2.5 \\
3.9 \\
7.4 \\
9.4 \\
13.6 \\
21.0\end{array}$ & $\begin{array}{r}3.0 \\
4.5 \\
9.2 \\
15.9 \\
17.0 \\
37.5\end{array}$ & $\begin{array}{r}3.9 \\
5.7 \\
7.4 \\
7.2 \\
9.0 \\
14.1\end{array}$ & $\begin{array}{l}73.4 \\
91.2 \\
97.2 \\
98.5 \\
99.6 \\
99.7\end{array}$ & $\begin{array}{r}89.8 \\
98.9 \\
100.0 \\
100.0 \\
100.0 \\
100.0\end{array}$ \\
\hline 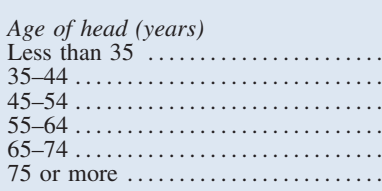 & $\begin{array}{l}85.4 \\
87.5 \\
90.3 \\
92.2 \\
90.6 \\
71.5\end{array}$ & $\begin{array}{l}40.7 \\
66.1 \\
77.3 \\
81.0 \\
85.5 \\
77.0\end{array}$ & $\begin{array}{r}5.6 \\
12.0 \\
15.7 \\
20.9 \\
18.9 \\
13.4\end{array}$ & $\begin{array}{r}3.2 \\
7.5 \\
9.5 \\
11.5 \\
12.3 \\
6.8\end{array}$ & $\begin{array}{r}6.8 \\
16.0 \\
15.2 \\
16.3 \\
10.1 \\
3.8\end{array}$ & $\begin{array}{l}5.9 \\
5.5 \\
8.7 \\
8.5 \\
9.1 \\
5.8\end{array}$ & $\begin{array}{l}88.2 \\
91.3 \\
95.0 \\
95.6 \\
94.5 \\
87.3\end{array}$ & $\begin{array}{l}97.1 \\
96.9 \\
97.6 \\
99.1 \\
98.4 \\
98.1\end{array}$ \\
\hline $\begin{array}{l}\text { Family structure } \\
\text { Single with child(ren) } \ldots \ldots \ldots \\
\text { Single, no child, age less than } 55 \ldots \\
\text { Single, no child, age } 55 \text { or more } \ldots \\
\text { Couple with child(ren) } \ldots \ldots \ldots \ldots \ldots \\
\text { Couple, no child } \ldots \ldots \ldots \ldots \ldots \ldots \ldots\end{array}$ & $\begin{array}{l}80.5 \\
77.0 \\
73.9 \\
94.0 \\
94.6\end{array}$ & $\begin{array}{l}53.4 \\
42.6 \\
68.1 \\
78.3 \\
79.2\end{array}$ & $\begin{array}{r}8.9 \\
6.2 \\
11.8 \\
14.8 \\
18.0\end{array}$ & $\begin{array}{r}5.6 \\
2.9 \\
7.3 \\
8.4 \\
10.8\end{array}$ & $\begin{array}{r}5.6 \\
7.5 \\
3.3 \\
15.6 \\
16.6\end{array}$ & $\begin{array}{l}5.8 \\
7.0 \\
5.7 \\
8.9 \\
7.4\end{array}$ & $\begin{array}{l}89.5 \\
82.5 \\
85.1 \\
96.9 \\
97.3\end{array}$ & $\begin{array}{l}95.7 \\
93.7 \\
97.7 \\
98.8 \\
99.4\end{array}$ \\
\hline $\begin{array}{l}\text { Education of head } \\
\text { No high school diploma } \ldots \ldots \ldots \ldots \\
\text { High school diploma } \ldots \ldots \ldots \ldots \ldots \ldots \\
\text { Some college } \ldots \ldots \ldots \ldots \ldots \ldots \ldots \ldots \ldots \\
\text { College degree } \ldots \ldots \ldots \ldots \ldots \ldots \ldots \ldots \ldots \ldots\end{array}$ & $\begin{array}{l}73.7 \\
87.5 \\
86.7 \\
91.9\end{array}$ & $\begin{array}{l}52.8 \\
68.9 \\
62.3 \\
77.8\end{array}$ & $\begin{array}{r}5.8 \\
10.0 \\
13.2 \\
20.6\end{array}$ & $\begin{array}{r}2.6 \\
7.3 \\
6.5 \\
11.8\end{array}$ & $\begin{array}{r}5.3 \\
8.7 \\
10.7 \\
18.2\end{array}$ & $\begin{array}{r}2.2 \\
5.1 \\
7.0 \\
11.0\end{array}$ & $\begin{array}{l}80.9 \\
92.2 \\
91.0 \\
96.6\end{array}$ & $\begin{array}{l}91.7 \\
97.7 \\
98.5 \\
99.6\end{array}$ \\
\hline $\begin{array}{l}\text { Race or ethnicity of respondent } \\
\text { White non-Hispanic } \ldots \ldots \ldots \ldots . \\
\text { Nonwhite or Hispanic } \ldots \ldots \ldots \ldots\end{array}$ & $\begin{array}{l}89.6 \\
80.9\end{array}$ & $\begin{array}{l}75.6 \\
51.9\end{array}$ & $\begin{array}{l}15.3 \\
10.0\end{array}$ & $\begin{array}{l}9.0 \\
5.9\end{array}$ & $\begin{array}{r}13.9 \\
7.4\end{array}$ & $\begin{array}{l}8.4 \\
4.3\end{array}$ & $\begin{array}{l}94.6 \\
85.8\end{array}$ & $\begin{array}{l}98.9 \\
94.9\end{array}$ \\
\hline $\begin{array}{l}\text { Current work status of head } \\
\text { Working for someone else } \ldots \ldots \\
\text { Self-employed ............... } \\
\text { Retired ..................... } \\
\text { Other not working . . . . . . . . }\end{array}$ & $\begin{array}{l}91.3 \\
90.6 \\
78.6 \\
69.3\end{array}$ & $\begin{array}{l}67.2 \\
82.4 \\
72.9 \\
33.3\end{array}$ & $\begin{array}{r}11.9 \\
26.5 \\
14.6 \\
3.8\end{array}$ & $\begin{array}{r}7.0 \\
17.3 \\
7.7 \\
4.7\end{array}$ & $\begin{array}{r}6.3 \\
68.4 \\
3.6 \\
3.6\end{array}$ & $\begin{array}{r}7.1 \\
11.0 \\
5.4 \\
8.5\end{array}$ & $\begin{array}{l}94.4 \\
97.6 \\
87.2 \\
74.8\end{array}$ & $\begin{array}{l}98.6 \\
99.7 \\
96.1 \\
90.0\end{array}$ \\
\hline $\begin{array}{l}\text { Current occupation of head } \\
\text { Managerial or professional .... } \\
\text { Technical, sales, or services .. } \\
\text { Other occupation ............ } \\
\text { Retired or other not working . }\end{array}$ & $\begin{array}{l}93.1 \\
87.4 \\
92.6 \\
77.1\end{array}$ & $\begin{array}{l}78.2 \\
61.5 \\
66.3 \\
66.7\end{array}$ & $\begin{array}{r}20.7 \\
10.2 \\
9.6 \\
12.9\end{array}$ & $\begin{array}{r}10.8 \\
7.3 \\
6.7 \\
7.2\end{array}$ & $\begin{array}{r}22.0 \\
9.2 \\
13.6 \\
3.6\end{array}$ & $\begin{array}{l}9.9 \\
7.7 \\
4.9 \\
5.9\end{array}$ & $\begin{array}{l}97.2 \\
91.6 \\
95.2 \\
85.2\end{array}$ & $\begin{array}{l}99.8 \\
97.8 \\
98.5 \\
95.2\end{array}$ \\
\hline $\begin{array}{l}\text { Region } \\
\text { Northeast } \ldots \ldots \ldots \ldots \ldots \ldots \\
\text { Midwest } \ldots \ldots \ldots \ldots \ldots \ldots \ldots \ldots \\
\text { South } \ldots \ldots \ldots \ldots \ldots \ldots \ldots \ldots \ldots \\
\text { West } \ldots \ldots \ldots \ldots \ldots \ldots \ldots \ldots\end{array}$ & $\begin{array}{l}75.4 \\
89.5 \\
89.2 \\
90.5\end{array}$ & $\begin{array}{l}66.1 \\
71.3 \\
70.2 \\
65.4\end{array}$ & $\begin{array}{l}13.3 \\
13.7 \\
11.3 \\
18.3\end{array}$ & $\begin{array}{l}5.6 \\
8.4 \\
8.8 \\
8.7\end{array}$ & $\begin{array}{r}7.8 \\
13.1 \\
11.4 \\
15.3\end{array}$ & $\begin{array}{l}5.5 \\
6.4 \\
7.2 \\
9.3\end{array}$ & $\begin{array}{l}84.2 \\
93.4 \\
93.8 \\
94.1\end{array}$ & $\begin{array}{l}94.6 \\
98.4 \\
98.5 \\
98.4\end{array}$ \\
\hline $\begin{array}{l}\text { Urbanicity } \\
\text { Metropolitan statistical area (MSA) . } \\
\text { Non-MSA .................... }\end{array}$ & $\begin{array}{l}86.2 \\
90.9\end{array}$ & $\begin{array}{l}68.1 \\
71.1\end{array}$ & $\begin{array}{l}14.2 \\
11.7\end{array}$ & $\begin{array}{r}7.6 \\
10.7\end{array}$ & $\begin{array}{l}12.3 \\
10.6\end{array}$ & $\begin{array}{l}7.6 \\
5.1\end{array}$ & $\begin{array}{l}91.5 \\
94.3\end{array}$ & $\begin{array}{l}97.7 \\
97.9\end{array}$ \\
\hline $\begin{array}{l}\text { Housing status } \\
\text { Owner } \ldots \ldots \ldots \ldots \ldots \ldots \ldots \ldots \ldots \ldots \ldots \ldots \ldots \ldots \ldots \ldots \ldots \ldots \\
\text { Renter or other } \ldots \ldots \ldots \ldots \ldots \ldots \ldots\end{array}$ & $\begin{array}{l}93.8 \\
72.3\end{array}$ & $\begin{array}{c}100.0 \\
*\end{array}$ & $\begin{array}{r}17.5 \\
5.6\end{array}$ & $\begin{array}{r}10.8 \\
2.1\end{array}$ & $\begin{array}{r}15.4 \\
4.5\end{array}$ & $\begin{array}{l}8.0 \\
5.3\end{array}$ & $\begin{array}{r}100.0 \\
74.5\end{array}$ & $\begin{array}{r}100.0 \\
92.8\end{array}$ \\
\hline $\begin{array}{l}\text { Percentile of net worth } \\
\text { Less than } 25 \ldots \ldots \ldots \ldots \\
25-49.9 \ldots \ldots \ldots \ldots \ldots \\
50-74.9 \ldots \ldots \ldots \ldots \ldots \\
75-89.9 \ldots \ldots \ldots \ldots \ldots \\
90-100\end{array}$ & $\begin{array}{l}69.5 \\
91.2 \\
93.3 \\
94.5 \\
93.6\end{array}$ & $\begin{array}{l}13.8 \\
72.1 \\
92.8 \\
95.3 \\
96.9\end{array}$ & $\begin{array}{r}1.2 \\
7.1 \\
11.9 \\
26.2 \\
47.7\end{array}$ & $\begin{array}{c}* \\
3.7 \\
7.7 \\
16.4 \\
27.3\end{array}$ & $\begin{array}{r}1.3 \\
6.2 \\
11.6 \\
17.9 \\
45.1\end{array}$ & $\begin{array}{r}2.4 \\
6.5 \\
7.8 \\
7.5 \\
18.5\end{array}$ & $\begin{array}{l}71.6 \\
97.7 \\
99.5 \\
99.0 \\
99.6\end{array}$ & $\begin{array}{r}90.9 \\
100.0 \\
100.0 \\
100.0 \\
100.0\end{array}$ \\
\hline
\end{tabular}


9. Family holdings of nonfinancial assets and of any asset, by selected characteristics of families and type of asset, 2004 and 2007 surveys-Continued

B. 2007 Survey of Consumer Finances-continued

\begin{tabular}{|c|c|c|c|c|c|c|c|c|}
\hline Family characteristic & Vehicles & $\begin{array}{l}\text { Primary } \\
\text { residence }\end{array}$ & $\begin{array}{l}\text { Other } \\
\text { residential } \\
\text { property }\end{array}$ & $\begin{array}{c}\text { Equity in } \\
\text { nonresidential } \\
\text { property }\end{array}$ & $\begin{array}{c}\text { Business } \\
\text { equity }\end{array}$ & Other & $\begin{array}{c}\text { Any } \\
\text { nonfinancial } \\
\text { asset }\end{array}$ & Any asset \\
\hline & \multicolumn{8}{|c|}{ Median value of holdings for families holding asset (thousands of 2007 dollars) } \\
\hline All families & 15.5 & 200.0 & 146.0 & 75.0 & 100.5 & 14.0 & 177.4 & 221.5 \\
\hline 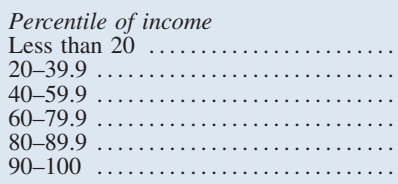 & $\begin{array}{r}5.6 \\
9.2 \\
14.6 \\
20.4 \\
25.4 \\
33.9\end{array}$ & $\begin{array}{l}100.0 \\
120.0 \\
150.0 \\
215.0 \\
300.0 \\
500.0\end{array}$ & $\begin{array}{r}60.0 \\
57.5 \\
100.0 \\
120.0 \\
175.0 \\
324.0\end{array}$ & $\begin{array}{r}65.0 \\
60.0 \\
40.0 \\
71.0 \\
72.0 \\
175.0\end{array}$ & $\begin{array}{r}100.0 \\
25.0 \\
53.7 \\
81.0 \\
100.0 \\
500.0\end{array}$ & $\begin{array}{r}3.0 \\
6.0 \\
10.0 \\
15.0 \\
20.0 \\
75.0\end{array}$ & $\begin{array}{r}40.0 \\
77.2 \\
139.0 \\
246.3 \\
360.1 \\
799.9\end{array}$ & $\begin{array}{r}23.5 \\
84.9 \\
183.5 \\
342.8 \\
558.1 \\
1,358.4\end{array}$ \\
\hline 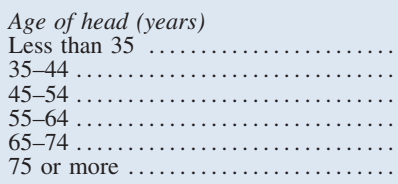 & $\begin{array}{r}13.3 \\
17.4 \\
18.7 \\
17.4 \\
14.6 \\
9.4\end{array}$ & $\begin{array}{l}175.0 \\
205.0 \\
230.0 \\
210.0 \\
200.0 \\
150.0\end{array}$ & $\begin{array}{r}85.0 \\
150.0 \\
150.0 \\
157.0 \\
150.0 \\
100.0\end{array}$ & $\begin{array}{r}50.0 \\
50.0 \\
80.0 \\
90.0 \\
75.0 \\
110.0\end{array}$ & $\begin{array}{r}59.9 \\
86.0 \\
100.0 \\
116.3 \\
415.0 \\
250.0\end{array}$ & $\begin{array}{r}8.0 \\
10.0 \\
15.0 \\
20.0 \\
20.0 \\
25.0\end{array}$ & $\begin{array}{r}30.9 \\
182.6 \\
224.9 \\
233.1 \\
212.2 \\
157.1\end{array}$ & $\begin{array}{r}38.8 \\
222.3 \\
306.0 \\
347.0 \\
303.3 \\
219.3\end{array}$ \\
\hline $\begin{array}{l}\text { Family structure } \\
\text { Single with child(ren) } \ldots \ldots \ldots \ldots \ldots \\
\text { Single, no child, age less than } 55 \ldots \ldots \\
\text { Single, no child, age } 55 \text { or more } \ldots \ldots \\
\text { Couple with child(ren) } \ldots \ldots \ldots \ldots \ldots \ldots \\
\text { Couple, no child } \ldots \ldots \ldots \ldots \ldots \ldots \ldots \ldots\end{array}$ & $\begin{array}{r}8.3 \\
9.8 \\
7.4 \\
20.8 \\
20.6\end{array}$ & $\begin{array}{l}165.0 \\
155.0 \\
140.0 \\
225.0 \\
230.0\end{array}$ & $\begin{array}{r}90.0 \\
120.0 \\
80.0 \\
133.0 \\
165.0\end{array}$ & $\begin{array}{l}71.0 \\
48.8 \\
75.0 \\
50.0 \\
85.0\end{array}$ & $\begin{array}{r}100.0 \\
50.0 \\
300.0 \\
81.8 \\
130.0\end{array}$ & $\begin{array}{r}9.0 \\
9.0 \\
10.0 \\
12.5 \\
20.0\end{array}$ & $\begin{array}{r}106.9 \\
52.0 \\
133.0 \\
218.0 \\
235.6\end{array}$ & $\begin{array}{r}116.4 \\
52.6 \\
177.1 \\
292.8 \\
312.1\end{array}$ \\
\hline $\begin{array}{l}\text { Education of head } \\
\text { No high school diploma } \ldots \ldots \ldots \ldots \ldots \\
\text { High school diploma } \ldots \ldots \ldots \ldots \ldots \ldots \\
\text { Some college } \ldots \ldots \ldots \ldots \ldots \ldots \ldots \ldots \\
\text { College degree } \ldots \ldots \ldots \ldots \ldots \ldots \ldots \ldots\end{array}$ & $\begin{array}{l}10.4 \\
13.3 \\
14.6 \\
19.9\end{array}$ & $\begin{array}{l}122.5 \\
150.0 \\
192.0 \\
280.0\end{array}$ & $\begin{array}{r}65.0 \\
76.0 \\
100.0 \\
200.0\end{array}$ & $\begin{array}{r}125.0 \\
50.0 \\
52.8 \\
90.0\end{array}$ & $\begin{array}{r}66.0 \\
100.0 \\
81.2 \\
125.4\end{array}$ & $\begin{array}{r}13.2 \\
7.3 \\
13.0 \\
20.0\end{array}$ & $\begin{array}{r}84.4 \\
137.7 \\
157.3 \\
289.4\end{array}$ & $\begin{array}{r}64.6 \\
161.8 \\
186.3 \\
435.4\end{array}$ \\
\hline $\begin{array}{l}\text { Race or ethnicity of respondent } \\
\text { White non-Hispanic } \ldots \ldots \ldots \ldots \ldots \ldots \\
\text { Nonwhite or Hispanic } \ldots \ldots \ldots \ldots \ldots \ldots\end{array}$ & $\begin{array}{l}17.1 \\
12.0\end{array}$ & $\begin{array}{l}200.0 \\
180.0\end{array}$ & $\begin{array}{l}136.5 \\
175.0\end{array}$ & $\begin{array}{l}75.0 \\
62.7\end{array}$ & $\begin{array}{r}112.5 \\
60.0\end{array}$ & $\begin{array}{r}15.0 \\
8.0\end{array}$ & $\begin{array}{l}203.8 \\
102.0\end{array}$ & $\begin{array}{r}271.0 \\
89.2\end{array}$ \\
\hline $\begin{array}{l}\text { Current work status of head } \\
\text { Working for someone else } \ldots \ldots \ldots \ldots \\
\text { Self-employed } \ldots \ldots \ldots \ldots \ldots \ldots \ldots \ldots \\
\text { Retired } \ldots \ldots \ldots \ldots \ldots \ldots \ldots \ldots \ldots \\
\text { Other not working } \ldots \ldots \ldots \ldots \ldots \ldots \ldots\end{array}$ & $\begin{array}{r}17.0 \\
22.1 \\
11.4 \\
6.9\end{array}$ & $\begin{array}{l}200.0 \\
300.0 \\
155.0 \\
160.0\end{array}$ & $\begin{array}{l}120.0 \\
293.0 \\
100.0 \\
130.5\end{array}$ & $\begin{array}{r}52.8 \\
152.5 \\
75.0 \\
48.8\end{array}$ & $\begin{array}{r}50.0 \\
150.0 \\
212.6 \\
103.1\end{array}$ & $\begin{array}{r}10.0 \\
50.0 \\
13.2 \\
2.5\end{array}$ & $\begin{array}{r}167.1 \\
455.0 \\
156.0 \\
29.3\end{array}$ & $\begin{array}{r}213.3 \\
543.9 \\
203.5 \\
28.9\end{array}$ \\
\hline $\begin{array}{l}\text { Current occupation of head } \\
\text { Managerial or professional } \ldots \ldots \ldots \ldots \\
\text { Technical, sales, or services } \ldots \ldots \ldots \ldots \\
\text { Other occupation } \ldots \ldots \ldots \ldots \ldots \ldots \\
\text { Retired or other not working } \ldots \ldots \ldots \ldots\end{array}$ & $\begin{array}{l}20.2 \\
14.4 \\
16.7 \\
10.4\end{array}$ & $\begin{array}{l}270.0 \\
200.0 \\
157.9 \\
155.0\end{array}$ & $\begin{array}{r}200.0 \\
125.0 \\
90.0 \\
100.0\end{array}$ & $\begin{array}{r}105.0 \\
85.0 \\
37.0 \\
75.0\end{array}$ & $\begin{array}{r}200.0 \\
40.0 \\
68.6 \\
196.9\end{array}$ & $\begin{array}{l}20.0 \\
15.0 \\
10.8 \\
12.5\end{array}$ & $\begin{array}{l}278.9 \\
155.0 \\
135.6 \\
146.7\end{array}$ & $\begin{array}{l}411.2 \\
187.0 \\
157.6 \\
177.1\end{array}$ \\
\hline 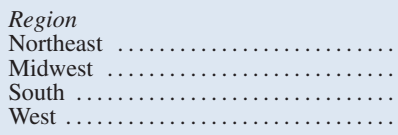 & $\begin{array}{l}14.5 \\
14.6 \\
15.6 \\
17.1\end{array}$ & $\begin{array}{l}275.0 \\
155.0 \\
160.0 \\
300.0\end{array}$ & $\begin{array}{l}190.0 \\
110.0 \\
120.0 \\
210.0\end{array}$ & $\begin{array}{r}112.0 \\
52.8 \\
71.5 \\
90.0\end{array}$ & $\begin{array}{r}150.0 \\
112.4 \\
93.8 \\
101.4\end{array}$ & $\begin{array}{l}20.0 \\
10.0 \\
15.0 \\
14.0\end{array}$ & $\begin{array}{l}250.0 \\
157.5 \\
145.8 \\
251.6\end{array}$ & $\begin{array}{l}290.4 \\
204.7 \\
180.9 \\
293.2\end{array}$ \\
\hline $\begin{array}{l}\text { Urbanicity } \\
\text { Metropolitan statistical area (MSA) } \ldots \\
\text { Non-MSA ...................... }\end{array}$ & $\begin{array}{l}15.8 \\
14.5\end{array}$ & $\begin{array}{l}220.0 \\
115.0\end{array}$ & $\begin{array}{r}150.0 \\
80.0\end{array}$ & $\begin{array}{l}82.5 \\
50.0\end{array}$ & $\begin{array}{l}105.0 \\
100.0\end{array}$ & $\begin{array}{l}13.5 \\
22.0\end{array}$ & $\begin{array}{l}194.0 \\
118.6\end{array}$ & $\begin{array}{l}243.9 \\
149.2\end{array}$ \\
\hline $\begin{array}{l}\text { Housing status } \\
\text { Owner } \ldots \ldots \ldots \ldots \ldots \ldots \ldots \ldots \ldots \ldots \ldots \ldots \ldots \ldots \ldots \\
\text { Renter or other } \ldots \ldots \ldots \ldots \ldots \ldots \ldots\end{array}$ & $\begin{array}{r}18.4 \\
8.6\end{array}$ & $\begin{array}{c}200.0 \\
*\end{array}$ & $\begin{array}{r}150.0 \\
85.0\end{array}$ & $\begin{array}{l}80.0 \\
38.0\end{array}$ & $\begin{array}{r}113.4 \\
50.0\end{array}$ & $\begin{array}{r}20.0 \\
5.4\end{array}$ & $\begin{array}{r}253.5 \\
10.1\end{array}$ & $\begin{array}{r}344.2 \\
13.6\end{array}$ \\
\hline 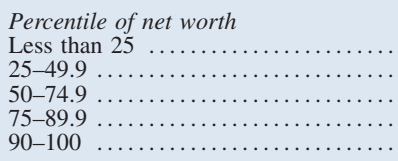 & $\begin{array}{r}6.9 \\
13.1 \\
17.5 \\
22.0 \\
31.1\end{array}$ & $\begin{array}{r}81.0 \\
100.0 \\
200.0 \\
317.2 \\
550.0\end{array}$ & $\begin{array}{r}12.0 \\
30.0 \\
60.0 \\
146.0 \\
400.0\end{array}$ & $\begin{array}{r}* \\
25.0 \\
38.4 \\
82.5 \\
266.7\end{array}$ & $\begin{array}{r}4.0 \\
20.0 \\
67.6 \\
125.0 \\
690.0\end{array}$ & $\begin{array}{r}1.3 \\
7.5 \\
13.0 \\
30.0 \\
75.0\end{array}$ & $\begin{array}{r}8.6 \\
95.8 \\
229.1 \\
443.7 \\
1,160.0\end{array}$ & $\begin{array}{r}8.1 \\
107.8 \\
304.3 \\
687.1 \\
2,104.0\end{array}$ \\
\hline $\begin{array}{l}\text { MEMo } \\
\text { Mean value of holdings for } \\
\text { families holding asset } \ldots\end{array}$ & 22.0 & 302.4 & 335.6 & 309.4 & 1071.1 & 80.7 & 469.5 & 668.5 \\
\hline
\end{tabular}

Note: See note to table 8.

* Ten or fewer observations. 
creased for families headed by a person who was retired, self-employed, or otherwise not working; for single families without children; and for families living in the Northeast or the West.

The median market value of vehicles for those who owned at least one vehicle declined 0.6 percent from 2004 to 2007, and the mean declined 0.5 percent. $^{35}$ The median value of vehicle holdings fell most substantially for families in the other-not-working work-status group, families in the Northeast, and the 55-to-64 age group. Other relatively large declines in the median included those for the highest three income and wealth groups. For most other families, the median rose or held about steady. These trends are essentially the opposite of those observed between 2001 and 2004, when median values fell for the lowest two income and wealth groups, the two oldest and the youngest age groups, nonwhite or Hispanic families, renters, and families headed by a person who was retired. However, continuing a trend, the share of the total value of owned vehicles attributable to sport utility vehicles rose over the recent period, from 19.1 percent to 20.9 percent (data not shown in the tables).

Some families have vehicles that they lease or that are provided to them by an employer for personal use. The share of families having a vehicle from any source rose 0.3 percentage point over the recent period, to 89.6 percent. The small difference between this rate and the ownership rate for personally owned vehicles belies a larger change in the rates of holding for leased and employer-provided vehicles. The proportion of families with a leased vehicle rose, from 4.0 percent to 5.2 percent, while that of families with an employer-provided vehicle fell, from 7.7 percent to 6.8 percent.

\section{Primary Residence and Other Residential Real Estate}

The homeownership rate turned down slightly over the $2004-07$ period, falling 0.5 percentage point, to 68.6 percent. ${ }^{36}$ In 2007, groups that had an ownership rate less than the overall rate included nonwhite or

35. Survey respondents are asked to provide the year, make, and model of each of their cars, vans, sport utility vehicles, and trucks. This information is used to obtain market prices from data collected by the National Automobile Dealers Association and a variety of other sources. For other types of vehicles, the respondent is asked to provide a best estimate of the current value.

36. This measure of primary residences comprises mobile homes and their sites, the parts of farms and ranches not used for a farming or ranching business, condominiums, cooperatives, townhouses, other single-family homes, and other permanent dwellings. The 2004 and 2007 SCF estimates of homeownership differ only marginally from those of the CPS for a comparable specification of household; the CPS shows an identical decline in the homeownership rate.
Hispanic families, families with relatively low income or wealth, families living in the Northeast or the West, single families, and families headed by a person who was neither working nor retired, who was aged less than 45 , or who had less than a high school diploma or only some college education. Over the three-year period, homeownership rose most for the lowest quintile of the income distribution; families headed by a person aged 65 to 74; families headed by a person who was self-employed or working in a technical, sales, or service job; or families headed by a high school graduate. The largest declines in the homeownership rate were for single families with children and families in the 75-or-more age group or the other-not-working work-status group.

Housing wealth represents a large component of total family wealth; in 2007 , the primary residence accounted for 31.8 percent of total family assets. Over the 2004-07 period, this percentage declined slightly overall. The relative importance of housing in the total asset portfolio varies substantially over the income distribution, with housing generally constituting a smaller share of the portfolio with increasing levels of income (table 9.1).

9.1.

\begin{tabular}{|c|c|c|}
\hline \multirow{2}{*}{ Family characteristic } & \multicolumn{2}{|c|}{$\begin{array}{l}\text { House value as a percentage } \\
\text { of all assets of group }\end{array}$} \\
\hline & $\begin{array}{c}2007 \\
\text { (percent) }\end{array}$ & $\begin{array}{c}\text { Change, 2004-07 } \\
\text { (percentage points) }\end{array}$ \\
\hline All families . & 31.8 & -0.5 \\
\hline $\begin{array}{l}\text { Percentile of income } \\
\text { Less than } 20 \ldots \ldots \ldots \ldots \\
20-39.9 \ldots \ldots \ldots \ldots \ldots \ldots \\
40-59.9 \ldots \ldots \ldots \ldots \ldots \ldots \\
60-79.9 \ldots \ldots \ldots \ldots \ldots \ldots \\
80-89.9 \ldots \ldots \ldots \ldots \ldots \ldots \\
90-100 \quad \ldots \ldots \ldots \ldots \ldots \ldots\end{array}$ & $\begin{array}{l}47.1 \\
51.8 \\
48.4 \\
45.3 \\
44.5 \\
19.8\end{array}$ & $\begin{array}{r}-1.5 \\
2.2 \\
-1.5 \\
2.5 \\
2.7 \\
-1.1\end{array}$ \\
\hline
\end{tabular}

The median and mean values of the primary residences of homeowners rose from 2004 to 2007; overall, the median increased 13.8 percent, and the mean rose 11.5 percent. These percentage gains in the median and mean translated into large dollar gains: $\$ 24,300$ for the median and $\$ 31,300$ for the mean. Homeowners in all demographic groups saw gains in the median, most of them substantial. The only breaks in the pattern of gains in median values across groups were a decline of 4.4 percent for families headed by a person aged 55 to 64 and a decline of 2.7 percent for homeowners in the Midwest. One of the largest increases was the 26.1 percent rise in the median value of primary residences for nonwhite or Hispanic families; in contrast, the median for other families rose 10.4 percent. Other sizable increases included those for families headed by a person without a high 
school diploma (48.7 percent) and for families in the bottom income quintile (30.0 percent).

As discussed earlier, the national housing market continued to decline after data collection for the 2007 SCF had been completed. Assuming that homeownership did not change and that changes in house prices occurred uniformly across all homeowners in a given state, then the state-level purchase-only LoanPerformance Home Price Index can be used to approximate the effects of declines in house prices from the time of the interview until October 2008. Under these assumptions, the median value falls from $\$ 200,000$ for 2007 to $\$ 181,600$, still a gain of 3.4 percent from 2004; the mean falls from $\$ 302,400$ for 2007 to $\$ 265,600$ in October 2008, a decline of 2.0 percent from 2004.

In 2007, 13.7 percent of families owned some form of residential real estate other than a primary residence (second homes, time-shares, one- to fourfamily rental properties, and other types of residential properties), a level that is up 1.2 percentage points from the figure in 2004. ${ }^{37}$ Although the survey does not ask directly about ownership of second homes, such homes should largely be captured as residential properties that are owned 100 percent by the family and for which no rent was collected; in 2007, 6.1 percent of families had at least one such property, up 1.5 percentage points from 2004 (data not shown in the tables).

Ownership of other residential real estate is much more common among the highest income and wealth groups, the age groups between 45 and 74 , and families headed by a self-employed person, a person working in a managerial or professional occupation, or a person who was a college graduate. The median and mean values of other residential real estate increased proportionately more than the median and mean values of primary residences over the recent period; the median rose 33.0 percent, and the mean rose 14.3 percent. Most of the demographic groups saw substantial gains in the median. Declines in median values were observed for several groups, including the youngest and oldest age groups, families whose head had not attended college, and families headed by a person who was retired.

\section{Net Equity in Nonresidential Real Estate}

The ownership of nonresidential real estate fell slightly, to 8.1 percent of families in $2007 .{ }^{38}$ Owner-

37. This measure of residential real estate also includes outstanding balances on loans that the family may have made to finance the sale of properties they previously owned. ship follows approximately the same relative distribution across demographic groups as does the ownership of other residential real estate. Changes in ownership during the recent period were mixed across demographic groups. Ownership increased modestly in the top two deciles of the income distribution, while it decreased modestly in most of the lower portion of the distribution. By educational attainment, ownership increased only among families headed by a person with a high school diploma. Overall, the median value of such property for owners rose 13.8 percent, and the mean fell 5.5 percent. Particularly large gains in the median value were seen for families in the lowest income group, single-parent families, and families headed by a person without a high school diploma-all groups with below-average ownership rates.

\section{Net Equity in Privately Held Businesses}

The share of families that owned a privately held business interest edged up 0.5 percentage point during the recent period, to 12.0 percent. ${ }^{39}$ The propor-

38. Nonresidential real estate comprises the following types of properties unless they are owned through a business: commercial property, rental property with five or more units, farm and ranch land, undeveloped land, and all other types of nonresidential real estate. Most often, nonresidential real estate properties are functionally more like a business than a residential property. They may have a number of owners, they are typically worth a considerable amount, and they often carry large mortgages, which appear to be paid from the revenues from the property, not the family's other income. As in the case of privately owned businesses, the value of the property in this analysis is taken to be the net value.

39. The forms of business in this category are sole proprietorships, limited partnerships, other types of partnerships, subchapter S corporations and other types of corporations that are not publicly traded, limited liability companies, and other types of private businesses. If the family surveyed lived on a farm or ranch that was used at least in part for agricultural business, the value of that part, net of the corresponding share of associated debts, is included with other business assets.

In the survey, self-employment status and business ownership are independently determined. Among the 12.0 percent of families with a business in 2007, 70.1 percent had a family head or the spouse or partner of the head who was self-employed; among the 12.5 percent of families in which either the head or the spouse or partner of the head was self-employed, 67.5 percent owned a business (data not shown in the tables).

The 2004 and 2007 surveys differ in the ways that business ownership was determined. In both surveys, respondents were asked directly about business ownership. In the 2004 and earlier surveys, it had been noticed at the stage of data editing that some respondents had reported themselves as self-employed and as having substantial associated business assets but had failed to report ownership of a business, perhaps as a result of some confusion about the intent of the business ownership question; where possible, the data were corrected for such misunderstandings. Beginning with the 2007 survey, a new follow-up question was asked of every person who was reported as being self-employed but who had not been noted as working for a business owned by the family. The question asked whether a business with some value was associated with the self-employment. If so, then several additional questions were asked about the business's value and 
tion has changed little over the past several surveys. Ownership of this type of asset tends to increase with income, wealth, and education and to be the highest for families headed by a person who is aged 35 to 64 , who is married or living with a partner, or who has at least some college education. Business ownership is about three times as prevalent among homeowners as renters; it is generally lowest in the Northeast and highest in the West. Over the recent three-year period, increases in ownership were largely concentrated in the highest income and net worth deciles. By region, ownership declined in the Northeast, while increases were reported in the South and West. Breaking a pattern seen in the preceding three years, ownership also increased substantially among families headed by a person who was self-employed.

As noted earlier, equity in privately held businesses makes up a large portion of families' total nonfinancial assets. This pattern has strengthened over the recent period. Across the income distribution, the share of assets attributable to business equity has a U-shape, with the largest shares at the top and bottom of the income distribution (table 9.2).

9.2 .

\begin{tabular}{|c|c|c|}
\hline \multirow{2}{*}{ Family characteristic } & \multicolumn{2}{|c|}{$\begin{array}{l}\text { Net equity in business as a } \\
\text { percentage of all assets }\end{array}$} \\
\hline & $\begin{array}{c}2007 \\
\text { (percent) }\end{array}$ & $\begin{array}{l}\text { Change, 2004-07 } \\
\text { (percentage points) }\end{array}$ \\
\hline All families . & 19.6 & 3.0 \\
\hline $\begin{array}{l}\text { Percentile of income } \\
\text { Less than } 20 \ldots \ldots \ldots \ldots \\
20-39.9 \ldots \ldots \ldots \ldots \ldots \ldots \\
40-59.9 \ldots \ldots \ldots \ldots \ldots \ldots \\
60-79.9 \ldots \ldots \ldots \ldots \ldots \ldots \\
80-89.9 \ldots \ldots \ldots \ldots \ldots \ldots \\
90-100 \ldots \ldots \ldots \ldots \ldots \ldots\end{array}$ & $\begin{array}{r}18.8 \\
4.2 \\
9.1 \\
6.8 \\
11.4 \\
28.1\end{array}$ & $\begin{array}{r}4.2 \\
-5.0 \\
3.2 \\
-1.4 \\
4.7 \\
3.7\end{array}$ \\
\hline
\end{tabular}

The median holding of business equity for those having any such equity declined 8.5 percent while the mean increased 27.4 percent. These changes follow a decline of 6.2 percent in the median and an increase of 11.4 percent in the mean between the 2001 and 2004 surveys. In 2007, median values were generally increasing in income, age, and net worth. Median net equity in businesses owned by white non-Hispanic families and homeowners are substantially higher than for the complementary groups. Over the recent three-year period, large increases in median net equity in businesses were observed in the lowest income quintile, in the oldest two age categories, in single families headed by a person aged 55 or older, and in

income, and that information was introduced into the appropriate places in the section of the survey covering businesses. It is possible that the systematic approach in 2007 discovered more private businesses than had previously been detected through editing. families in the other-not-working work-status group. Changes in the medians for other categories included increases and decreases of smaller magnitudes.

The SCF classifies privately owned business interests into those in which the family has an active management role and those in which it does not. Of families having any business interests in 2007, 92.0 percent had an active role, and 12.0 percent had a non-active role; 3.9 percent had interests of both types (data not shown in the tables). In terms of assets, actively managed interests accounted for 89.1 percent of total privately owned business interests. The median number of actively managed businesses was 1 . The businesses reported in the survey were a mixture of very small businesses with moderate values and businesses with substantially greater values.

The SCF attempts to collect information about items owned or owed by a family's business interests separately from items owned or owed directly by the family. But, in practice, the balance sheet of a business that is actively managed by a family is not always separate from that of the family itself. Families often use personal assets as collateral or guarantees for loans for the businesses, or they loan personal funds to their businesses. In 2007, 17.8 percent of families with actively managed businesses reported using personal assets as collateral, and 17.5 percent of families reported lending the business money; both percentages are down from their 2004 levels of 19.7 percent and 20.2 percent, respectively (data not shown in the tables).

Families with more than one actively managed business are asked to report which business is most important; that business is designated as the primary one. ${ }^{40}$ In 2007 , the vast majority of primary businesses operated in an industry other than manufacturing; the most common organizational form of those businesses was sole proprietorship, and the median number of employees was 2. However, primary actively managed businesses with more than two employees accounted for 80.4 percent of the value of all such businesses, and the largest shares of value were attributable to businesses organized as subchapter S corporations or limited liability companies, each of which accounted for just more than 30 percent. These patterns are also typical of those observed in the earlier surveys.

40. For families with only one business, that business is, by default, considered the primary one. In 2007, primary actively managed businesses accounted for 78.0 percent of the value of all actively managed businesses. 
10. Family holdings of unrealized capital gains on selected assets as a share of total assets, by selected characteristics of families, 1998-2007 surveys

Percent

\begin{tabular}{|c|c|c|c|c|c|c|c|c|c|c|c|c|c|c|c|c|}
\hline \multirow[b]{2}{*}{ Family characteristic } & \multicolumn{4}{|c|}{1998} & \multicolumn{4}{|c|}{2001} & \multicolumn{4}{|c|}{2004} & \multicolumn{4}{|c|}{2007} \\
\hline & $\begin{array}{l}\text { Real } \\
\text { estate }\end{array}$ & $\begin{array}{l}\text { Busi- } \\
\text { ness }\end{array}$ & $\begin{array}{c}\text { Finan- } \\
\text { cial }\end{array}$ & All & $\begin{array}{l}\text { Real } \\
\text { estate }\end{array}$ & $\begin{array}{c}\text { Busi- } \\
\text { ness }\end{array}$ & $\begin{array}{c}\text { Finan- } \\
\text { cial }\end{array}$ & All & $\begin{array}{l}\text { Real } \\
\text { estate }\end{array}$ & $\begin{array}{c}\text { Busi- } \\
\text { ness }\end{array}$ & $\begin{array}{c}\text { Finan- } \\
\text { cial }\end{array}$ & All & $\begin{array}{l}\text { Real } \\
\text { estate }\end{array}$ & $\begin{array}{l}\text { Busi- } \\
\text { ness }\end{array}$ & $\begin{array}{c}\text { Finan- } \\
\text { cial }\end{array}$ & All \\
\hline All families & 13.5 & 11.6 & 4.3 & 29.3 & 14.8 & 11.6 & 2.3 & 28.7 & 18.7 & 10.9 & 1.1 & 30.7 & 18.9 & 14.2 & 2.6 & 35.8 \\
\hline Percentile of income & & & & & & & & & & & & & & & & \\
\hline Less than $20 \ldots \ldots$. & 27.6 & 4.9 & .3 & 32.8 & 26.7 & 2.0 & -.1 & 28.6 & 29.3 & 7.7 & -.6 & 36.4 & 30.5 & 10.6 & 1.4 & 42.5 \\
\hline $20-39.9 \ldots \ldots$ & 22.5 & 2.3 & 1.3 & 26.1 & 27.0 & 3.9 & -.3 & 30.7 & 28.3 & 5.9 & .3 & 34.5 & 31.4 & 3.2 & .3 & 35.0 \\
\hline $40-59.9$ & 20.8 & 5.6 & 1.3 & 27.7 & 18.8 & 3.9 & .2 & 22.9 & 25.9 & 3.0 & .5 & 29.4 & 24.7 & 5.6 & .8 & 31.1 \\
\hline $60-79.9$ & 16.0 & 6.3 & 2.4 & 24.6 & 17.0 & 5.2 & 1.7 & 24.0 & 23.1 & 4.0 & .5 & 27.6 & 23.1 & 3.8 & 1.6 & 28.6 \\
\hline $80-89.9$ & 14.1 & 6.5 & 2.8 & 23.4 & 15.7 & 7.8 & 1.8 & 25.3 & 19.4 & 4.4 & .8 & 24.7 & 23.8 & 8.8 & .9 & 33.6 \\
\hline $90-100 \quad \ldots \ldots \ldots \ldots \ldots$ & 9.1 & 17.2 & 6.4 & 32.7 & 11.4 & 16.9 & 3.3 & 31.6 & 14.3 & 16.6 & 1.6 & 32.5 & 13.8 & 20.8 & 3.9 & 38.5 \\
\hline Age of head (years) & & & & & & & & & & & & & & & & \\
\hline Less than $35 \ldots . .$. & 7.1 & 7.4 & .9 & 15.3 & 8.1 & 10.7 & 2.1 & 20.8 & 13.4 & 7.5 & -.4 & 20.4 & 12.6 & 14.6 & 1.0 & 28.2 \\
\hline $35-44 \ldots \ldots \ldots$ & 9.4 & 11.7 & 3.1 & 24.2 & 12.7 & 14.8 & .2 & 27.7 & 16.2 & 12.0 & 1.4 & 29.6 & 16.2 & 12.3 & .4 & 28.9 \\
\hline $45-54 \ldots$ & 10.8 & 15.7 & 2.8 & 29.3 & 12.9 & 12.6 & 2.0 & 27.5 & 16.7 & 13.5 & 1.1 & 31.3 & 18.3 & 15.5 & 2.1 & 36.0 \\
\hline $55-64$ & 12.9 & 12.9 & 6.1 & 32.0 & 13.8 & 12.5 & 2.0 & 28.3 & 19.0 & 11.8 & $\dagger$ & 30.8 & 17.4 & 15.4 & 3.2 & 36.0 \\
\hline $65-74 \ldots \ldots$ & 18.3 & 9.1 & 6.0 & 33.5 & 20.0 & 10.3 & 3.5 & 33.8 & 20.8 & 8.8 & 2.1 & 31.8 & 20.6 & 13.8 & 4.0 & 38.4 \\
\hline 75 or more & 25.5 & 5.0 & 5.3 & 35.8 & 21.1 & 5.1 & 5.2 & 31.4 & 26.5 & 5.5 & 2.4 & 34.4 & 28.4 & 11.0 & 4.0 & 43.5 \\
\hline МЕмо & & & & & & & & & & & & & & & & \\
\hline $\begin{array}{l}\text { Percent of families with } \\
\text { any such gains } \ldots . . . .\end{array}$ & 65.5 & 10.7 & 26.3 & 71.0 & 67.2 & 11.6 & 27.6 & 72.1 & 68.8 & 11.1 & 25.1 & 73.0 & 69.0 & 11.5 & 21.7 & 72.4 \\
\hline $\begin{array}{l}\text { Median for those with } \\
\text { any such gains } \ldots \ldots \text {. }\end{array}$ & 37.8 & 39.5 & 4.6 & 39.5 & 45.1 & 59.6 & .6 & 46.8 & 61.0 & 49.4 & .7 & 59.3 & 71.0 & 50.0 & 3.5 & 75.0 \\
\hline $\begin{array}{l}\text { Mean for those with } \\
\text { any such gains .... }\end{array}$ & 86.2 & 453.7 & 67.8 & 172.9 & 116.6 & 530.4 & 43.9 & 210.4 & 157.6 & 567.6 & 24.3 & 243.1 & 179.2 & 805.1 & 79.3 & 322.9 \\
\hline
\end{tabular}

Note: See note to table 1 .

$\dagger$ Less than 0.05 (\$50).

\section{Other Nonfinancial Assets}

In 2007, ownership of the remaining nonfinancial assets (tangible items including artwork, jewelry, precious metals, antiques, hobby equipment, and collectibles) was not very widespread and decreased marginally compared with the level in the previous survey period, to 7.2 percent. Among other nonfinancial assets, the most commonly held items are antiques and other collectibles, which were held by only 3.6 percent of families. The composition of other nonfinancial assets changed little from 2004 (table 9.3).

9.3.

\begin{tabular}{c|c|c}
\hline \multirow{2}{*}{$\begin{array}{c}\text { Type of other } \\
\text { nonfinancial asset }\end{array}$} & \multicolumn{2}{|c}{ All families } \\
\cline { 2 - 3 } & $\begin{array}{c}2007 \\
\text { (percent) }\end{array}$ & $\begin{array}{c}\text { Change, 2004-07 } \\
\text { (percentage points) }\end{array}$ \\
\hline Gold, silver, or jewelry ..... & 2.1 & $\dagger$ \\
Antiques, collectibles ........ & 3.6 & -.2 \\
Art objects ................ & 1.8 & -.2 \\
Other ................... & .9 & -.3 \\
\hline
\end{tabular}

$\dagger$ Less than 0.05 percent.

Groups most likely to hold other nonfinancial assets generally include families in the top two deciles of the income distribution, families headed by a college graduate, homeowners, and families in the top two quartiles of the net worth distribution. Minor changes in holdings were evident across all the demographic groups. For families having such assets, the median value fell 15.2 percent over the recent period, and the mean rose 16.7 percent. Across income groups, median holdings rose for families in the top three groups and declined for families in the second and third quintiles.

\section{Unrealized Capital Gains}

Changes in the values of assets such as stock, real estate, and businesses are a key determinant of changes in families' net worth. Unrealized gains are increases in the value of assets that are yet to be sold. To obtain information on this part of net worth, the survey asks about changes in value from the time of purchase for certain key assets-publicly traded stocks, pooled investment funds, the primary residence, other real estate, and the current tax basis of businesses. ${ }^{41}$ Among families with any unrealized capital gain, the median value of that gain moved up 26.5 percent over the 2004-07 period, and the mean moved up 32.8 percent (table 10). These unrealized capital gains are a very important part of family assets; in 2007, they represented 35.8 percent of total family assets, a fraction larger than that observed in any other SCF since 1989. Unrealized capital gains

41. The survey does not collect information on capital gains on every asset for which such gains are possible. Most important, it does not collect such information for retirement accounts. 
11. Amount of debt of all families, distributed by type of debt, 1998-2007 surveys

Percent

\begin{tabular}{|c|c|c|c|c|}
\hline Type of debt & 1998 & 2001 & 2004 & 2007 \\
\hline Secured by residential property & & & & \\
\hline Primary residence...$\ldots \ldots$. & 71.4 & 75.2 & 75.2 & 74.7 \\
\hline Other ................ & 7.5 & 6.2 & 8.5 & 10.1 \\
\hline Lines of credit not secured & & & & \\
\hline by residential property & .3 & .5 & .7 & .4 \\
\hline Installment loans $\ldots \ldots \ldots \ldots$ & 13.1 & 12.3 & 11.0 & 10.2 \\
\hline Credit card balances ......... & 3.9 & 3.4 & 3.0 & 3.5 \\
\hline Other $\ldots \ldots \ldots \ldots \ldots \ldots$ & 3.7 & 2.3 & 1.6 & 1.1 \\
\hline Total & 100 & 100 & 100 & 100 \\
\hline
\end{tabular}

NotE: See note to table 1.

tend to increase with age as a fraction of total family assets. The fraction of total family assets attributable to unrealized capital gains decreases and then increases across income groups. In 2007, this fraction was lowest for families in the third income quintile. The largest component of unrealized capital gains in all years of the SCF shown was real estate; the next-most-important components were gains in businesses and financial assets. In 2007, total unrealized capital gains in real estate represented 18.9 percent of total family assets. In general, the relative importance of unrealized capital gains in real estate decreases with family income and increases with the age of the family head.

\section{LIABILITIES}

The composition of household debt shifted between 2004 and 2007. Debt secured by the primary residence remained the largest component of overall household debt, but its share fell back 0.5 percentage point between the most recent surveys (table 11). ${ }^{42}$

This decline was more than offset by a 1.6 percentage point increase in the fraction of debt secured by residential property other than the primary residence. The share of outstanding credit card balances increased 0.5 percentage point over the three-year period, while the fraction of nonmortgage installment debt declined 0.8 percentage point, in line with a longer-term trend evident since at least the 1998 survey.

The overall value of families' liabilities increased between 2004 and 2007 at a rate just short of the corresponding rate for families' assets. Accordingly, the ratio of the sum of the debt of all families to the sum of their assets-the leverage ratio-was little changed, ticking down 0.1 percentage point, to 14.9 percent. The leverage ratio for the subset of

42. The SCF measure of liabilities excludes debt owed by businesses owned by the family and debt owed on nonresidential real estate.
12. Leverage ratio of group by selected family characteristics, 1998-2007 surveys

Percent

\begin{tabular}{|c|c|c|c|c|}
\hline Family characteristic & 1998 & 2001 & 2004 & 2007 \\
\hline All families & 14.2 & 12.1 & 15.0 & 14.9 \\
\hline \multicolumn{5}{|l|}{ Percentile of income } \\
\hline Less than $20 \ldots \ldots$. & 12.7 & 13.5 & 15.1 & 13.5 \\
\hline $20-39.9 \ldots \ldots$ & 14.4 & 14.5 & 19.4 & 18.5 \\
\hline $40-59.9$ & 20.6 & 19.2 & 23.2 & 24.3 \\
\hline $60-79.9$ & 23.1 & 18.0 & 21.7 & 25.3 \\
\hline $80-89.9$ & 20.1 & 18.1 & 22.8 & 23.4 \\
\hline $90-100$ & 8.9 & 7.4 & 9.2 & 8.4 \\
\hline \multicolumn{5}{|l|}{ Age of head (years) } \\
\hline Less than $35 \ldots \ldots$. & 36.6 & 33.5 & 46.4 & 44.3 \\
\hline $35-44 \ldots$ & 25.1 & 22.6 & 26.0 & 28.2 \\
\hline $45-54$ & 15.7 & 13.5 & 17.3 & 16.3 \\
\hline $55-64$ & 9.0 & 7.2 & 9.3 & 10.3 \\
\hline $65-74 \ldots \ldots$ & 4.7 & 4.2 & 5.2 & 6.5 \\
\hline 75 or more & 2.2 & 1.8 & 4.0 & 2.2 \\
\hline \multicolumn{5}{|l|}{ Education of head } \\
\hline No high school diploma & 13.5 & 13.4 & 14.0 & 18.2 \\
\hline High school diploma ... & 15.6 & 16.1 & 19.4 & 20.5 \\
\hline Some college ......... & 17.9 & 15.1 & 19.5 & 19.1 \\
\hline College degree & 12.9 & 10.4 & 13.3 & 12.5 \\
\hline \multicolumn{5}{|l|}{ Race or ethnicity of respondent } \\
\hline White non-Hispanic $\ldots \ldots \ldots \ldots$ & 13.3 & 11.0 & 13.5 & 12.9 \\
\hline Nonwhite or Hispanic & 23.5 & 23.4 & 27.2 & 27.1 \\
\hline \multicolumn{5}{|l|}{ Region } \\
\hline Northeast & 13.2 & 10.2 & 12.8 & 12.7 \\
\hline Midwest . & 14.1 & 13.0 & 14.4 & 14.4 \\
\hline South ... & 13.5 & 11.4 & 15.2 & 14.4 \\
\hline West ....... & 16.2 & 13.8 & 17.1 & 17.4 \\
\hline \multicolumn{5}{|l|}{ Urbanicity } \\
\hline Metropolitan statistical area (MSA) . & 14.2 & 12.0 & 14.8 & 14.7 \\
\hline Non-MSA $\ldots \ldots \ldots \ldots \ldots \ldots$ & 15.1 & 13.2 & 17.8 & 17.3 \\
\hline \multicolumn{5}{|l|}{ Housing status } \\
\hline Owner ................. & 14.1 & 12.0 & 14.9 & 14.7 \\
\hline Renter or other ........ & 16.4 & 14.2 & 16.7 & 17.9 \\
\hline \multicolumn{5}{|l|}{ Percentile of net worth } \\
\hline Less than $25 \ldots \ldots \ldots$ & 112.3 & 99.8 & 107.4 & 108.6 \\
\hline $25-49.9 \ldots$ & 51.0 & 47.9 & 54.2 & 56.5 \\
\hline $50-74.9$ & 27.1 & 26.2 & 33.3 & 31.7 \\
\hline $75-89.9$ & 16.1 & 14.4 & 16.3 & 17.6 \\
\hline $90-100$ & 5.9 & 4.8 & 6.4 & 6.1 \\
\hline
\end{tabular}

families that had any debt declined somewhat more, from 19.9 percent in 2004 to 19.4 percent in 2007 (data not shown in the tables).

The overall leverage ratio differs considerably across types of family groups. It rises and then falls across income groups. By comparison, the ratio declines with age, a result consistent with the expected life-cycle patterns of asset and debt accumulation. These general patterns in the leverage ratios among groups hold across survey years, but the variation among income groups was slightly more pronounced in 2007 than in 2004 (table 12).

\section{Holdings of Debt}

The share of families with any type of debt increased 0.6 percentage point, to 77.0 percent over the 2004-07 period (first half of tables 13.A and 13.B, last column), and has risen a total of 2.9 percentage points 
13. Family holdings of debt, by selected characteristics of families and type of debt, 2004 and 2007 surveys

A. 2004 Survey of Consumer Finances

\begin{tabular}{|c|c|c|c|c|c|c|c|}
\hline \multirow{2}{*}{ Family characteristic } & \multicolumn{2}{|c|}{ Secured by residential property } & \multirow{2}{*}{$\begin{array}{l}\text { Installment } \\
\text { loans }\end{array}$} & \multirow{2}{*}{$\begin{array}{c}\text { Credit card } \\
\text { balances }\end{array}$} & \multirow{2}{*}{$\begin{array}{l}\text { Lines of } \\
\text { credit not } \\
\text { secured by } \\
\text { residential } \\
\text { property }\end{array}$} & \multirow{2}{*}{ Other } & \multirow[b]{2}{*}{ Any deb } \\
\hline & $\begin{array}{c}\text { Primary } \\
\text { residence }\end{array}$ & Other & & & & & \\
\hline & \multicolumn{7}{|c|}{ Percentage of families holding debt } \\
\hline All families $\ldots \ldots \ldots \ldots \ldots$ & 47.9 & 4.0 & 46.0 & 46.2 & 1.6 & 7.6 & 76.4 \\
\hline 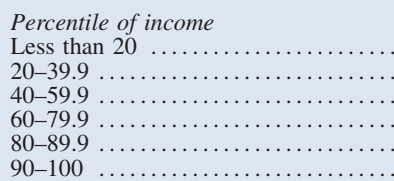 & $\begin{array}{l}15.9 \\
29.6 \\
51.6 \\
65.8 \\
76.8 \\
76.2\end{array}$ & $\begin{array}{r}* \\
1.5 \\
2.6 \\
4.1 \\
7.6 \\
15.4\end{array}$ & $\begin{array}{l}26.9 \\
39.8 \\
52.5 \\
57.9 \\
60.0 \\
45.7\end{array}$ & $\begin{array}{l}28.8 \\
42.9 \\
55.1 \\
56.1 \\
57.6 \\
38.5\end{array}$ & $\begin{array}{c}* \\
1.5 \\
1.8 \\
1.8 \\
2.6 \\
2.5\end{array}$ & $\begin{array}{r}4.6 \\
5.8 \\
8.0 \\
8.3 \\
12.2 \\
10.6\end{array}$ & $\begin{array}{l}52.6 \\
69.8 \\
84.0 \\
86.6 \\
91.9 \\
86.3\end{array}$ \\
\hline 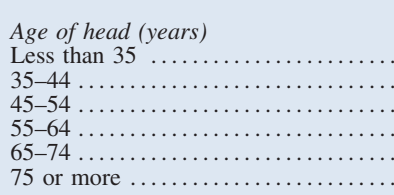 & $\begin{array}{l}37.7 \\
62.8 \\
64.6 \\
51.0 \\
32.1 \\
18.7\end{array}$ & $\begin{array}{l}2.1 \\
4.0 \\
6.3 \\
5.9 \\
3.2 \\
1.5\end{array}$ & $\begin{array}{l}59.4 \\
55.7 \\
50.2 \\
42.8 \\
27.5 \\
13.9\end{array}$ & $\begin{array}{l}47.5 \\
58.8 \\
54.0 \\
42.1 \\
31.9 \\
23.5\end{array}$ & $\begin{array}{r}2.2 \\
1.5 \\
2.9 \\
.7 \\
.4 \\
*\end{array}$ & $\begin{array}{r}6.2 \\
11.3 \\
9.4 \\
8.4 \\
4.0 \\
2.5\end{array}$ & $\begin{array}{l}79.8 \\
88.6 \\
88.4 \\
76.3 \\
58.8 \\
40.3\end{array}$ \\
\hline $\begin{array}{l}\text { Family structure } \\
\text { Single with child(ren) } \\
\text { Single, no child, age less than } 55 \ldots \ldots \\
\text { Single, no child, age } 55 \text { or more } \ldots \\
\text { Couple with child(ren) } \ldots \ldots \ldots \ldots \ldots \ldots \\
\text { Couple, no child } \ldots \ldots \ldots \ldots \ldots \ldots \ldots \ldots\end{array}$ & $\begin{array}{l}48.1 \\
34.1 \\
22.1 \\
64.1 \\
57.9\end{array}$ & $\begin{array}{l}1.5 \\
3.2 \\
2.5 \\
5.2 \\
5.0\end{array}$ & $\begin{array}{l}41.9 \\
46.4 \\
20.5 \\
61.2 \\
50.6\end{array}$ & $\begin{array}{l}48.7 \\
47.9 \\
27.9 \\
58.5 \\
47.5\end{array}$ & $\begin{array}{c}* \\
1.6 \\
* \\
2.2 \\
1.9\end{array}$ & $\begin{array}{l}6.7 \\
7.7 \\
5.0 \\
8.1 \\
8.4\end{array}$ & $\begin{array}{l}79.6 \\
77.6 \\
47.7 \\
87.8 \\
81.6\end{array}$ \\
\hline 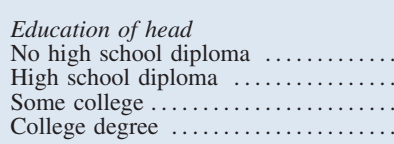 & $\begin{array}{l}24.8 \\
42.2 \\
48.7 \\
61.3\end{array}$ & $\begin{array}{l}* \\
2.2 \\
4.7 \\
6.7\end{array}$ & $\begin{array}{l}28.0 \\
44.3 \\
55.3 \\
49.9\end{array}$ & $\begin{array}{l}29.5 \\
48.2 \\
54.4 \\
47.0\end{array}$ & $\begin{array}{l}* \\
1.8 \\
1.8 \\
1.7\end{array}$ & $\begin{array}{r}5.7 \\
5.9 \\
10.3 \\
8.5\end{array}$ & $\begin{array}{l}53.4 \\
73.2 \\
84.2 \\
84.3\end{array}$ \\
\hline $\begin{array}{l}\text { Race or ethnicity of respondent } \\
\text { White non-Hispanic } \ldots \ldots \ldots \ldots \ldots \\
\text { Nonwhite or Hispanic } \ldots \ldots \ldots \ldots .\end{array}$ & $\begin{array}{l}51.9 \\
37.4\end{array}$ & $\begin{array}{l}4.4 \\
3.0\end{array}$ & $\begin{array}{l}47.0 \\
43.2\end{array}$ & $\begin{array}{l}46.0 \\
46.7\end{array}$ & $\begin{array}{l}1.7 \\
1.1\end{array}$ & $\begin{array}{l}7.8 \\
7.3\end{array}$ & $\begin{array}{l}78.0 \\
72.5\end{array}$ \\
\hline $\begin{array}{l}\text { Current work status of head } \\
\text { Working for someone else } \ldots . . \\
\text { Self-employed ............... } \\
\text { Retired ..................... } \\
\text { Other not working . . . . . . . . }\end{array}$ & $\begin{array}{l}56.1 \\
59.5 \\
24.6 \\
30.3\end{array}$ & $\begin{array}{r}4.1 \\
10.2 \\
1.2 \\
*\end{array}$ & $\begin{array}{l}55.7 \\
43.5 \\
22.8 \\
45.6\end{array}$ & $\begin{array}{l}54.9 \\
44.3 \\
25.9 \\
41.0\end{array}$ & $\begin{array}{l}1.9 \\
3.0 \\
* \\
*\end{array}$ & $\begin{array}{l}9.8 \\
5.8 \\
3.9 \\
*\end{array}$ & $\begin{array}{l}86.1 \\
81.5 \\
50.7 \\
70.4\end{array}$ \\
\hline $\begin{array}{l}\text { Current occupation of head } \\
\text { Managerial or professional ..... } \\
\text { Technical, sales, or services .... } \\
\text { Other occupation .............. } \\
\text { Retired or other not working ... }\end{array}$ & $\begin{array}{l}67.7 \\
45.7 \\
53.4 \\
25.5\end{array}$ & $\begin{array}{l}7.8 \\
3.4 \\
3.2 \\
1.3\end{array}$ & $\begin{array}{l}52.4 \\
52.5 \\
56.6 \\
26.3\end{array}$ & $\begin{array}{l}50.8 \\
54.2 \\
55.2 \\
28.2\end{array}$ & $\begin{array}{l}1.8 \\
2.4 \\
2.1 \\
*\end{array}$ & $\begin{array}{r}10.2 \\
7.5 \\
9.6 \\
3.6\end{array}$ & $\begin{array}{l}89.3 \\
81.5 \\
84.0 \\
53.7\end{array}$ \\
\hline 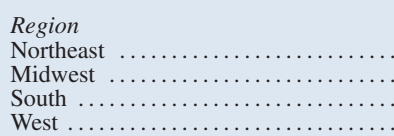 & $\begin{array}{l}47.4 \\
51.9 \\
45.2 \\
48.7\end{array}$ & $\begin{array}{l}3.5 \\
4.1 \\
3.2 \\
5.8\end{array}$ & $\begin{array}{l}42.4 \\
49.9 \\
44.2 \\
47.9\end{array}$ & $\begin{array}{l}46.6 \\
44.7 \\
46.0 \\
47.5\end{array}$ & $\begin{array}{l}1.1 \\
1.6 \\
1.6 \\
1.8\end{array}$ & $\begin{array}{l}7.8 \\
8.6 \\
6.5 \\
8.4\end{array}$ & $\begin{array}{l}76.3 \\
75.4 \\
75.0 \\
79.9\end{array}$ \\
\hline $\begin{array}{l}\text { Urbanicity } \\
\text { Metropolitan statistical area (MSA) ... } \\
\text { Non-MSA } \ldots \ldots \ldots \ldots \ldots \ldots \ldots \ldots \ldots \ldots \ldots \ldots \ldots \ldots\end{array}$ & $\begin{array}{l}49.0 \\
42.5\end{array}$ & $\begin{array}{l}4.4 \\
2.0\end{array}$ & $\begin{array}{l}45.4 \\
48.6\end{array}$ & $\begin{array}{l}46.9 \\
42.8\end{array}$ & $\begin{array}{l}1.6 \\
1.6\end{array}$ & $\begin{array}{l}7.9 \\
6.4\end{array}$ & $\begin{array}{l}76.8 \\
74.7\end{array}$ \\
\hline $\begin{array}{l}\text { Housing status } \\
\text { Owner } \ldots \ldots \ldots \ldots \ldots \ldots \ldots \ldots \ldots \ldots \ldots \ldots \ldots \ldots \ldots \ldots \ldots \ldots \ldots \\
\text { Renter or other } \ldots \ldots \ldots \ldots \ldots\end{array}$ & $\underset{*}{69.4}$ & $\begin{array}{l}5.1 \\
1.7\end{array}$ & $\begin{array}{l}46.6 \\
44.6\end{array}$ & $\begin{array}{l}48.8 \\
40.4\end{array}$ & $\begin{array}{l}1.3 \\
2.1\end{array}$ & $\begin{array}{l}7.7 \\
7.3\end{array}$ & $\begin{array}{l}82.3 \\
63.4\end{array}$ \\
\hline 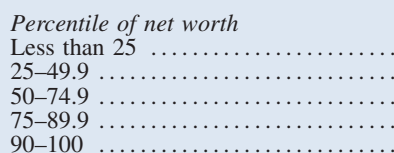 & $\begin{array}{l}12.4 \\
52.8 \\
66.1 \\
61.6 \\
58.4\end{array}$ & $\begin{array}{r}* \\
1.4 \\
4.5 \\
5.7 \\
16.6\end{array}$ & $\begin{array}{l}47.5 \\
52.4 \\
49.1 \\
40.2 \\
27.2\end{array}$ & $\begin{array}{l}40.3 \\
57.9 \\
52.8 \\
40.5 \\
23.4\end{array}$ & $\begin{array}{l}1.3 \\
1.7 \\
1.9 \\
1.3 \\
1.4\end{array}$ & $\begin{array}{l}6.2 \\
9.4 \\
7.0 \\
7.1 \\
9.1\end{array}$ & $\begin{array}{l}64.9 \\
83.8 \\
83.2 \\
74.6 \\
72.7\end{array}$ \\
\hline
\end{tabular}


13. Family holdings of debt, by selected characteristics of families and type of debt, 2004 and 2007 surveys-Continued A. 2004 Survey of Consumer Finances-continued

\begin{tabular}{|c|c|c|c|c|c|c|c|}
\hline \multirow{2}{*}{ Family characteristic } & \multicolumn{2}{|c|}{ Secured by residential property } & \multirow{2}{*}{$\begin{array}{l}\text { Installment } \\
\text { loans }\end{array}$} & \multirow{2}{*}{$\begin{array}{l}\text { Credit card } \\
\text { balances }\end{array}$} & \multirow{2}{*}{$\begin{array}{l}\text { Lines of } \\
\text { credit not } \\
\text { secured by } \\
\text { residential } \\
\text { property }\end{array}$} & \multirow{2}{*}{ Other } & \multirow{2}{*}{ Any debt } \\
\hline & $\begin{array}{c}\text { Primary } \\
\text { residence }\end{array}$ & Other & & & & & \\
\hline & \multicolumn{7}{|c|}{ Median value of holdings for families holding debt (thousands of 2007 dollars) } \\
\hline All families & 104.3 & 95.6 & 12.7 & 2.4 & 3.3 & 4.4 & 60.7 \\
\hline 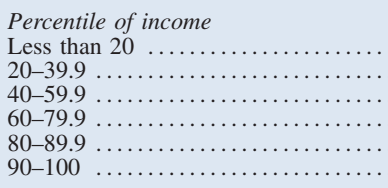 & $\begin{array}{r}40.6 \\
59.3 \\
84.8 \\
106.5 \\
146.1 \\
203.2\end{array}$ & $\begin{array}{r}* \\
35.7 \\
72.5 \\
68.1 \\
85.7 \\
174.6\end{array}$ & $\begin{array}{r}6.1 \\
8.8 \\
11.8 \\
15.2 \\
16.6 \\
19.8\end{array}$ & $\begin{array}{l}1.1 \\
2.0 \\
2.4 \\
3.3 \\
3.0 \\
4.4\end{array}$ & $\begin{array}{r}* \\
.3 \\
1.1 \\
7.7 \\
15.4 \\
43.9\end{array}$ & $\begin{array}{r}2.2 \\
2.9 \\
2.5 \\
3.8 \\
5.5 \\
10.4\end{array}$ & $\begin{array}{r}7.7 \\
17.6 \\
48.8 \\
102.6 \\
149.4 \\
229.5\end{array}$ \\
\hline 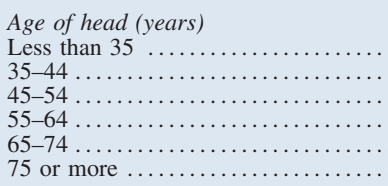 & $\begin{array}{r}117.5 \\
120.8 \\
106.5 \\
91.2 \\
56.0 \\
34.0\end{array}$ & $\begin{array}{r}68.6 \\
82.4 \\
95.6 \\
119.5 \\
109.8 \\
42.8\end{array}$ & $\begin{array}{r}13.1 \\
13.2 \\
13.1 \\
14.2 \\
9.1 \\
7.4\end{array}$ & $\begin{array}{l}1.6 \\
2.7 \\
3.2 \\
2.4 \\
2.4 \\
1.1\end{array}$ & $\begin{array}{r}1.1 \\
2.1 \\
7.7 \\
15.4 \\
4.4 \\
*\end{array}$ & $\begin{array}{l}3.3 \\
4.4 \\
4.4 \\
6.0 \\
5.5 \\
2.2\end{array}$ & $\begin{array}{l}36.9 \\
95.8 \\
91.4 \\
52.7 \\
27.5 \\
16.9\end{array}$ \\
\hline 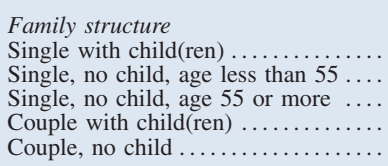 & $\begin{array}{r}71.4 \\
97.7 \\
52.7 \\
104.3 \\
119.7\end{array}$ & $\begin{array}{r}71.4 \\
72.5 \\
87.9 \\
153.8 \\
104.3\end{array}$ & $\begin{array}{r}9.3 \\
9.8 \\
8.8 \\
14.6 \\
15.0\end{array}$ & $\begin{array}{l}2.4 \\
2.1 \\
2.0 \\
3.3 \\
2.3\end{array}$ & $\begin{array}{l}* \\
1.1 \\
* \\
4.4 \\
9.9\end{array}$ & $\begin{array}{l}2.2 \\
3.3 \\
2.2 \\
4.9 \\
5.5\end{array}$ & $\begin{array}{l}44.0 \\
23.4 \\
18.1 \\
97.0 \\
93.4\end{array}$ \\
\hline $\begin{array}{l}\text { Education of head } \\
\text { No high school diploma } \ldots \ldots \ldots \\
\text { High school diploma } \ldots \ldots \ldots \ldots \\
\text { Some college } \ldots \ldots \ldots \ldots \ldots \ldots \ldots \\
\text { College degree } \ldots \ldots \ldots \ldots \ldots \ldots \ldots\end{array}$ & $\begin{array}{r}48.3 \\
76.9 \\
94.5 \\
137.3\end{array}$ & $\begin{array}{c}* \\
51.6 \\
82.4 \\
115.3\end{array}$ & $\begin{array}{r}7.7 \\
9.9 \\
13.0 \\
16.9\end{array}$ & $\begin{array}{l}1.3 \\
2.1 \\
2.4 \\
3.0\end{array}$ & $\begin{array}{l}* \\
1.6 \\
3.3 \\
4.4\end{array}$ & $\begin{array}{l}4.4 \\
3.3 \\
3.7 \\
5.5\end{array}$ & $\begin{array}{r}13.2 \\
34.0 \\
49.4 \\
117.7\end{array}$ \\
\hline $\begin{array}{l}\text { Race or ethnicity of respondent } \\
\text { White non-Hispanic } \ldots \ldots \ldots \ldots \ldots \\
\text { Nonwhite or Hispanic } \ldots \ldots \ldots \ldots\end{array}$ & $\begin{array}{r}107.6 \\
91.2\end{array}$ & $\begin{array}{l}95.6 \\
72.5\end{array}$ & $\begin{array}{l}13.7 \\
10.5\end{array}$ & $\begin{array}{l}2.7 \\
1.7\end{array}$ & $\begin{array}{r}4.4 \\
.4\end{array}$ & $\begin{array}{l}4.4 \\
3.3\end{array}$ & $\begin{array}{l}76.3 \\
33.5\end{array}$ \\
\hline $\begin{array}{l}\text { Current work status of head } \\
\text { Working for someone else ... } \\
\text { Self-employed .............. } \\
\text { Retired .................. } \\
\text { Other not working } \ldots \ldots \ldots \ldots \ldots\end{array}$ & $\begin{array}{r}109.8 \\
131.6 \\
46.1 \\
85.7\end{array}$ & $\begin{array}{r}91.2 \\
109.8 \\
86.8 \\
*\end{array}$ & $\begin{array}{r}13.2 \\
16.9 \\
8.0 \\
8.2\end{array}$ & $\begin{array}{l}2.5 \\
3.0 \\
1.6 \\
2.7\end{array}$ & $\begin{array}{l}4.4 \\
2.4 \\
* \\
*\end{array}$ & $\begin{array}{l}3.8 \\
7.7 \\
3.3 \\
*\end{array}$ & $\begin{array}{r}78.9 \\
102.6 \\
16.9 \\
23.1\end{array}$ \\
\hline $\begin{array}{l}\text { Current occupation of head } \\
\text { Managerial or professional .... } \\
\text { Technical, sales, or services .. } \\
\text { Other occupation ............ } \\
\text { Retired or other not working . }\end{array}$ & $\begin{array}{r}141.7 \\
97.7 \\
90.8 \\
54.9\end{array}$ & $\begin{array}{r}101.0 \\
115.3 \\
85.7 \\
106.5\end{array}$ & $\begin{array}{r}16.5 \\
12.2 \\
11.3 \\
8.2\end{array}$ & $\begin{array}{l}3.3 \\
2.2 \\
2.5 \\
1.6\end{array}$ & $\begin{array}{l}8.8 \\
1.6 \\
1.6 \\
*\end{array}$ & $\begin{array}{l}5.5 \\
3.3 \\
3.3 \\
4.4\end{array}$ & $\begin{array}{r}127.3 \\
47.6 \\
56.4 \\
17.7\end{array}$ \\
\hline $\begin{array}{l}\text { Region } \\
\text { Northeast } \ldots \ldots \ldots \ldots \ldots \ldots \\
\text { Midwest } \ldots \ldots \ldots \ldots \ldots \ldots \ldots \\
\text { South } \ldots \ldots \ldots \ldots \ldots \ldots \ldots \ldots \\
\text { West } \ldots \ldots \ldots \ldots \ldots \ldots \ldots \ldots \ldots \ldots\end{array}$ & $\begin{array}{r}122.5 \\
94.5 \\
86.8 \\
140.7\end{array}$ & $\begin{array}{r}109.8 \\
87.9 \\
91.2 \\
95.6\end{array}$ & $\begin{array}{l}13.0 \\
12.1 \\
12.3 \\
14.1\end{array}$ & $\begin{array}{l}2.7 \\
2.2 \\
2.2 \\
2.7\end{array}$ & $\begin{array}{r}.4 \\
3.3 \\
8.8 \\
4.4\end{array}$ & $\begin{array}{l}5.5 \\
4.4 \\
4.4 \\
3.3\end{array}$ & $\begin{array}{l}60.1 \\
75.4 \\
44.3 \\
85.2\end{array}$ \\
\hline $\begin{array}{l}\text { Urbanicity } \\
\text { Metropolitan statistical area (MSA) .. } \\
\text { Non-MSA } \ldots \ldots \ldots \ldots \ldots \ldots \ldots \ldots \ldots \ldots \ldots \ldots \ldots \ldots \ldots \ldots\end{array}$ & $\begin{array}{r}115.3 \\
54.9\end{array}$ & $\begin{array}{l}96.6 \\
69.2\end{array}$ & $\begin{array}{l}13.2 \\
10.9\end{array}$ & $\begin{array}{l}2.4 \\
2.2\end{array}$ & $\begin{array}{r}2.4 \\
22.0\end{array}$ & $\begin{array}{l}4.4 \\
4.4\end{array}$ & $\begin{array}{l}75.8 \\
28.9\end{array}$ \\
\hline $\begin{array}{l}\text { Housing status } \\
\text { Owner .............. } \\
\text { Renter or other ........ }\end{array}$ & $\begin{array}{c}104.3 \\
*\end{array}$ & $\begin{array}{l}98.8 \\
91.2\end{array}$ & $\begin{array}{r}14.2 \\
9.6\end{array}$ & $\begin{array}{l}2.7 \\
1.6\end{array}$ & $\begin{array}{r}8.8 \\
.5\end{array}$ & $\begin{array}{l}4.4 \\
3.3\end{array}$ & $\begin{array}{r}105.2 \\
8.6\end{array}$ \\
\hline 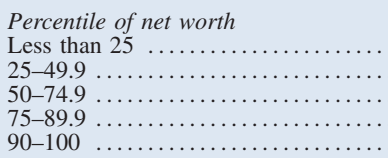 & $\begin{array}{r}78.0 \\
82.4 \\
106.5 \\
126.3 \\
204.4\end{array}$ & $\begin{array}{r}* \\
28.9 \\
51.6 \\
108.7 \\
162.5\end{array}$ & $\begin{array}{l}11.5 \\
10.2 \\
14.6 \\
14.2 \\
19.2\end{array}$ & $\begin{array}{l}1.9 \\
2.2 \\
2.7 \\
3.3 \\
3.3\end{array}$ & $\begin{array}{r}.3 \\
1.1 \\
8.8 \\
24.2 \\
54.9\end{array}$ & $\begin{array}{r}4.4 \\
2.2 \\
4.4 \\
5.5 \\
22.0\end{array}$ & $\begin{array}{r}12.5 \\
48.6 \\
98.9 \\
121.6 \\
209.5\end{array}$ \\
\hline $\begin{array}{l}\text { MEмO } \\
\text { Mean value of holdings for } \\
\text { families holding asset } . . .\end{array}$ & 136.2 & 183.1 & 20.7 & 5.6 & 40.2 & 18.7 & 113.5 \\
\hline
\end{tabular}

NotE: See note to table 11.

* Ten or fewer observations. 
13. Family holdings of debt, by selected characteristics of families and type of debt, 2004-2007 surveys-Continued B. 2007 Survey of Consumer Finances

\begin{tabular}{|c|c|c|c|c|c|c|c|}
\hline \multirow{2}{*}{ Family characteristic } & \multicolumn{2}{|c|}{ Secured by residential property } & \multirow{2}{*}{$\begin{array}{l}\text { Installment } \\
\text { loans }\end{array}$} & \multirow{2}{*}{$\begin{array}{c}\text { Credit card } \\
\text { balances }\end{array}$} & \multirow{2}{*}{$\begin{array}{l}\text { Lines of } \\
\text { credit not } \\
\text { secured by } \\
\text { residential } \\
\text { property }\end{array}$} & \multirow{2}{*}{ Other } & \multirow{2}{*}{ Any deb } \\
\hline & $\begin{array}{l}\text { Primary } \\
\text { residence }\end{array}$ & Other & & & & & \\
\hline & \multicolumn{7}{|c|}{ Percentage of families holding debt } \\
\hline All families $\ldots \ldots \ldots \ldots \ldots \ldots \ldots$ & 48.7 & 5.5 & 46.9 & 46.1 & 1.7 & 6.8 & 77.0 \\
\hline 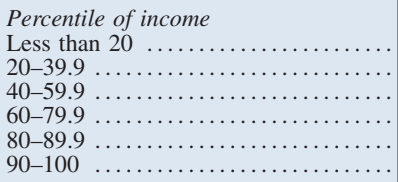 & $\begin{array}{l}14.9 \\
29.5 \\
50.5 \\
69.7 \\
80.8 \\
76.4\end{array}$ & $\begin{array}{r}1.1 \\
1.9 \\
2.6 \\
6.8 \\
8.5 \\
21.9\end{array}$ & $\begin{array}{l}27.8 \\
42.3 \\
54.0 \\
59.2 \\
57.4 \\
45.0\end{array}$ & $\begin{array}{l}25.7 \\
39.4 \\
54.9 \\
62.1 \\
55.8 \\
40.6\end{array}$ & $\begin{array}{c}* \\
1.8 \\
* \\
2.1 \\
* \\
2.1\end{array}$ & $\begin{array}{l}3.9 \\
6.8 \\
6.4 \\
8.7 \\
9.6 \\
7.0\end{array}$ & $\begin{array}{l}51.7 \\
70.2 \\
83.8 \\
90.9 \\
89.6 \\
87.6\end{array}$ \\
\hline 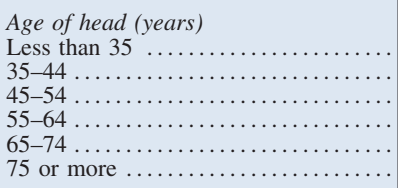 & $\begin{array}{l}37.3 \\
59.5 \\
65.5 \\
55.3 \\
42.9 \\
13.9\end{array}$ & $\begin{array}{r}3.3 \\
6.5 \\
8.0 \\
7.8 \\
5.0 \\
.6\end{array}$ & $\begin{array}{r}65.2 \\
56.2 \\
51.9 \\
44.6 \\
26.1 \\
7.0\end{array}$ & $\begin{array}{l}48.5 \\
51.7 \\
53.6 \\
49.9 \\
37.0 \\
18.8\end{array}$ & $\begin{array}{l}2.1 \\
2.2 \\
1.9 \\
1.2 \\
1.5 \\
*\end{array}$ & $\begin{array}{l}5.9 \\
7.5 \\
9.8 \\
8.7 \\
4.4 \\
1.3\end{array}$ & $\begin{array}{l}83.5 \\
86.2 \\
86.8 \\
81.8 \\
65.5 \\
31.4\end{array}$ \\
\hline $\begin{array}{l}\text { Family structure } \\
\text { Single with child(ren) } \ldots \ldots \ldots \ldots \ldots \\
\text { Single, no child, age less than } 55 \ldots \ldots \\
\text { Single, no child, age } 55 \text { or more } \ldots \ldots \\
\text { Couple with child(ren) } \ldots \ldots \ldots \ldots \ldots \ldots \\
\text { Couple, no child } \ldots \ldots \ldots \ldots \ldots \ldots \ldots \ldots\end{array}$ & $\begin{array}{l}38.0 \\
35.6 \\
23.2 \\
67.0 \\
59.1\end{array}$ & $\begin{array}{l}3.5 \\
3.0 \\
1.8 \\
6.9 \\
7.7\end{array}$ & $\begin{array}{l}48.3 \\
46.7 \\
19.4 \\
63.9 \\
51.4\end{array}$ & $\begin{array}{l}45.6 \\
43.5 \\
30.5 \\
55.7 \\
49.8\end{array}$ & $\begin{array}{c}* \\
2.0 \\
* \\
1.9 \\
1.7\end{array}$ & $\begin{array}{r}11.1 \\
7.4 \\
4.1 \\
6.5 \\
7.0\end{array}$ & $\begin{array}{l}81.6 \\
76.1 \\
49.0 \\
90.4 \\
82.5\end{array}$ \\
\hline $\begin{array}{l}\text { Education of head } \\
\text { No high school diploma } \ldots \ldots \ldots \ldots \ldots \\
\text { High school diploma } \ldots \ldots \ldots \ldots \ldots \ldots \\
\text { Some college } \ldots \ldots \ldots \ldots \ldots \ldots \ldots \ldots \\
\text { College degree } \ldots \ldots \ldots \ldots \ldots \ldots \ldots \ldots\end{array}$ & $\begin{array}{l}26.0 \\
45.0 \\
46.9 \\
61.6\end{array}$ & $\begin{array}{l}1.9 \\
3.2 \\
6.4 \\
8.7\end{array}$ & $\begin{array}{l}33.3 \\
46.0 \\
54.3 \\
49.1\end{array}$ & $\begin{array}{l}26.9 \\
46.8 \\
51.0 \\
50.2\end{array}$ & $\begin{array}{l}* \\
1.4 \\
2.2 \\
1.7\end{array}$ & $\begin{array}{l}5.3 \\
6.4 \\
9.3 \\
6.5\end{array}$ & $\begin{array}{l}55.5 \\
75.1 \\
80.8 \\
85.1\end{array}$ \\
\hline $\begin{array}{l}\text { Race or ethnicity of respondent } \\
\text { White non-Hispanic } \ldots \ldots \ldots \ldots \ldots \ldots \\
\text { Nonwhite or Hispanic } \ldots \ldots \ldots \ldots \ldots \ldots\end{array}$ & $\begin{array}{l}52.1 \\
40.4\end{array}$ & $\begin{array}{l}5.8 \\
4.8\end{array}$ & $\begin{array}{l}46.1 \\
48.9\end{array}$ & $\begin{array}{l}45.1 \\
48.4\end{array}$ & $\begin{array}{l}1.6 \\
2.0\end{array}$ & $\begin{array}{l}6.7 \\
7.0\end{array}$ & $\begin{array}{l}76.8 \\
77.7\end{array}$ \\
\hline $\begin{array}{l}\text { Current work status of head } \\
\text { Working for someone else } \ldots \ldots \ldots \ldots \\
\text { Self-employed } \ldots \ldots \ldots \ldots \ldots \ldots \ldots \ldots \ldots \\
\text { Retired } \ldots \ldots \ldots \ldots \ldots \ldots \ldots \ldots \ldots \ldots \\
\text { Other not working } \ldots \ldots \ldots \ldots \ldots \ldots \ldots\end{array}$ & $\begin{array}{l}56.7 \\
64.8 \\
27.0 \\
25.4\end{array}$ & $\begin{array}{r}5.4 \\
15.1 \\
2.6 \\
*\end{array}$ & $\begin{array}{l}57.5 \\
43.9 \\
23.6 \\
42.8\end{array}$ & $\begin{array}{l}53.7 \\
48.9 \\
28.2 \\
36.8\end{array}$ & $\begin{array}{c}1.9 \\
3.6 \\
.8 \\
*\end{array}$ & $\begin{array}{l}8.7 \\
4.7 \\
3.2 \\
7.5\end{array}$ & $\begin{array}{l}86.2 \\
86.8 \\
52.3 \\
69.7\end{array}$ \\
\hline $\begin{array}{l}\text { Current occupation of head } \\
\text { Managerial or professional } \ldots \ldots \ldots \ldots \\
\text { Technical, sales, or services } \ldots \ldots \ldots \ldots \\
\text { Other occupation } \ldots \ldots \ldots \ldots \ldots \ldots \\
\text { Retired or other not working } \ldots \ldots \ldots \ldots\end{array}$ & $\begin{array}{l}67.6 \\
49.7 \\
53.6 \\
26.7\end{array}$ & $\begin{array}{r}10.0 \\
4.5 \\
5.1 \\
2.5\end{array}$ & $\begin{array}{l}56.2 \\
52.2 \\
57.8 \\
26.6\end{array}$ & $\begin{array}{l}52.7 \\
53.2 \\
53.2 \\
29.6\end{array}$ & $\begin{array}{r}1.8 \\
2.7 \\
2.1 \\
.7\end{array}$ & $\begin{array}{l}7.0 \\
7.9 \\
9.7 \\
3.9\end{array}$ & $\begin{array}{l}90.9 \\
81.8 \\
84.9 \\
55.0\end{array}$ \\
\hline $\begin{array}{l}\text { Region } \\
\text { Northeast } \ldots \ldots \ldots \ldots \ldots \ldots \ldots \ldots \ldots \\
\text { Midwest } \ldots \ldots \ldots \ldots \ldots \ldots \ldots \ldots \ldots \ldots \\
\text { South } \ldots \ldots \ldots \ldots \ldots \ldots \ldots \ldots \ldots \ldots \ldots \\
\text { West } \ldots \ldots \ldots \ldots \ldots \ldots \ldots \ldots \ldots \ldots\end{array}$ & $\begin{array}{l}48.4 \\
51.0 \\
46.6 \\
49.9\end{array}$ & $\begin{array}{l}4.9 \\
5.2 \\
4.6 \\
8.1\end{array}$ & $\begin{array}{l}40.7 \\
47.9 \\
48.5 \\
48.4\end{array}$ & $\begin{array}{l}44.3 \\
45.5 \\
43.4 \\
52.4\end{array}$ & $\begin{array}{l}* \\
1.9 \\
1.7 \\
2.7\end{array}$ & $\begin{array}{l}5.6 \\
7.0 \\
6.9 \\
7.5\end{array}$ & $\begin{array}{l}73.3 \\
78.3 \\
75.3 \\
81.6\end{array}$ \\
\hline $\begin{array}{l}\text { Urbanicity } \\
\text { Metropolitan statistical area (MSA) } \ldots \\
\text { Non-MSA } \ldots \ldots \ldots \ldots \ldots \ldots \ldots \ldots\end{array}$ & $\begin{array}{l}49.7 \\
43.5\end{array}$ & $\begin{array}{l}6.1 \\
2.9\end{array}$ & $\begin{array}{l}46.0 \\
51.2\end{array}$ & $\begin{array}{l}46.3 \\
44.8\end{array}$ & $\begin{array}{l}1.8 \\
1.6\end{array}$ & $\begin{array}{l}6.6 \\
8.0\end{array}$ & $\begin{array}{l}77.4 \\
75.0\end{array}$ \\
\hline $\begin{array}{l}\text { Housing status } \\
\text { Owner } \ldots \ldots \ldots \ldots \ldots \ldots \ldots \ldots \ldots \ldots \ldots \ldots \ldots \ldots \ldots \ldots \\
\text { Renter or other } \ldots \ldots \ldots \ldots \ldots \ldots \ldots\end{array}$ & $\begin{array}{c}70.9 \\
*\end{array}$ & $\begin{array}{l}6.9 \\
2.6\end{array}$ & $\begin{array}{l}46.1 \\
48.6\end{array}$ & $\begin{array}{l}50.1 \\
37.3\end{array}$ & $\begin{array}{l}1.3 \\
2.8\end{array}$ & $\begin{array}{l}6.8 \\
6.9\end{array}$ & $\begin{array}{l}82.4 \\
65.4\end{array}$ \\
\hline 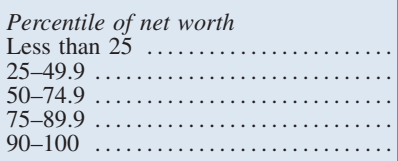 & $\begin{array}{l}11.0 \\
56.1 \\
64.3 \\
63.9 \\
62.1\end{array}$ & $\begin{array}{r}* \\
3.2 \\
4.8 \\
8.5 \\
21.8\end{array}$ & $\begin{array}{l}54.2 \\
52.1 \\
46.1 \\
39.8 \\
28.2\end{array}$ & $\begin{array}{l}41.0 \\
52.9 \\
51.7 \\
44.1 \\
30.3\end{array}$ & $\begin{array}{l}2.6 \\
1.3 \\
1.6 \\
1.5 \\
1.5\end{array}$ & $\begin{array}{l}6.7 \\
8.2 \\
7.4 \\
3.8 \\
6.7\end{array}$ & $\begin{array}{l}68.9 \\
82.4 \\
80.3 \\
76.9 \\
75.9\end{array}$ \\
\hline
\end{tabular}


13. Family holdings of debt, by selected characteristics of families and type of debt, 2004-2007 surveys-Continued

B. 2007 Survey of Consumer Finances-continued

\begin{tabular}{|c|c|c|c|c|c|c|c|}
\hline \multirow{2}{*}{ Family characteristic } & \multicolumn{2}{|c|}{ Secured by residential property } & \multirow{2}{*}{$\begin{array}{l}\text { Installment } \\
\text { loans }\end{array}$} & \multirow{2}{*}{$\begin{array}{l}\text { Credit card } \\
\text { balances }\end{array}$} & \multirow{2}{*}{$\begin{array}{l}\text { Lines of } \\
\text { credit not } \\
\text { secured by } \\
\text { residential } \\
\text { property }\end{array}$} & \multirow{2}{*}{ Other } & \multirow{2}{*}{ Any deb } \\
\hline & $\begin{array}{c}\text { Primary } \\
\text { residence }\end{array}$ & Other & & & & & \\
\hline & \multicolumn{7}{|c|}{ Median value of holdings for families holding debt (thousands of 2007 dollars) } \\
\hline All families & 107.0 & 100.0 & 13.0 & 3.0 & 3.8 & 5.0 & 67.3 \\
\hline 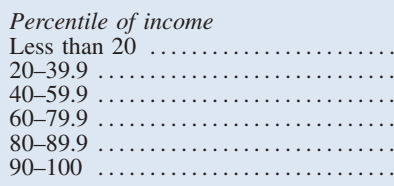 & $\begin{array}{r}40.0 \\
51.0 \\
88.7 \\
115.0 \\
164.0 \\
201.0\end{array}$ & $\begin{array}{r}70.0 \\
42.0 \\
68.9 \\
83.0 \\
125.0 \\
147.5\end{array}$ & $\begin{array}{r}6.5 \\
9.8 \\
12.8 \\
16.3 \\
17.3 \\
18.3\end{array}$ & $\begin{array}{l}1.0 \\
1.8 \\
2.4 \\
4.0 \\
5.5 \\
7.5\end{array}$ & $\begin{array}{c}* \\
1.3 \\
* \\
5.1 \\
* \\
17.3\end{array}$ & $\begin{array}{l}3.0 \\
4.0 \\
4.0 \\
5.3 \\
5.0 \\
7.5\end{array}$ & $\begin{array}{r}9.0 \\
18.0 \\
54.5 \\
111.3 \\
182.2 \\
235.0\end{array}$ \\
\hline 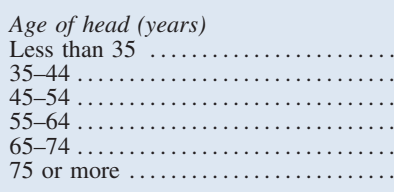 & $\begin{array}{r}135.3 \\
128.0 \\
110.0 \\
85.0 \\
69.0 \\
40.0\end{array}$ & $\begin{array}{r}78.0 \\
101.6 \\
82.0 \\
130.0 \\
125.0 \\
50.0\end{array}$ & $\begin{array}{r}15.0 \\
13.5 \\
12.9 \\
10.9 \\
10.3 \\
8.0\end{array}$ & $\begin{array}{r}1.8 \\
3.5 \\
3.6 \\
3.6 \\
3.0 \\
.8\end{array}$ & $\begin{array}{r}1.0 \\
4.6 \\
6.0 \\
10.0 \\
30.0 \\
*\end{array}$ & $\begin{array}{l}4.5 \\
5.0 \\
4.5 \\
6.0 \\
5.0 \\
4.5\end{array}$ & $\begin{array}{r}36.2 \\
106.2 \\
95.9 \\
60.3 \\
40.1 \\
13.0\end{array}$ \\
\hline 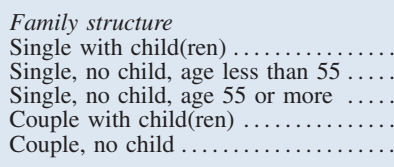 & $\begin{array}{r}97.0 \\
93.9 \\
50.0 \\
119.0 \\
119.0\end{array}$ & $\begin{array}{r}92.5 \\
80.0 \\
135.0 \\
114.8 \\
100.0\end{array}$ & $\begin{array}{r}10.0 \\
10.0 \\
6.0 \\
13.0 \\
15.8\end{array}$ & $\begin{array}{l}2.0 \\
1.5 \\
2.3 \\
4.1 \\
3.5\end{array}$ & $\begin{array}{l}* \\
.4 \\
* \\
3.5 \\
5.1\end{array}$ & $\begin{array}{l}7.0 \\
4.5 \\
3.8 \\
6.0 \\
5.0\end{array}$ & $\begin{array}{r}27.9 \\
31.0 \\
15.9 \\
103.0 \\
102.7\end{array}$ \\
\hline 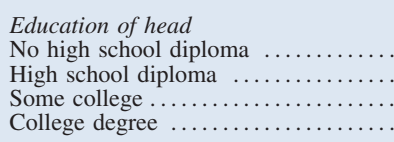 & $\begin{array}{r}50.0 \\
84.0 \\
97.0 \\
142.7\end{array}$ & $\begin{array}{r}53.3 \\
82.0 \\
80.0 \\
125.0\end{array}$ & $\begin{array}{r}8.8 \\
10.2 \\
12.1 \\
17.4\end{array}$ & $\begin{array}{l}1.5 \\
2.3 \\
2.9 \\
4.0\end{array}$ & $\begin{array}{l}* \\
1.4 \\
3.8 \\
6.0\end{array}$ & $\begin{array}{l}4.0 \\
4.5 \\
5.0 \\
6.0\end{array}$ & $\begin{array}{r}19.5 \\
40.0 \\
54.4 \\
124.3\end{array}$ \\
\hline $\begin{array}{l}\text { Race or ethnicity of respondent } \\
\text { White non-Hispanic . .......... } \\
\text { Nonwhite or Hispanic ......... }\end{array}$ & $\begin{array}{l}106.0 \\
113.0\end{array}$ & $\begin{array}{r}90.8 \\
114.8\end{array}$ & $\begin{array}{l}13.4 \\
12.0\end{array}$ & $\begin{array}{l}3.3 \\
2.0\end{array}$ & $\begin{array}{r}5.0 \\
.8\end{array}$ & $\begin{array}{l}5.0 \\
5.0\end{array}$ & $\begin{array}{l}76.4 \\
43.9\end{array}$ \\
\hline 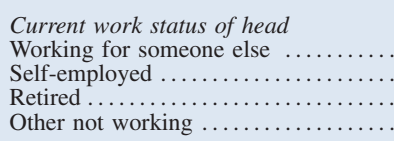 & $\begin{array}{r}117.0 \\
135.0 \\
47.1 \\
90.0\end{array}$ & $\begin{array}{r}89.0 \\
151.6 \\
100.0 \\
*\end{array}$ & $\begin{array}{r}13.5 \\
15.5 \\
8.6 \\
10.7\end{array}$ & $\begin{array}{l}3.0 \\
4.3 \\
1.5 \\
1.8\end{array}$ & $\begin{array}{l}2.9 \\
5.0 \\
6.4 \\
*\end{array}$ & $\begin{array}{r}5.0 \\
10.0 \\
4.5 \\
8.0\end{array}$ & $\begin{array}{r}82.1 \\
122.7 \\
20.0 \\
21.9\end{array}$ \\
\hline 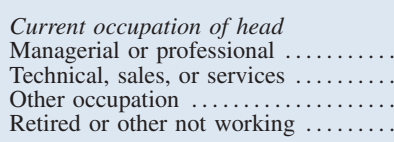 & $\begin{array}{r}148.0 \\
100.9 \\
94.0 \\
53.0\end{array}$ & $\begin{array}{r}130.0 \\
105.0 \\
60.0 \\
100.0\end{array}$ & $\begin{array}{r}16.3 \\
12.2 \\
12.0 \\
9.7\end{array}$ & $\begin{array}{l}4.5 \\
3.0 \\
2.5 \\
1.5\end{array}$ & $\begin{array}{l}9.0 \\
3.5 \\
4.0 \\
6.4\end{array}$ & $\begin{array}{l}7.0 \\
4.0 \\
4.8 \\
5.0\end{array}$ & $\begin{array}{r}137.6 \\
65.8 \\
64.1 \\
20.0\end{array}$ \\
\hline 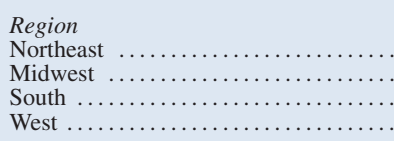 & $\begin{array}{r}107.0 \\
93.9 \\
99.0 \\
150.8\end{array}$ & $\begin{array}{r}95.0 \\
82.5 \\
80.0 \\
160.0\end{array}$ & $\begin{array}{l}12.1 \\
11.0 \\
13.2 \\
14.2\end{array}$ & $\begin{array}{l}3.0 \\
3.0 \\
2.8 \\
3.0\end{array}$ & $\begin{array}{l}* \\
5.0 \\
3.2 \\
3.8\end{array}$ & $\begin{array}{l}6.5 \\
5.0 \\
4.5 \\
6.0\end{array}$ & $\begin{array}{l}66.6 \\
61.2 \\
60.9 \\
95.5\end{array}$ \\
\hline $\begin{array}{l}\text { Urbanicity } \\
\text { Metropolitan statistical area (MSA) ... } \\
\text { Non-MSA } \ldots \ldots \ldots \ldots \ldots \ldots \ldots \ldots \ldots \ldots \ldots \ldots \ldots \ldots\end{array}$ & $\begin{array}{r}118.2 \\
60.7\end{array}$ & $\begin{array}{r}101.0 \\
70.0\end{array}$ & $\begin{array}{l}13.3 \\
11.7\end{array}$ & $\begin{array}{l}3.0 \\
2.0\end{array}$ & $\begin{array}{l}3.5 \\
6.0\end{array}$ & $\begin{array}{l}5.0 \\
5.0\end{array}$ & $\begin{array}{l}78.1 \\
29.8\end{array}$ \\
\hline $\begin{array}{l}\text { Housing status } \\
\text { Owner } \ldots \ldots \ldots \ldots \ldots \ldots \ldots \\
\text { Renter or other } \ldots \ldots \ldots \ldots \ldots \ldots\end{array}$ & $\begin{array}{c}107.0 \\
*\end{array}$ & $\begin{array}{r}100.0 \\
80.0\end{array}$ & $\begin{array}{l}14.2 \\
10.3\end{array}$ & $\begin{array}{l}3.6 \\
1.3\end{array}$ & $\begin{array}{l}7.5 \\
1.0\end{array}$ & $\begin{array}{l}5.0 \\
4.9\end{array}$ & $\begin{array}{r}111.1 \\
9.2\end{array}$ \\
\hline $\begin{array}{l}\text { Percentile of net worth } \\
\text { Less than } 25 \ldots \ldots \ldots \ldots \ldots \ldots \\
25-49.9 \ldots \ldots \ldots \ldots \ldots \ldots \ldots \ldots \\
50-74.9 \ldots \ldots \ldots \ldots \ldots \ldots \ldots \ldots \ldots \ldots \\
75-99.9 \ldots \ldots \ldots \ldots \ldots \ldots \ldots \ldots \ldots \ldots \ldots\end{array}$ & $\begin{array}{r}107.0 \\
85.0 \\
104.0 \\
130.0 \\
180.0\end{array}$ & $\begin{array}{r}* \\
74.0 \\
72.0 \\
94.0 \\
160.0\end{array}$ & $\begin{array}{l}11.4 \\
13.0 \\
14.0 \\
12.0 \\
17.1\end{array}$ & $\begin{array}{l}1.5 \\
2.8 \\
3.5 \\
4.0 \\
4.5\end{array}$ & $\begin{array}{r}1.0 \\
2.0 \\
4.2 \\
10.3 \\
43.0\end{array}$ & $\begin{array}{r}5.0 \\
3.9 \\
5.0 \\
5.0 \\
15.0\end{array}$ & $\begin{array}{r}11.9 \\
64.2 \\
97.5 \\
127.0 \\
203.0\end{array}$ \\
\hline $\begin{array}{l}\text { MEMO } \\
\text { Mean value of holdings for } \\
\text { families holding asset } \ldots\end{array}$ & 149.0 & 177.3 & 21.0 & 7.3 & 24.8 & 15.5 & 126.0 \\
\hline
\end{tabular}

NotE: See note to table 11.

* Ten or fewer observations. 
since the 1998 survey (data not shown in the tables). In general, borrowing is less prevalent among childless single families headed by a person aged 55 or older and families headed by a person who is retired or is 75 or older. Families in the lowest income, wealth, and education groups-which tend to have fewer economic resources-are also less likely to have any debt. Across income groups, borrowing peaks among families above the median. In contrast, by net worth group, debt ownership peaks among families below the median, in the second quartile. Families in the highest three income groups, couples with children, and families headed by a person employed in a managerial or professional position have comparatively high rates of debt ownership.

Debt ownership did not rise uniformly across households between 2004 and 2007. The fraction of families with any debt fell for at least one group within most of the sets of demographic categories shown in table 13. By age group, debt ownership rose 5.5 percentage points for households in the 55-to-64 age group and 6.7 percentage points for those in the 65-to-74 age group, but it fell 8.9 percentage points for families in the oldest age category. Similarly, changes within income and wealth groups ranged from declines of 2 to 3 percentage points to gains of 4 percentage points or more. The percentage of families with debt increased just more than 5 percentage points for nonwhite or Hispanic families as well as for those headed by a self-employed person, whereas the fraction rose more modestly or declined among families in the complementary categories.

The overall median and mean values of outstanding debt for families that had any such debt rose about 11 percent from 2004 to 2007, a slower rate of increase than in the previous three-year period, when the median and mean both rose nearly 34 percent. Median debt tends to rise with income, education, and wealth; the median by age peaks among households headed by a person aged 35 to 44 . The median amount of outstanding debt is also higher for couples, homeowners, and families headed by a person who was self-employed or who was working in a managerial or professional position. Over the recent threeyear period, the median amount of outstanding debt rose for most demographic subgroups. The largest increases in the median amount of debt were for families headed by a person who lacked a high school diploma (47.7 percent) and families headed by a person aged 65 to 74 (45.8 percent); other relatively large increases in the median included those for families living in the South and for families headed by a person who worked in a technical, sales, or service job. The median decreased by the greatest proportion for families in the 75-or-more age group, single families with children, and families living in the Midwest.

\section{Mortgages and Other Borrowing on the Primary Residence}

The share of families with debt secured by a primary residence (hereafter, home-secured debt) continued to trend up, from 47.9 percent in 2004 to 48.7 percent in 2007.43 The increase was driven by the rise in the fraction of homeowners with a mortgage, which rose 1.5 percentage points, to 70.9 percent in 2007.

Families with higher levels of income, education, and wealth are generally more likely to have mortgage debt, as are couples and families headed by a person who is employed in a managerial or professional job or who is self-employed. Across age groups, the rate of borrowing peaks among families in a middle age group and declines sharply among older age groups, a pattern also seen in earlier years. ${ }^{44}$ White non-Hispanic families are more likely to have home-secured debt than are nonwhite or Hispanic families. ${ }^{45}$ Between 2004 and 2007, the prevalence of home-secured debt tended to increase for families with higher levels of income or wealth, and it also rose for families headed by a person who was selfemployed or employed in a technical, sales, or service occupation and for families headed by a person who was aged 55 to 74; the proportion of families with home-secured debt declined most for single-parent families, the oldest age group, and families in the other-not-working category. The measure shifted comparatively little for other demographic groups.

Overall, the median amount of home-secured debt rose 2.6 percent from 2004 to 2007 , and the mean rose 9.4 percent; the median had increased 27.4 per-

43. Home-secured debt consists of first-lien and junior-lien mortgages and home equity lines of credit secured by the primary residence. For purposes of this article, first- and junior-lien mortgages consist only of closed-end loans - that is, loans typically with a one-time extension of credit, a set frequency of repayments, and a required repayment size that may be fixed or vary over time in accordance with a pre-specified agreement or with changes in a given market interest rate. As a type of open-ended credit, home equity lines typically allow credit extensions at the borrower's discretion subject to a prearranged limit and allow repayments at the borrower's discretion subject to a prearranged minimum size and frequency.

44. Of the families that owned a home, the fraction of homeowners with mortgage debt was highest among families in the youngest age group in 2007. For homeowners in the 2004 survey, ownership of home-secured debt peaked among families headed by a person aged 35 to 44 .

45. This pattern reverses, however, when only homeowners are considered; for example, in 2007, 68.9 percent of white non-Hispanic homeowners had a mortgage, compared with 77.7 percent of nonwhite or Hispanic homeowners. 
14. Type of home-secured debt held by homeowners, 1998-2007 surveys Percent

\begin{tabular}{|c|c|c|c|c|}
\hline \multirow{2}{*}{ Type of home-secured debt } & \multicolumn{4}{|c|}{ Homeowners with home-secured debt } \\
\hline & 1998 & 2001 & 2004 & 2007 \\
\hline First-lien mortgage $\ldots$ & 62.2 & 62.6 & 65.2 & 66.1 \\
\hline For home purchase & 36.8 & 35.8 & 28.2 & 30.4 \\
\hline Refinanced & & & & \\
\hline $\begin{array}{l}\text { Extracted equity } \ldots \\
\text { No extracted equity }\end{array}$ & $\begin{array}{r}9.8 \\
156\end{array}$ & 9.7 & 12.9 & 14.3 \\
\hline & & & & \\
\hline Junior-lien mortgage . & 9.4 & 8.5 & 6.1 & 8.5 \\
\hline $\begin{array}{l}\text { For home purchase } \\
\text { Other purpose } . . . .\end{array}$ & $\begin{array}{l}1.0 \\
8.5\end{array}$ & $\begin{array}{l}1.3 \\
7.2\end{array}$ & $\begin{array}{l}1.5 \\
4.7\end{array}$ & $\begin{array}{l}2.1 \\
6.4\end{array}$ \\
\hline Home equity line of credit & 10.6 & 11.2 & 17.8 & 18.4 \\
\hline Currently borrowing .... & 6.7 & 7.1 & 12.4 & 12.4 \\
\hline
\end{tabular}

n.a. Not available (relevant data not collected).

cent over the preceding three years, and the mean had increased 26.9 percent. Changes in the median amount of home-secured debt were mixed across groups. The median fell more than 10 percent for families in the second-lowest income group, families in the top net worth group, and families living in the Northeast. The largest increases in the median value of home-secured debt were for single-parent families and families in the bottom net worth quartile. Both of these groups, but particularly the former, experienced declines in the prevalence of home-secured debt, which suggests that the proportion of smaller home-secured debts among these families fell over the recent period. Other increases in the median were concentrated among the youngest and oldest age groups and among nonwhite or Hispanic families.

The rising values of primary residences over the 2004-07 period outpaced the increases in homesecured debt and, thus, raised the typical amount of home equity held by families with home-secured debt. Median home equity among that group rose from $\$ 76,900$ to $\$ 91,000$ over the period, an 18.3 percent increase (data not shown in the tables). ${ }^{46}$ Among those with such debt, the median ratio of homesecured debt to the value of the primary residence fell 2.7 percentage points, to 53.3 percent in 2007 ; the drop extended a trend in this measure since 1998, when the median ratio was 58.8 percent. Over the recent three-year period, an SCF-based estimate of the aggregate ratio of home-secured debt to home values for all homeowners held steady at 34.9 percent. Nonetheless, at the time of the 2007 SCF interview, 1.0 percent of homeowners had homesecured debt greater than the reported value of their primary residence.

As discussed earlier, home values generally declined after the data collection for the 2007 SCF was completed. Assuming that all else, including home- ownership, stayed constant from the time of the interview until October 2008, the LoanPerformance Home Price Index can be used to approximate the effect of house price declines on home equity. This assumption, together with the house price adjustment, implies that as of October 2008, median home equity for those with mortgage debt was \$71,600 (6.9 percent lower than the 2004 value), and the median ratio of home-secured debt to house values for families with mortgage debt was 58.5 percent. Under this scenario, the aggregate ratio of home-secured debt to house values for homeowners was 39.8 percent in October 2008. ${ }^{47}$

Mortgage interest rates rose slightly, on net, over the 2004-07 period, but they remained low relative to prevailing rates in the 1990s. Comparatively low interest rates, appreciation in house values, changes in mortgage-lending practices, and the deductibility of interest payments on mortgage debt may have provided an incentive for families to borrow against the equity in their home. Such borrowing against home equity may take the form of refinancing an existing first-lien mortgage for more than the outstanding balance, obtaining a junior-lien mortgage, or accessing a home equity line of credit. The survey provides detailed information on all these options for home equity borrowing. The share of homeowners that had a first lien increased 0.9 percentage point, to 66.1 percent in 2007 (table 14). The fraction of homeowners with junior-lien mortgage debt climbed more substantially -2.4 percentage points - to 8.5 percent in 2007. The proportion of homeowners that had a home equity line of credit increased 0.6 percentage

46. Among all homeowners in 2007, median home equity was $\$ 105,000$; in 2004 , it had been $\$ 94,500$.

47. This scenario implies that the adjusted median home equity among all homeowners was \$90,200 in October 2008. 
point, to 18.4 percent in 2007 , but the share of homeowners with an outstanding balance held steady at 12.4 percent; the median amount borrowed against such lines likewise changed little and inched down from $\$ 24,200$ in 2004 to $\$ 24,000$ in 2007 (data not shown in the tables). ${ }^{48}$ Overall, the share of total home-secured debt that was attributable to outstanding balances on home equity lines of credit fell across the 2004 and 2007 surveys (table 14.1).

14.1.

\begin{tabular}{|c|c|c|}
\hline \multirow[b]{2}{*}{ Type of home-secured debt } & \multicolumn{2}{|c|}{ Share of total home-secured debt } \\
\hline & $\begin{array}{c}2007 \\
\text { (percent) }\end{array}$ & $\begin{array}{c}\text { Change, 2004-07 } \\
\text { (percentage points) }\end{array}$ \\
\hline First lien . . & 91.4 & .6 \\
\hline Junior lien $\ldots \ldots \ldots \ldots \ldots$ & 4.0 & 1.0 \\
\hline Home equity line of credit ... & 4.6 & -1.6 \\
\hline
\end{tabular}

In 2007, an increased share of the stock of first liens consisted of either loans for home purchase or loans that had been refinanced and on which the borrower had extracted additional equity at the time of the most recent refinancing (table 14). Among borrowers in the 2007 survey who extracted equity as a part of their most recent refinancing, the median amount extracted was $\$ 28,900$, compared with $\$ 22,000$ in 2004 (data not shown in the tables). The prevalence of both types of junior liens rose over the recent three-year period. In the 2007 survey, the most common use of extracted equity was for home improvement, which accounted for 39.8 percent of outstanding balances attributable to equity extraction on a first lien, a junior lien, or a home equity line of credit.

Families headed by a self-employed person were more likely than families overall to have a home equity line of credit-20.4 percent of self-employed families, compared with 12.6 percent overall in 2007—and to be borrowing against such a line-11.0 percent of self-employed families, compared with 8.5 percent for all families in 2007 (data not shown in the tables). These differences reflect, in part, the relatively higher rates of homeownership among families headed by a self-employed person.

Amid rising house prices between 2004 and 2007, much discussion focused on how families have managed to finance the purchase of a home. One important determinant of the size of the regular payment that families must make to service their mortgages is

48. Of all families, 45.4 percent had a first-lien mortgage in 2007 (45.0 percent in 2004), 5.8 percent had a junior-lien mortgage (4.2 percent in 2004), 12.6 percent had a home equity line of credit (12.3 percent in 2004), and 8.5 percent had a home equity line of credit with an outstanding balance (8.6 percent in 2004). the length of time over which the loan must be repaid. Between 2004 and 2007, the share of fixed-term first-lien mortgages with a term of at least 30 years rose, and the share with a term of 15 years or less declined (table 14.2).

14.2 .

\begin{tabular}{c|c|c}
\hline \multirow{2}{*}{ Mortgage contract length } & \multicolumn{2}{|c}{ First-lien mortgage with a fixed term } \\
\cline { 2 - 3 } & $\begin{array}{c}2007 \\
\text { (percent) }\end{array}$ & $\begin{array}{c}\text { Change, 2004-07 } \\
\text { (percentage points) }\end{array}$ \\
\hline 15 years or shorter $\ldots \ldots \ldots \ldots$ & 25.6 & -7.3 \\
$16-29$ years $\ldots \ldots \ldots \ldots \ldots \ldots$ & 9.4 & -.3 \\
30 years or longer $\ldots \ldots \ldots \ldots \ldots$ & 65.1 & 7.6 \\
\hline
\end{tabular}

Another factor that may affect a borrower's ability to service a loan is the extent to which the payment may change over the life of the loan. Recent declines in house prices and changes in benchmark interest rates have brought particular attention to mortgages with payments that may vary over the life of the loan, including mortgages that do not require the borrower to pay back the entire principal over the contract period of the loan; in such cases, a "balloon payment" of the remaining principal remains at the end of the loan term. From 2004, the fraction of first-lien mortgages on the primary residence that had a potentially variable rate fell 0.8 percentage point, to 14.2 percent in 2007 (data not shown in the tables); over the same period, the share of first-lien mortgages with a balloon payment increased 0.5 percentage point, to 4.6 percent. The level of interest rates is another key determinant of the size of the regular payment that a borrower must make to repay a loan. Between 2004 and 2007, the median interest rate on the stock of outstanding first-lien mortgages on primary residences rose 0.10 percentage point, to 6.00 percent, and the mean interest rate rose 0.13 percentage point, to 6.32 percent.

\section{Borrowing on Other Residential Real Estate}

The overall prevalence of debt owed on residential real estate other than a family's primary residence increased 1.5 percentage points between 2004 and 2007, the largest increase in prevalence of any of the types of debt considered in table 13. The increase reflected not only the rise in the share of families with other residential real estate (discussed earlier) but also a higher rate of borrowing against such properties among families that owned them. In 2004, 32.0 percent of families with other residential real estate owed money on a loan collateralized by the property, and in 2007 this proportion had risen to 40.3 percent. Borrowing on other residential real estate is more common among households with 
higher levels of income, education, or wealth; couples, as well as families headed by a person who was self-employed or who was employed in a managerial or professional position, are also relatively likely to have such debt. These same groups generally experienced the largest increases in the use of such debt.

The median amount of debt on other residential real estate for families having such debt moved up 4.6 percent in 2007 , but the mean amount fell 3.2 percent. These changes are modest compared with the sharp rises between 2001 and 2004 in the median and mean amounts, each of which more than doubled. Changes over the recent three-year period in the median and mean amounts exhibited a mixed pattern of increases and decreases for subgroups of families; shifts in the medians and means for subgroups were generally in the same direction.

\section{Installment Borrowing}

Installment borrowing is about as common as homesecured borrowing. ${ }^{49}$ In 2007, 46.9 percent of families had installment debt, an increase of 0.9 percentage point over the level for 2004. Although the use of installment borrowing has increased in each of the past three surveys, the overall rate of use is comparable with the levels seen in the four surveys from 1989 to 1995 . The use of installment borrowing is broadly distributed across demographic groups, with notably lower use by families in the lowest income group, those in the highest wealth group, childless single families headed by a person aged 55 or older, families headed by a retired person, and families headed by a person aged 65 or older. By comparison, the median amount of outstanding installment debt varies more clearly across many groups: That amount tends to rise with income, education, and occupational status, and it falls with age. The median amount of installment debt is fairly comparable for families with net worth below the 90th percentile and is sharply higher for families in the top net worth group.

Installment borrowing is used for a wide variety of purposes. In 2007, 51.7 percent of such borrowing was related to the purchase of a vehicle, and 33.2 percent of outstanding installment debt was owed for educational purposes. In general, balances on vehicle loans account for a disproportionate share of installment debt for those families headed by a person with at most a high school degree; vehicle debt constitutes a relatively low proportion of total installment debt

49. The term "installment borrowing" in this article describes closed-end consumer loans - that is, loans that typically have fixed payments and a fixed term. Examples are automobile loans, student loans, and loans for furniture, appliances, and other durable goods. for younger families and families in the lowest wealth category shown (table 15); the shares of installment debt attributable to education loans decline with age and wealth, and-as might be expected - the share rises sharply with education. ${ }^{50}$

From 2004 to 2007, the median amount owed on installment loans rose 2.4 percent, and the mean rose 1.4 percent. Changes in the median within demographic categories include both increases and decreases. The largest gains occurred among families in the second net worth quartile and families headed by a person who was retired or otherwise not working, while the sharpest declines occurred among families headed by a person aged 55 to 64 and childless single families headed by a person aged 55 or older.

\section{Credit Card Balances and Other Lines of Credit}

As with installment borrowing, the carrying of credit card balances is widespread but considerably less common among the highest and lowest income groups, the highest wealth group, and families headed by a person who is aged 65 or older or who is retired. ${ }^{51}$ The proportion of families carrying a balance, 46.1 percent in 2007, was barely changed from 2004. Underlying this stability in the share of all families carrying a balance were larger shifts for many demographic groups, with increases and decreases of 3 percentage points or more for many of the groups.

Overall, the median balance for those carrying a balance rose 25.0 percent, to $\$ 3,000$; the mean rose 30.4 percent, to $\$ 7,300$. These increases followed slower changes over the preceding three years, when the median increased 9.1 percent and the mean climbed 16.7 percent (data not shown in the tables). Over the recent period, the median balance rose strongly for most demographic groups, particularly for higher-income families, childless couples, and families headed by a person who was aged 55 to 64 or who was self-employed. However, the median balance fell roughly 30 percent for the oldest age group, younger childless single families, and families headed

50. For an expanded version of table 13, including the categories of installment loans given in table 15, see www.federalreserve.gov/pubs/ oss/oss2/2007/scf2007home.html.

51. In this article, credit card balances consist of balances on bank-type cards (such as Visa, MasterCard, and Discover as well as Optima and other American Express cards that routinely allow carrying a balance), store cards or charge accounts, gasoline company cards, so-called travel and entertainment cards (such as American Express cards that do not routinely allow carrying a balance and Diners Club), other credit cards, and revolving store accounts that are not tied to a credit card. Balances exclude purchases made after the most recent bill was paid. 
15. Value of installment debt distributed by type of installment debt by selected characteristics of families with installment debt, 2004 and 2007 surveys

Percent

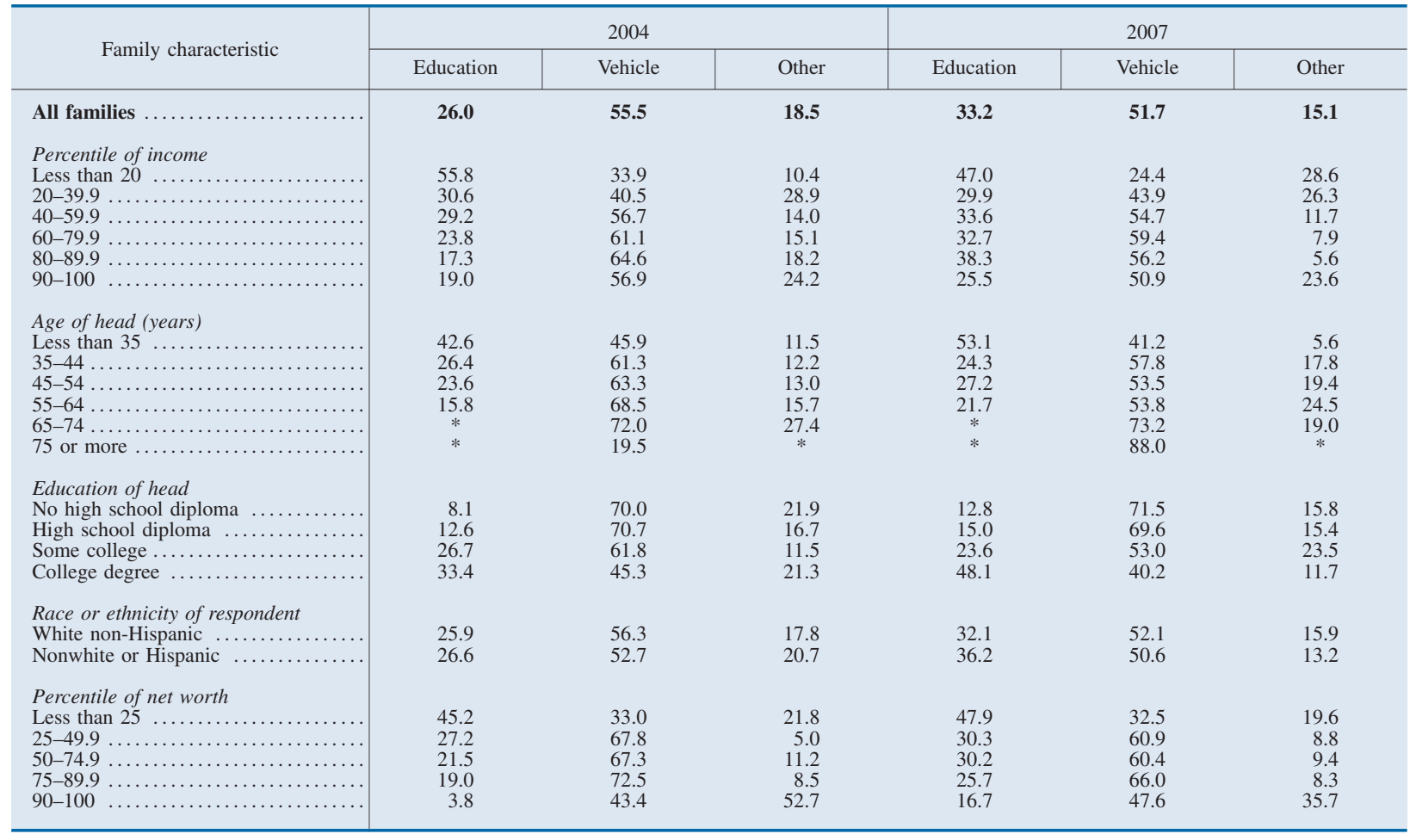

Note: See note to table 1.

* Ten or fewer observations.

by a person who was neither working nor retired; median balances declined more modestly for selected other groups.

Many families with credit cards do not carry a balance. ${ }^{52}$ Of the 73.0 percent of families with credit cards in 2007, only 60.3 percent had a balance at the time of the interview; in 2004, 74.9 percent had cards, and 58.0 percent of these families had an outstanding balance on them (data not shown in the tables). The proportion of cardholders who had bank-type cards increased over this three-year period, as did the proportion with miscellaneous other credit cards, while the share of cardholders having gasoline or travel and entertainment card types declined considerably (table 15.1). These declines probably reflect, at least in part, a rise during the period in the issuance of bank-type cards under the brand names of stores and

52. The remaining discussion of credit cards excludes revolving store accounts that are not tied to a credit card. In 2007, 5.4 percent (5.9 percent in 2004) of families had such an account, the median outstanding balance for families that had a balance was $\$ 700$ ( $\$ 790$ in 2004), and the total of such balances accounted for 4.4 percent (4.3 percent in 2004) of the total of balances on credit cards and such store accounts (data not shown in the tables).
15.1.

\begin{tabular}{|c|c|c|}
\hline \multirow[b]{2}{*}{ Type of credit card } & \multicolumn{2}{|c|}{ Families with credit cards } \\
\hline & $\begin{array}{c}2007 \\
\text { (percent) }\end{array}$ & $\begin{array}{l}\text { Change, 2004-07 } \\
\text { (percentage points) }\end{array}$ \\
\hline Bank & 96.1 & .7 \\
\hline Store ... & 56.7 & -1.7 \\
\hline Gasoline .................... & 11.9 & -5.4 \\
\hline Travel and entertainment .... & 7.4 & -2.6 \\
\hline Miscellaneous ................ & 3.7 & 1.1 \\
\hline
\end{tabular}

gasoline companies and in the issuance of new types of American Express cards that routinely allow carrying a balance.

Bank-type cards are the most widely held type of card and thus hold particular importance. Indeed, balances on such cards accounted for 87.1 percent of outstanding credit card balances in 2007, up from 84.9 percent in 2004 (data not shown in the tables). The proportion of holders of bank-type cards who had a balance went up 2.1 percentage points, to 58.3 percent; the proportion of holders of bank-type cards who reported that they usually pay their balances in full retreated a bit, from 55.7 percent in 2004 to 55.3 percent in 2007. Over the recent three-year period, the median outstanding charges for the month preceding the interview on all bank-type cards held 
by the family fell from $\$ 280$ in 2004 to $\$ 250$ in 2007. For families having any bank-type cards, the median number of such cards remained at 2 ; the median credit limit on all such cards rose 21.4 percent, to $\$ 18,000$, and the median interest rate on the card with the largest balance (or on the newest card, if no outstanding balances existed) rose 1.0 percentage point, to 12.5 percent.

Only 3.8 percent of families had an established line of credit other than a home equity line in 2007 (data not shown in the tables). ${ }^{53}$ Even fewer families1.7 percent - had a balance on such a line, an increase of 0.1 percentage point since 2004 and only 0.2 percentage point since 2001 . The median amount outstanding on these lines climbed 15.2 percent between the most recent surveys, while the mean fell 38.3 percent.

Borrowing on other lines of credit was more common among households headed by a person who was self-employed, a pattern that is apparent in earlier SCF surveys; a similar pattern also holds when the analysis considers all available lines, not just those against which families carried a balance.

\section{Other Debt}

From 2004 to 2007, the proportion of families that owed money on other types of debts decreased 0.8 percentage point, to 6.8 percent. ${ }^{54}$ The ownership of each underlying type of such debt also declined (table 15.2).

15.2 .

\begin{tabular}{l|c|c}
\hline \multirow{2}{*}{ Type of other debt } & \multicolumn{2}{|c}{ All families } \\
\cline { 2 - 3 } & $\begin{array}{c}2007 \\
\text { (percent) }\end{array}$ & $\begin{array}{c}\text { Change, 2004-07 } \\
\text { (percentage points) }\end{array}$ \\
\hline Cash value life insurance & & \\
loans ................ & .9 & -0.7 \\
Pension account loans ...... & 3.4 & -0.1 \\
Margin account loans ....... & .5 & $\dagger$ \\
Other miscellaneous loans ... & 2.4 & -.3 \\
\hline & & \\
\hline Less than 0.05 percent.
\end{tabular}

Rates of use of other debt were noticeably lower for families in the bottom income group as well as for families headed by a person who is 75 years of age or older or who is retired and for families in the next-tohighest net worth group. The highest rate of other debt ownership was for single families with children. The prevalence of such debt fell for families with

53. In this article, borrowing on lines of credit excludes borrowing on credit cards.

54. The "other debt" category comprises loans on cash value life insurance policies, loans against pension accounts, borrowing on margin accounts, and a miscellaneous category largely comprising personal loans not explicitly categorized elsewhere. higher levels of income, education, or net worth; the rate of use rose for the age groups between 45 and 74 and for all occupation categories except the managerial-or-professional group.

The median amount owed by families with this type of debt rose 13.6 percent, to $\$ 5,000$, between 2004 and 2007; over the same period, the mean fell 17.1 percent. In 2007, 36.6 percent of the total amount of this type of debt was attributable to margin loans (50.4 percent in 2004), 21.3 percent to loans against a pension from a current job of the family head or that person's spouse or partner (21.2 percent in 2004), 12.1 percent to loans against cash value life insurance policies (9.8 percent in 2004), and the remaining 30.0 percent to miscellaneous loans (18.7 percent in 2004) (data not shown in the tables).

In 2007, the SCF collected information for the first time on whether a family member had taken out a loan in the past year that was supposed to be repaid in full out of that person's next paycheck. ${ }^{55}$ Overall, 2.4 percent of families reported having taken out a so-called payday loan. The fraction of families that had taken out a payday loan declined with age, falling from 4.9 percent of families headed by a person younger than age 35 to essentially 0 percent for families headed by a person aged 65 or older (data not shown in the tables). Across income groups, the share of families that reported such a loan was between 3.5 percent and 4.0 percent for the bottom three quintiles, but families in the top two quintiles reported virtually no use of this type of short-term loan. Similarly, 5.8 percent of families in the bottom net worth quartile reported having taken out a payday loan, while 3.7 percent of families in the second quartile and virtually no families with net worth above the median reported having done so.

The data indicate that families tend to take out payday loans to finance immediate expenses. The most common reason given for choosing a payday loan for families that had taken out such a loan was "emergencies" and similar urgent needs or a lack of other options (35.9 percent). ${ }^{56}$ Roughly equal shares of families cited convenience in obtaining the loan (21.0 percent) or the need to pay for living expenses, including food, gas, vehicle expenses, medical payments, utility costs, or rent (20.6 percent). A smaller fraction, 10.8 percent, of these families reported a need to pay other bills and loans. The remaining

55. The family may or may not have had such a loan outstanding at the time of the interview.

56. This discussion considers the primary reasons given by families when asked why they chose this type of loan. Families could provide up to two reasons, but 92.0 percent of those who had taken out a payday loan in the past year provided only one. 
12.6 percent of families with a payday loan in the past year cited other needs, including "Christmas" or the need to "help family."

\section{Reasons for Borrowing}

The SCF provides information on the reasons that families borrow money (table 16). One subtle problem with the use of these data is that, even though money is borrowed for a particular purpose, it may be employed to offset some other use of funds. For example, a family may have sufficient funds to purchase a home without using a mortgage but may instead choose to finance the purchase to free existing funds for another purpose. Thus, trends in the data can only suggest the underlying use of funds by families.

Although the survey information on use is substantial, it is not exhaustive. Most important, in the case of credit cards, it was deemed impractical to ask about the purposes of borrowing, which might well be heterogeneous for individual families. For the analysis here, all credit card debt is included in the category "goods and services." The surveys before 2004 lack information on the use of funds borrowed through a first-lien mortgage; therefore, for purposes of this calculation, all funds owed on a first-lien mortgage on a primary residence are assumed to have been used for the purchase of the home, even when the homeowner had refinanced the mortgage and extracted equity for another purpose.

The great majority of family debt is attributable to the purchase of a primary residence; however, from 2004 to 2007, the share of debt for this purpose declined 0.7 percentage point after a similar decline in the 2004 survey. Looking more broadly at debt for residential real estate, the drop in debt for home purchase was more than offset by both an increase in balances owed on residential real estate other than the primary residence - the second-largest share of debtand a slight rise in balances owed for improvements

16. Amount of debt of all families, distributed by purpose of debt, 1998-2007 surveys

Percent

\begin{tabular}{|c|c|c|c|c|}
\hline Purpose of debt & 1998 & 2001 & 2004 & 2007 \\
\hline \multicolumn{5}{|l|}{ Primary residence } \\
\hline Purchase .............. & 67.9 & 70.9 & 70.2 & 69.5 \\
\hline Improvement .......... & 2.1 & 2.0 & 1.9 & 2.3 \\
\hline Other residential property ....... & 7.8 & 6.5 & 9.5 & 10.8 \\
\hline Investments excluding real estate & 3.3 & 2.8 & 2.2 & 1.6 \\
\hline Vehicles $\ldots \ldots \ldots \ldots \ldots \ldots \ldots$ & 7.6 & 7.8 & 6.7 & 5.5 \\
\hline Goods and services & 6.3 & 5.8 & 6.0 & 6.2 \\
\hline Education ................ & 3.5 & 3.1 & 3.0 & 3.6 \\
\hline Other $\ldots \ldots \ldots \ldots \ldots \ldots$ & 1.5 & 1.1 & .6 & .5 \\
\hline Total & 100 & 100 & 100 & 100 \\
\hline
\end{tabular}

Note: See note to table 8.
17. Amount of debt of all families, distributed by type of lending institution, 1998-2007 surveys

Percent

\begin{tabular}{|c|c|c|c|c|}
\hline Type of institution & 1998 & 2001 & 2004 & 2007 \\
\hline Commercial bank & 32.8 & 34.1 & 35.1 & 37.3 \\
\hline Thrift institution ${ }^{1}$ & 9.7 & 6.1 & 7.3 & 4.2 \\
\hline Credit union $\ldots \ldots \ldots \ldots$ & 4.3 & 5.5 & 3.6 & 4.2 \\
\hline Finance or loan company & 4.1 & 4.3 & 4.1 & 3.4 \\
\hline Brokerage $\ldots \ldots \ldots \ldots \ldots \ldots$ & 3.8 & 3.1 & 2.5 & 1.6 \\
\hline Mortgage or real estate lender & 35.6 & 38.0 & 39.4 & 41.6 \\
\hline Individual lender $\ldots \ldots \ldots \ldots \ldots$ & 3.3 & 2.0 & 1.7 & 1.4 \\
\hline Other nonfinancial & 1.3 & 1.4 & 2.0 & 2.0 \\
\hline Government ........... & .6 & 1.1 & .7 & .4 \\
\hline Credit card issuer .......... & 3.9 & 3.7 & 3.0 & 3.6 \\
\hline Pension $\ldots \ldots \ldots \ldots \ldots \ldots$ & .4 & .3 & .3 & .2 \\
\hline Other $\ldots \ldots \ldots \ldots \ldots \ldots$ & 3 & .5 & .2 & .2 \\
\hline Total & 100 & 100 & 100 & 100 \\
\hline
\end{tabular}

Note: See note to table 1.

1. Savings and loan association or savings bank.

on the primary residence. In 2007, the fraction of debt owed for goods and services exceeded the share of borrowing for vehicles for the first time in any SCF survey since 1989 , largely because of a decline in the share for vehicles between 2004 and 2007. The majority of the debt in the goods and services category, 56.5 percent, was outstanding balances on credit cards..$^{57}$

\section{Choice of Lenders}

The survey provides information on the types of lenders to which families owe money at the time of the interview (table 17). Over the past decade, regulatory changes and other shifts have contributed to consolidation of financial institutions; at the same time, consumers have witnessed a continuing proliferation of similarly named subsidiaries of large financial institutions, which may offer a variety of possibly overlapping financial services. As a result, families in the SCF appear to have had difficulty in accurately classifying the institutional type of lender holding their loans. A parent company may, for example, offer installment loans through both a subsidiary commercial bank and a subsidiary finance company with similar names. Thus, the proportions shown in the table are only indicative, and small differences across categories or years should be interpreted with particular caution.

The share of total debt reportedly owed to thrift institutions (savings and loan associations and sav-

57. The surveys beginning with 2004 contain information on the use of funds obtained from refinancing a first-lien mortgage. If this information for 2007 is used to classify outstanding debt by purposes, the shares of debt were, for home purchase, 65.6 percent; for home improvements, 3.9 percent; for other residential real estate, 11.1 percent; for investments other than real estate, 1.9 percent; for vehicles, 5.7 percent; for goods and services, 7.7 percent; for education, 3.6 percent; and for other unclassified purposes, 0.5 percent. 
ings banks) fell 3.1 percentage points between 2004 and 2007. The estimated shares held by finance and loan companies or brokerages declined 0.7 and 0.9 percentage point, respectively. The largest increases over the period were the reported rise of 2.2 percentage points in the shares of debt owed to a commercial bank and to mortgage or real estate lenders, followed by gains of 0.6 percentage point for both credit unions and credit card issuers.

In some cases, loans may have been held at the time of the interviews by institutions other than the ones that originally made the loans. This fact might likewise make determining the type of financial institution that holds such debt more difficult. Resale of loans is particularly important for mortgage debt. According to the 2007 survey, 39.5 percent of the first-lien mortgages on primary residences were held by lenders other than the ones that made the original loans, a figure 2.0 percentage points lower than in $2004 .{ }^{58}$ In dollar-weighted terms, the results are similar; mortgages with non-originating lenders accounted for 40.3 percent of the outstanding balances on first-lien mortgages for primary residences in 2007 and 43.3 percent in 2004 (data not shown in the tables).

\section{Credit Market Experiences}

The SCF also collects some information on families' recent credit market experiences. Specifically, the survey asks whether the family had applied for any type of credit in the past five years and, if so, whether any application was either turned down or granted for a lesser amount than the amount initially requested. Families that gave such responses were asked the reason given for the decision. The survey also asks whether, at any time in the past five years, the family ever considered applying for credit but then decided not to apply because of a belief that the application would be rejected. Such families were asked the reason they believed they would have been turned down.

In 2007, 66.3 percent of families had applied for credit at some point in the preceding five years (68.7 percent in 2004). Of these families, 29.7 percent had at least once been either turned down for credit or approved for less credit than they had applied for in the past five years (30.4 percent in 2004). Of all

58. Mortgages and other loans may also be serviced by an institution other than the current lender, and some respondents may mistakenly report their loan as having been sold even though it is simply being serviced by an institution other than the current lender. Because a loan can also be sold without changing the servicer, some borrowers may mistakenly report that their loan has not been sold. families, 15.3 percent had considered applying but subsequently did not do so because they thought the application would be denied (15.8 percent in 2004). The most common reasons reported for either having been denied credit or having not applied for credit were reasons related to the borrower's credit characteristics, such as the lack of a credit history, previous performance on a loan or account from another institution, and the amount of debt held by the borrower (table 17.1). ${ }^{59}$

17.1.

\begin{tabular}{|c|c|c|}
\hline $\begin{array}{l}\text { Reason turned down } \\
\text { or did not apply }\end{array}$ & $\begin{array}{l}\text { Families who applied } \\
\text { for credit and were } \\
\text { turned down or } \\
\text { received less credit } \\
\text { than the amount } \\
\text { requested (percent) }\end{array}$ & $\begin{array}{l}\text { Families who did } \\
\text { not apply for credit } \\
\text { because they expected } \\
\text { to be turned down } \\
\text { (percent) }\end{array}$ \\
\hline Personal characteristics & 1.8 & 3.9 \\
\hline Credit characteristics .. & 59.9 & 67.7 \\
\hline Financial characteristics .. & 29.4 & 22.9 \\
\hline \multirow{2}{*}{$\begin{array}{l}\text { Miscellaneous, including } \\
\text { no reason given ........ }\end{array}$} & & \\
\hline & 8.8 & 5.5 \\
\hline
\end{tabular}

\section{Debt Burden}

The ability of individual families to service their loans is a function of two factors: the level of their loan payments and the income and assets they have available to meet those payments. In planning their borrowing, families make assumptions about their future ability to repay their loans. Problems may occur when events turn out to be contrary to those assumptions. If such misjudgments are sufficiently large and prevalent, a broad pattern of default, restraint in spending, and financial distress in the wider economy might ensue.

The Federal Reserve staff has constructed an aggregate-level debt service ratio, defined as an estimate of total scheduled loan payments (interest plus minimum repayments of principal) for all households, divided by disposable personal income. From the third quarter of 2004 to the same period in 2007, the aggregate-level measure stepped up 0.74 percentage point, to 14.39 percent. ${ }^{60}$

59. Personal characteristics include responses related to family background or size, marital status, sex, or age; credit characteristics include responses related to the need to have a checking or savings account, lack of a credit history, credit reports from a credit rating agency or from other institutions, or the level of outstanding debt and insufficient credit references; financial characteristics include responses related to previous difficulty getting credit, more "strict" lending requirements of the institution, an error in processing the application, or credit problems of an ex-spouse.

60. Data on this measure, the "debt service ratio," and a description of the series are available at www.federalreserve.gov/releases/ housedebt/default.htm. See Karen Dynan, Kathleen Johnson, and 
18. Ratio of debt payments to family income (aggregate and median), share of debtor families with ratio greater than 40 percent, and share of debtors with any payment 60 days or more past due, 1998-2007 surveys

Percent

\begin{tabular}{|c|c|c|c|c|c|c|c|c|c|c|c|c|c|c|c|c|}
\hline \multirow{2}{*}{ Family characteristic } & \multicolumn{4}{|c|}{ Aggregate } & \multicolumn{4}{|c|}{ Median for debtors } & \multicolumn{4}{|c|}{$\begin{array}{l}\text { Debtors with ratio greater } \\
\text { than } 40 \text { percent }\end{array}$} & \multicolumn{4}{|c|}{$\begin{array}{l}\text { Debtors with any payment } \\
\text { past due } 60 \text { days or more }\end{array}$} \\
\hline & 1998 & 2001 & 2004 & 2007 & 1998 & 2001 & 2004 & 2007 & 1998 & 2001 & 2004 & 2007 & 1998 & 2001 & 2004 & 2007 \\
\hline All families & 14.9 & 12.9 & 14.4 & 14.5 & 17.9 & 16.7 & 18.0 & 18.6 & 13.6 & 11.8 & 12.2 & 14.7 & 8.1 & 7.0 & 8.9 & 7.1 \\
\hline Percentile of income & & & & & & & & & & & & & & & & \\
\hline Less than $20 \ldots \ldots$. & 18.8 & 16.1 & 18.2 & 17.6 & 18.6 & 19.2 & 19.7 & 19.0 & 29.8 & 29.3 & 26.8 & 26.9 & 13.0 & 13.4 & 15.9 & 15.1 \\
\hline $20-39.9 \ldots \ldots \ldots \ldots$ & 16.6 & 15.8 & 16.6 & 17.2 & 17.5 & 16.7 & 17.4 & 17.0 & 18.3 & 16.6 & 18.5 & 19.5 & 12.4 & 11.7 & 13.8 & 11.5 \\
\hline $40-59.9$ & 18.7 & 17.1 & 19.4 & 19.8 & 19.4 & 17.6 & 19.5 & 20.3 & 15.9 & 12.3 & 13.7 & 14.5 & 10.0 & 7.9 & 10.4 & 8.3 \\
\hline $60-79.9$ & 19.1 & 16.8 & 18.5 & 21.7 & 19.5 & 18.1 & 20.6 & 21.9 & 9.8 & 6.5 & 7.1 & 12.7 & 5.9 & 4.0 & 7.1 & 4.1 \\
\hline $80-89.9$ & 16.8 & 17.0 & 17.3 & 19.7 & 17.8 & 17.2 & 18.1 & 19.3 & 3.5 & 3.5 & 2.4 & 8.1 & 3.9 & 2.6 & 2.3 & 2.1 \\
\hline $90-100$ & 10.3 & 8.1 & 9.3 & 8.4 & 13.7 & 11.2 & 12.7 & 12.5 & 2.8 & 2.0 & 1.8 & 3.8 & 1.6 & 1.3 & .3 & .2 \\
\hline \multicolumn{17}{|l|}{ Age of head (years) } \\
\hline Less than $35 \ldots \ldots$ & 17.2 & 17.2 & 17.8 & 19.7 & 16.9 & 17.7 & 18.0 & 17.5 & 12.9 & 12.0 & 12.8 & 15.1 & 11.1 & 11.9 & 13.7 & 9.4 \\
\hline $35-44 \ldots \ldots \ldots$ & 17.7 & 15.1 & 18.2 & 18.5 & 20.0 & 17.8 & 20.6 & 20.3 & 12.5 & 10.1 & 12.5 & 12.7 & 8.4 & 5.9 & 11.7 & 8.6 \\
\hline $45-54$ & 16.4 & 12.8 & 15.3 & 14.9 & 17.9 & 17.4 & 18.4 & 19.3 & 12.8 & 11.6 & 13.1 & 16.0 & 7.4 & 6.2 & 7.6 & 7.3 \\
\hline $55-64$ & 13.4 & 10.9 & 11.5 & 12.5 & 17.6 & 14.3 & 15.7 & 17.5 & 14.0 & 12.3 & 10.2 & 14.5 & 7.5 & 7.1 & 4.2 & 4.9 \\
\hline $65-74 \ldots \ldots$ & 8.8 & 9.2 & 8.7 & 9.6 & 13.2 & 16.0 & 15.6 & 17.9 & 18.1 & 14.7 & 11.6 & 15.6 & 3.1 & 1.5 & 3.4 & 4.4 \\
\hline 75 or more & 4.1 & 3.9 & 7.1 & 4.4 & 8.1 & 8.0 & 12.8 & 13.0 & 21.4 & 14.6 & 10.7 & 13.9 & 1.1 & .8 & 3.9 & 1.0 \\
\hline \multicolumn{17}{|l|}{ Percentile of net worth } \\
\hline Less than $25 \ldots \ldots \ldots$ & 15.0 & 13.4 & 13.0 & 15.0 & 13.6 & 11.5 & 13.0 & 12.1 & 13.1 & 11.6 & 10.5 & 10.4 & 16.3 & 17.7 & 22.9 & 16.8 \\
\hline $25-49.9 \ldots$ & 20.1 & 18.1 & 19.5 & 22.4 & 20.2 & 20.1 & 21.2 & 23.4 & 15.9 & 14.2 & 15.8 & 19.3 & 9.8 & 7.1 & 11.0 & 7.7 \\
\hline $50-74.9$ & 18.3 & 16.7 & 20.6 & 20.3 & 20.2 & 18.3 & 21.4 & 21.5 & 13.0 & 11.2 & 12.8 & 15.9 & 5.5 & 3.6 & 3.2 & 4.2 \\
\hline $75-89.9$ & 14.8 & 15.4 & 15.1 & 17.0 & 17.8 & 16.9 & 17.8 & 18.2 & 12.3 & 10.6 & 9.6 & 13.0 & 1.0 & .7 & 1.1 & 1.2 \\
\hline $90-100 \ldots \ldots$ & 10.2 & 7.4 & 8.5 & 8.0 & 14.1 & 11.2 & 12.6 & 12.6 & 12.2 & 8.5 & 7.6 & 11.1 & 2.4 & .3 & .1 & .7 \\
\hline \multicolumn{17}{|l|}{ Housing status } \\
\hline Owner...$\ldots \ldots$ & 16.3 & 13.9 & 15.6 & 15.6 & 21.2 & 20.0 & 21.5 & 22.8 & 16.5 & 14.7 & 14.9 & 18.0 & 6.1 & 4.3 & 5.6 & 4.8 \\
\hline Renter or other & 8.2 & 7.4 & 7.2 & 7.9 & 8.5 & 8.3 & 8.1 & 8.2 & 6.5 & 4.2 & 4.3 & 5.4 & 12.9 & 14.0 & 18.6 & 13.5 \\
\hline
\end{tabular}

Note: The aggregate measure is the ratio of total debt payments to total income for all families. The median is the median of the distribution of ratios calculated for individual families with debt. Also see note to table 1 .

The survey data for individual families may be used to construct a similar estimate of debt burden for families overall as well as for various demographic groups (table 18). ${ }^{61}$ The SCF-based estimate is the ratio of total debt payments for all families to total

Karen Pence (2003), "Recent Changes to a Measure of U.S. Household Debt Service," Federal Reserve Bulletin, vol. 89 (October), pp. 417-26.

61. The survey measure of payments relative to income may differ from the aggregate-level measure for several reasons. First, the debt payments included in each measure are different. The aggregate-level measure includes only debts originated by depositories, finance companies, and other financial institutions, whereas the survey includes, in principle, debts from all sources.

Second, the aggregate-level measure uses an estimate of disposable personal income from the national income and product accounts for the period concurrent with the estimated payments as the denominator of the ratio, whereas the survey measure uses total before-tax income reported by survey families for the preceding year; the differences in these two income measures are complex.

Third, the payments in the aggregate-level measure are estimated using a formula that entails complex assumptions about minimum payments and the distribution of loan terms at any given time; the survey measure of payments is directly asked of the survey respondents but may also include payments of taxes and insurance on real estate loans.

Fourth, because the survey measures of payments and income are based on the responses of a sample of respondents, they may be affected both by sampling error and by various types of response errors. As mentioned earlier in this article, the survey income measure tracks the most comparable measure of income in the Census Bureau's Current Population Survey. family income of all families. ${ }^{62}$ From 2004 to 2007 , the SCF-based estimate rose, albeit by less than the aggregate-level measure, increasing 0.1 percentage point, to 14.5 percent. In the previous three-year period, the SCF measure had increased at a faster pace than the aggregate-level measure; between 2001 and 2004, the aggregate estimate of the debt-burden ratio rose 1.4 percentage points, and the SCF-based measure increased 1.5 percentage points. If total payments and incomes are computed from the survey data using only families with debt payments, the results for the recent period show a slightly larger increase, from 17.7 percent in 2004 to 18.0 percent in 2007; if the ratio is computed using only families with home-secured debt, the data show a rise from 20.2 percent in 2004 to 20.5 percent in 2007 (data not shown in the tables). The SCF-based estimate of the aggregate debt-burden ratio increased for most demographic groups over the recent three-year period.

62. The definition of debt payments in the SCF does not include payments on leases or rental payments. The survey collects information on vehicle lease payments and rent on primary residences, and, thus, in principle a broader measure of debt payments could be constructed, one that would be similar to the "financial obligations ratio" estimated by the Federal Reserve staff. 
The ability to look at the distribution of payments relative to income at the level of families potentially offers insights that are not available from any of the aggregate-level figures. In particular, the survey allows a detailed look at the spectrum of payments relative to income across all families with debts. Over the recent period, the median of the ratios for individual families that had any debt rose 0.6 percentage point, to 18.6 percent in 2007 , a gain that extends a series of increases in this measure since 1989 that were interrupted only by a decline between 1998 and 2001. The median ratio of debt payments to income also rose at least slightly in the recent period for most demographic groups shown; the median fell for families with wealth in the lowest quartile, for families with income in the two lowest quintiles or the highest decile, and for families headed by a person younger than 45.63

A limitation of the median ratio is that it may not be indicative of distress because it reflects the situation of only a typical family. Unless errors of judgment by both families and lenders are pervasive, one would not expect to see signs of financial distress at the median. Thus, a more compelling indicator of distress is the proportion of families with unusually large total payments relative to their incomes. From 2004 to 2007, the proportion of debtors with payments exceeding 40 percent of their incomes rose 2.5 percentage points, to 14.7 percent; in the preceding three years, the proportion had increased 0.4 percentage point. The increase was shared by all demographic groups except families in the bottom net worth group, for which the share edged back 0.1 percentage point, to 10.4 percent; in contrast, this fraction increased between 3.0 and 3.5 percentage points for each of the other net worth groups. Compared with the increases for lower income groups, the share of families with income between the 60th and 90th percentiles who had a relatively high ratio of debt payment to income rose especially sharply. ${ }^{64}$

Fluctuations in a family's income away from its usual level can have substantial effect on the family's payment-to-income ratio. If the ratio is defined in terms of families' reported usual incomes, the fraction of families with a ratio exceeding 40 percent falls to 13.6 percent. This 1.1 percentage point difference reflects two facts: (1) 2.5 percent of families with debt

63. The median of the ratio for families with home-secured debt in 2007 was 25.1 percent, up from 24.2 percent in 2001 (data not shown in the tables).

64. Of families with home-secured debt, the proportion that had total payments of more than 40 percent of their income was 20.1 percent in 2007, a level 3.0 percentage points higher than that in 2004 (data not shown in the tables). had relatively high payment-to-income ratios based on the previous year's income but would not have if income had been at its usual level, and (2) 1.4 percent of families with debt had debt payments less than or equal to 40 percent of last year's income but would have had a ratio above 40 percent if income had been at its usual level. Families may draw on assets as well as income to meet debt payments. For all families with debt, 57.7 percent had transaction account balances equal to at least three months of debt payments. For families with payment-to-income ratios above 40 percent, however, this share falls to 25.9 percent.

Other commonly used indicators of debt-repayment problems are aggregate delinquency rates-that is, the percentage of delinquent accounts or the percentage of total balances on which payments are late. Both account-based and dollar-weighted aggregate measures indicate that delinquencies on mortgages rose, on net, from the third quarter of 2004 to the third quarter of 2007, but they began to rise more sharply thereafter. Over the 2004-07 period, the percentage of delinquent automobile loans declined, while a corresponding dollar-weighted measure rose; the fraction of delinquent loans leveled off in 2008, while the dollar-weighted measure continued to rise. On net, a dollar-weighted delinquency measure for other closedend loans was unchanged from the third quarter of 2004 to the third quarter of 2007, while the percentage of delinquent loans rose; over the following year, both measures rose. Delinquency measures for credit cards also differed by whether the measure was based on dollar volume or delinquent accounts, but all pointed to comparatively small changes between the third quarter of 2004 and the third quarter of 2007; over the following four quarters, all of these measures showed clear increases. ${ }^{65}$

A related measure is collected in the SCF. Families that have any debt at the time of their interview are asked whether they have been behind in any of their loan payments in the preceding year. This measure differs conceptually from the aggregate delinquency rates in that the survey counts multiple occasions of late payments as one, counts families instead of balances or accounts, and includes all types of loans; because it counts individual families, not their balances, it is closer in spirit to aggregate measures based on the numbers of delinquent accounts than to those based on the amounts of delinquent balances. The survey shows a decrease from 8.9 percent in 2004 to 7.1 percent in 2007 in the proportion of

65. The most commonly used such measures are from the Consolidated Reports of Condition and Income (Call Report), the American Bankers Association, and Moody's Investors Service. 
debtors who were 60 or more days late with their payments on any of their loans in the preceding year. This measure fell for families in each of the income groups but particularly for families in the middle 60 percent of the income distribution; the percentage declined for families with net worth below the median, and it rose for families with higher levels of net worth. 66 The share of families with debt who were at least 60 days late on a payment during the preceding year rose for families headed by a person aged 55 to 74 and fell for both homeowners and, more substantially, renters. For families with a payment-toincome ratio of 40 percent or more, 13.9 percent missed a debt payment by 60 days or more; by comparison, 6.0 percent of debtor families with lower ratios had fallen behind in debt repayment.

\section{SUMMARY}

Data from the 2004 and 2007 SCF show that median income barely changed, while mean income rose substantially, an indication that income gains were much greater for families in the uppermost part of the distribution. Although overall both median and mean net worth increased strongly over this period-17.7 percent and 13.0 percent, respectively-these measures declined for families at the bottom of the wealth distribution. The preceding three years had seen only small changes in median and mean income and in median net worth but a sizable gain in mean net worth.

Although the median and mean of families' holdings of financial assets increased overall from 2004 to 2007, financial assets declined as a share of total assets, continuing an earlier trend. The offsetting expansion in the share of nonfinancial assets was most strongly driven by greater holdings of private business equity and, to a lesser degree, of residential real estate other than a primary residence. The homeownership rate, which had risen noticeably between the 2001 and 2004 surveys, turned down slightly. Unrealized capital gains were an important part of the increase in assets; in 2007, 35.8 percent of total assets was attributable to unrealized capital gains, and those gains were most concentrated in holdings of real estate and private business equity. In 2004, unrealized gains accounted for 30.7 percent of assets.

Debt and assets rose in about equal proportions over the recent three-year period. Thus, overall indebtedness as a share of assets was little changed.

66. For families with home-secured debt, the result is very similar to that for homeowners overall. The proportion with payments late 60 days or more in 2007 was 4.8 percent after rising to an estimated 5.7 percent in 2004 (data not shown in the tables).
Home-secured debt fell slightly as a share of total family debt, but in 2007 it remained by far the largest component of family debt. The share of borrowing for residential real estate other than the primary residence increased appreciably. The percentage of families using credit cards for borrowing changed only slightly over the period, but the median balance on their accounts rose 25.0 percent, and the mean rose 30.4 percent.

Despite a moderate rise in typical consumer loan interest rates from 2004 to 2007 , the median ratio of loan payments to family income for debtors, a common indicator of debt burden, at 18.6 percent, barely rose over the period; in the previous three years, this measure had risen more steeply. But data from the recent three-year period show an increase of 2.5 percentage points in the proportion of debtors with loan payments exceeding 40 percent of their income, a level traditionally considered to be high; the share of families with payment ratios this high was 14.7 percent in 2007.

\section{APPENDIX: \\ SURVEY PROCEDURES AND STATISTICAL MEASURES}

Detailed documentation of the SCF methodology is available elsewhere. ${ }^{67}$ The 2007 data used here are derived from the final internal version of the survey information. Data from this survey, suitably altered to protect the privacy of respondents, along with additional tabulations of data from the surveys beginning with 1989, are expected to be available in February 2009 on the Federal Reserve's website at www. federalreserve.gov/pubs/oss/oss2/2007/scf2007data. html. Links to the data used in this article for earlier periods are available on that site. Results reported in this article for earlier surveys may differ from the results reported in earlier articles because of additional statistical processing, correction of data errors, revisions to the survey weights, conceptual changes in the definitions of variables used in the articles, and adjustments for inflation.

As a part of the general reconciliations required for this article, the survey data were compared with many external estimates, a few of which are mentioned in

67. See Arthur B. Kennickell (2000), "Wealth Measurement in the Survey of Consumer Finances: Methodology and Directions for Future Research" (Washington: Board of Governors of the Federal Reserve System, May); Arthur B. Kennickell (2001), "Modeling Wealth with Multiple Observations of Income: Redesign of the Sample for the 2001 Survey of Consumer Finances" (Washington: Board of Governors of the Federal Reserve System, October), www.federalreserve.gov/ pubs/oss/oss $2 /$ method.html; and references cited in these papers. 
the text. Generally, the survey estimates correspond fairly well to external estimates. One particularly important comparison is between the SCF and the Federal Reserve's flow of funds accounts for the household sector. This comparison suggests that when the definitions of the variables in the two sources can be adjusted to a common conceptual basis, the estimates of totals in the two systems tend to be close. The data series in the SCF and in the flow of funds accounts usually show very similar growth rates. ${ }^{68} \mathrm{In}$ general, the data from the SCF can be compared with those of other surveys only in terms of the medians because of the special design of the SCF sample.

\section{Adjustment for Inflation}

In this article, all dollar amounts from the SCF are adjusted to 2007 dollars using the "current methods" version of the consumer price index (CPI) for all urban consumers. In an ongoing effort to improve accuracy, the Bureau of Labor Statistics has introduced several revisions to its CPI methodology. The current-methods index attempts to extend these changes to earlier years to obtain a series as consistent as possible with current practices in the official CPI. ${ }^{69}$ To adjust assets and liabilities to 2007 dollars and to adjust family income for the preceding calendar year to 2007, the figures given in table A.1 were applied.

A.1.

\begin{tabular}{c|c|c}
\hline Survey year & $\begin{array}{c}\text { Adjustment factor } \\
\text { for assets and debts in } \\
\text { the survey year }\end{array}$ & $\begin{array}{c}\text { Adjustment factor for } \\
\text { income in the calendar year } \\
\text { before the survey year }\end{array}$ \\
\hline $1998 \ldots \ldots \ldots \ldots$ & 1.2732 & 1.2910 \\
$2001 \ldots \ldots \ldots \ldots$ & 1.1696 & 1.2024 \\
$2004 \ldots \ldots \ldots \ldots$ & 1.0983 & 1.1280 \\
\hline
\end{tabular}

\section{Definition of "Family" in the SCF}

The definition of "family" used throughout this article differs from that typically used in other government studies. In the SCF, a household unit is divided into a "primary economic unit" (PEU) - the familyand everyone else in the household. The PEU is intended to be the economically dominant single

68. For details on how these comparisons are structured and the results of comparisons for earlier surveys, see Rochelle L. Antoniewicz (2000), "A Comparison of the Household Sector from the Flow of Funds Accounts and the Survey of Consumer Finances" (Washington: Board of Governors of the Federal Reserve System, October), www.federalreserve.gov/pubs/oss/oss2/method.html.

69. For technical information about the construction of this index, see Kenneth J. Stewart and Stephen B. Reed (1999), "Consumer Price Index Research Series Using Current Methods, 1978-1998," Monthly Labor Review, vol. 122 (June), pp. 29-38. person or couple (whether married or living together as partners) and all other persons in the household who are financially interdependent with that economically dominant person or couple.

This report also designates a head of the PEU, not to convey a judgment about how an individual family is structured but as a means of organizing the data consistently. If a couple is economically dominant in the PEU, the head is the male in a mixed-sex couple or the older person in a same-sex couple. If a single person is economically dominant, that person is designated as the family head in this report.

\section{Percentiles of the Distributions of Income and Net Worth}

Throughout this article, references are made to various percentile groups of the distributions of income or net worth. For a given characteristic, a percentile can be used to define a family's rank relative to other families. For example, the 10th percentile of the distribution of income is the amount of income received by a family for whom just less than 10 percent of families have lower income and 90 percent have higher income. The percentiles of the distributions of income and net worth used to define the income and net worth groups in the tables in the article are given in table A.2.

A.2.

\begin{tabular}{|c|c|c|c|c|}
\hline \multirow{2}{*}{ Item } & \multicolumn{4}{|c|}{ Survey year } \\
\hline & 1998 & 2001 & 2004 & 2007 \\
\hline 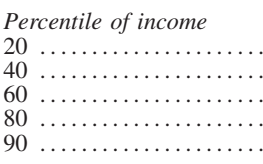 & $\begin{array}{r}17,700 \\
33,600 \\
54,200 \\
86,900 \\
119,600\end{array}$ & $\begin{array}{r}19,700 \\
36,100 \\
60,100 \\
96,200 \\
139,000\end{array}$ & $\begin{array}{r}20,800 \\
37,200 \\
58,900 \\
98,100 \\
142,100\end{array}$ & $\begin{array}{r}20,600 \\
36,500 \\
59,600 \\
98,200 \\
140,900\end{array}$ \\
\hline $\begin{array}{l}\text { Percentile of net worth } \\
25 \\
50 \\
75 \\
75 \ldots \ldots \ldots \ldots \\
90\end{array}$ & $\begin{array}{r}12,700 \\
91,300 \\
265,900 \\
628,300\end{array}$ & $\begin{array}{r}14,900 \\
101,200 \\
335,800 \\
865,700\end{array}$ & $\begin{array}{r}14,600 \\
102,200 \\
360,700 \\
913,300\end{array}$ & $\begin{array}{r}14,100 \\
120,300 \\
372,000 \\
908,200\end{array}$ \\
\hline
\end{tabular}

The groups that are created when a distribution is divided at every 10th percentile are commonly referred to as deciles. Similarly, when a distribution is divided at every 20th (25th) percentile, the groups are known as quintiles (quartiles). Families in the first income decile, for example, are those with income below the 10th percentile.

\section{Racial and Ethnic Identification}

In this article, the race and ethnicity of a family in the SCF are classified according to the self-identification of that family's original respondent to the SCF inter- 
view. The questions underlying the method of classification used in the survey were changed in both 1998 and 2004. Starting in 1998, SCF respondents were allowed to report more than one racial identification; in surveys before then, only one response was recorded. For maximum comparability with earlier data, respondents reporting multiple racial identifications were asked to report their strongest racial identification first.

Beginning with the 2004 survey, the question on racial identification is preceded by a question on whether respondents consider themselves to be Hispanic or Latino in culture or origin; previously, such ethnic identification was captured only to the extent that it was reported as a response to the question on racial identification. The sequence of these two questions in the 2004 SCF is similar to that in the CPS. When families in the March 2004 CPS are classified in the way most compatible with the SCF, the proportion of Hispanic families is 10.5 percent; the 2004 SCF estimate is 11.2 percent. Differences in these proportions are attributable to sampling error and possibly to differences in the wording and context of the questions.

For greater comparability with the earlier SCF data, the data reported in this article ignore the information on ethnic identification available in 2007, but respondents reporting multiple racial identifications in the surveys starting with 1998 are classified as "nonwhite or Hispanic." In the 2007 SCF, 5.4 percent of respondents reported more than one racial identification, up from 2.3 percent in 2004 and 1.5 percent in 2001. Of those who responded affirmatively to the question on Hispanic or Latino identification in 2007, 82.8 percent also reported "Hispanic or Latino" as one of their racial identifications, and 74.5 percent reported it as their primary racial identification. Because the question on Hispanic or Latino ethnicity precedes the one on racial identification in the 2004 and 2007 surveys, the answer to the second of these two questions may have been influenced by the answer to the first. ${ }^{70}$

\section{The Sampling Techniques}

The survey is expected to provide a core set of data on family income, assets, and liabilities. The major

70. For a review of the effects of various approaches to measuring race and ethnicity, see Clyde Tucker, Ruth McKay, Brian Kojetin, Roderick Harrison, Manuel de la Puente, Linda Stinson, and Ed Robinson (1996), "Testing Methods of Collecting Racial and Ethnic Information: Results of the Current Population Survey Supplement on Race and Ethnicity," BLS Statistical Notes 40, CPS Publications (Washington: Bureau of Labor Statistics, June), www.bls.census.gov/ cps/racethn/1995/stat40rp.htm. aspects of the sample design that address this requirement have been constant since 1989. The SCF combines two techniques for random sampling. First, a standard multistage area-probability sample (a geographically based random sample) is selected to provide good coverage of characteristics, such as homeownership, that are broadly distributed in the population.

Second, a supplemental sample is selected to disproportionately include wealthy families, which hold a relatively large share of such thinly held assets as noncorporate businesses and tax-exempt bonds. Called the "list sample," this group is drawn from a list of statistical records derived from tax returns. These records are used under strict rules governing confidentiality, the rights of potential respondents to refuse participation in the survey, and the types of information that can be made available. Persons listed by Forbes magazine as being among the wealthiest 400 people in the United States are excluded from sampling.

Of the 4,422 interviews completed for the 2007 SCF, 2,915 were from the area-probability sample, and 1,507 were from the list sample; for 2004, 3,007 were from the area-probability sample, and 1,515 were from the list sample. The number of families represented in the surveys considered in this article is given by table A.3.

A.3.

\begin{tabular}{c|c}
\hline Year & Number of families represented (millions) \\
\hline $1998 \ldots \ldots \ldots$ & 102.6 \\
$2001 \ldots \ldots \ldots$ & 106.5 \\
$2004 \ldots \ldots \ldots$ & 112.1 \\
$2007 \ldots \ldots \ldots$ & 116.1 \\
\hline
\end{tabular}

\section{The Interviews}

The survey questionnaire has changed in only minor ways since 1989, except in a small number of instances in which the structure was altered to accommodate changes in financial behaviors, in types of financial arrangements available to families, and in regulations covering data collection. In these cases and in all earlier ones, every effort has been made to ensure the maximum degree of comparability of the data over time. Except where noted in the article, the data are highly comparable over time.

The generosity of families in giving their time for interviews has been crucial to the SCF. In the 2007 SCF, the median interview length was about 80 minutes. However, in some particularly complicated cases, the amount of time needed was substantially more than two hours. The role of the interviewers in 
this effort is also critical. Without their dedication and perseverance, the survey would not be possible.

The SCF interviews were conducted largely between the months of May and December in each survey year by NORC, a social science and survey research organization at the University of Chicago. The majority of interviews were obtained in person, although interviewers were allowed to conduct telephone interviews if that was more convenient for the respondent. Each interviewer used a program running on a laptop computer to administer the survey and collect the data.

The use of computer-assisted personal interviewing has the great advantage of enforcing systematic collection of data across all cases. The computer program developed to collect the data for the SCF was tailored to allow the collection of partial information in the form of ranges whenever a respondent either did not know or did not want to reveal an exact dollar figure.

The response rate in the area-probability sample is more than double that in the list sample. In both 2004 and 2007, about 70 percent of households selected for the area-probability sample actually completed interviews. The overall response rate in the list sample was about 30 percent; in the part of the list sample likely containing the wealthiest families, the response rate was only about 10 percent.

\section{Weighting}

To provide a measure of the frequency with which families similar to the sample families could be expected to be found in the population of all families, an analysis weight is computed for each case, accounting both for the systematic properties of the sample design and for differential patterns of nonresponse. The SCF response rates are low by the standards of some other major government surveys, and analysis of the data confirms that the tendency to refuse participation is highly correlated with net worth. However, unlike other surveys, which also almost certainly have differential nonresponse by wealthy households, the SCF has the means to adjust for such nonresponse. A major part of SCF research is devoted to the evaluation of nonresponse and adjustments for nonresponse in the analysis weights of the survey. ${ }^{71}$

71. The weights used in this article are adjusted for differential rates of nonresponse across a number of groups. See Arthur B. Kennickell (1999), "Revisions to the SCF Weighting Methodology: Accounting for Race/Ethnicity and Homeownership" (Washington:

\section{Sources of Error}

Errors may be introduced into survey results at many stages. Sampling error-the variability expected in estimates based on a sample instead of a census-is a particularly important source of error. Such error can be reduced either by increasing the size of a sample or, as is done in the SCF, by designing the sample to reduce important sources of variability. Sampling error can be estimated, and for this article we use replication methods to do so.

Replication methods draw samples, called replicates, from the set of actual respondents in a way that incorporates the important dimensions of the original sample design. In the SCF, weights were computed for all the cases in each of the replicates..$^{72}$ For each statistic for which standard errors are reported in this article, the weighted statistic is estimated using the replicate samples, and a measure of the variability of these estimates is combined with a measure of the variability due to imputation for missing data to yield the standard error.

Other errors include those that interviewers may introduce by failing to follow the survey protocol or misunderstanding a respondent's answers. SCF interviewers are given lengthy, project-specific training to minimize such problems. Respondents may introduce error by interpreting a question in a sense different from that intended by the survey. For the SCF, extensive pretesting of questions and thorough review of the data tend to reduce this source of error.

Nonresponse-either complete nonresponse to the survey or nonresponse to selected items within the survey - may be another important source of error. As noted in more detail above, the SCF uses weighting to adjust for differential nonresponse to the survey. To address missing information on individual questions within the interview, the SCF uses statistical methods to impute missing data; the technique makes multiple estimates of missing data to allow for an estimate of the uncertainty attributable to this type of nonresponse.

\footnotetext{
Board of Governors of the Federal Reserve System, January), www.federalreserve.gov/pubs/oss/oss2/method.html.

72. See Arthur B. Kennickell (2000), "Revisions to the Variance Estimation Procedure for the SCF" (Washington: Board of Governors of the Federal Reserve System, October), www.federalreserve.gov/ pubs/oss/oss2/method.html.
} 


\section{ERRATA}

In the analysis of the SCF reported in the article, privately held businesses do not include businesses that were reported to have a net value of zero; this fact was not made clear in the definition given in footnote 39. In $2007,12.0$ percent of families had a privately held business with a value different from zero; the median and mean values for families having such businesses were $\$ 100,500$ and $\$ 1,071,100$, respectively. If businesses with a value of zero are included in the business definition in 2007, ownership rises to 13.6 percent of families, and the median and mean values fall to $\$ 92,200$ and $\$ 946,300$, respectively. 